\title{
GENERALIZED MAXIMUM FLOW ALGORITHMS
}

\author{
A Dissertation \\ Presented to the Faculty of the Graduate School \\ of Cornell University \\ in Partial Fulfillment of the Requirements for the Degree of \\ Doctor of Philosophy
}

by

Kevin Daniel Wayne

January 1999 
(C) Kevin Daniel Wayne 1999

ALL RIGHTS RESERVED 


\title{
GENERALIZED MAXIMUM FLOW ALGORITHMS
}

\author{
Kevin Daniel Wayne, Ph.D. \\ Cornell University 1999
}

We present several new efficient algorithms for the generalized maximum flow problem. In the traditional maximum flow problem, there is a capacitated network and the goal is to send as much of a single commodity as possible between two distinguished nodes, without exceeding the arc capacity limits. The problem has hundreds of applications including: shipping freight in a transportation network and pumping fluid through a hydraulic network.

In traditional networks, there is an implicit assumption that flow is conserved on every arc. Many practical applications violate this conservation assumption. Freight may be damaged or spoil in transit; fluid may leak or evaporate. In generalized networks, each arc has a positive multiplier associated with it, representing the fraction of flow that remains when it is sent along that arc. The generalized maximum flow problem is identical to the traditional maximum flow problem, except that it can also model networks which "leak" flow. 


\section{Biographical Sketch}

Kevin Wayne was born on October 16, 1971 in Philadelphia, Pennsylvania. After failing a placement exam in seventh grade, Kevin was assigned to a remedial math class, where he has found memories of modeling conic sections with clay. By the end of high school, he was taking advanced calculus classes at the University of Pennsylvania, where he realized there was more to math than playing with clay.

As an undergraduate at Yale University, Kevin stumbled upon a linear programming course, which introduced him to the area of operations research. In 1993, Yale cut the Department of Operations Research, but not before Kevin was graduated magna cum laude with a Bachelor of Science.

In Fall 1993, Kevin began the Ph.D. program at Cornell University in the School of Operations Research and Industrial Engineering. During his first week in Ithaca, he broke his arm playing football, after crashing into a soccer goal post in the endzone. This was a harbinger of things to come. While recovering from his first injury, Kevin quickly rebroke his arm on the basketball court. He also became passionate about several new classes: skiing, cooking, wine tasting, and network flows. The first class led to a broken collarbone, the middle two led to many lavish 
dinner parties, and the last one ultimately led to his dissertation. While writing his dissertation, Kevin broke his right hand diving for a softball. At this point he realized that soccer was his favorite sport, writing a dissertation one-handed is not much fun, and it's better to be left-handed.

In the fall, Kevin will be continuing his tour through the Ivy League. He is very excited to begin teaching at Princeton University in the Department of Computer Science. 
To my loving family. 


\section{Acknowledgments}

First, I would like to express my deepest gratitude to my dissertation supervisor

and mentor Éva Tardos. Without her guidance, encouragement, patience, and inspiration, the research for this dissertation never would have taken place. I am also grateful to David Shmoys who acted as a proxy at both my A-exam and Bexam, and to Jon Kleinberg for serving on my thesis committee.

I am also especially grateful to Professors Jon Lee, Jim Renegar, Sid Resnick, David Shmoys, Éva Tardos, and Mike Todd. These exceptional faculty have taught me so much over the years, and have contributed significantly to my intellectual and professional development.

I am eternally grateful to Mom, Dad, Michele, and Poppop for all of the love and support they have given me over the years. Without their nourishment, I never would have had the chance to succeed.

Also, I am grateful for my extended Gaslight family: Agni, Chris, Dawn, Jim, Jim, John, Mark, Steve, and Vika. I will always have many fond memories of wonderful food, broken doors, jalapeño-eating squirrels, and dead fish. I am also lucky to have such wonderful officemates over the past five years. They have provided 
lasting friendship, enlightenment, encouragement, and entertainment. Thank you Agni, Jim, Kathy, Vardges, and Vika. I am also indebted to Nathan and Michael for their computer wizardry and $\mathrm{LT}_{\mathrm{E}} \mathrm{X} 2_{\varepsilon}$ support.

I wish to thank the Defense Advanced Research Projects Agency for supporting my studies via a National Defense Science and Engineering Graduate Fellowship. I am also indebted to the Office of Naval Research for supporting my research through grant AASERT N00014-97-1-0681.

Last, but certainly not least, I would like to thank my orthopedic. 


\section{Table of Contents}

1 Introduction 1

1.1 Network Flows . . . . . . . . . . . . . . . . . . . . . . . . . . 1

1.2 Generalized Maximum Flow Problem . . . . . . . . . . . . . . . 2

1.3 Applications . . . . . . . . . . . . . . . . . 3

1.4 Measuring Efficiency of Algorithms . . . . . . . . . . . . . 6

1.5 Approximation Algorithms . . . . . . . . . . . . . . . . 7

1.6 The Combinatorial Approach . . . . . . . . . . . . . 8

1.7 Overview of Dissertation . . . . . . . . . . . . . . . 9

2 Preliminaries $\quad 11$

2.1 Basic Definitions . . . . . . . . . . . . . . . . . . 11

2.2 Some Traditional Network Flow Problems . . . . . . . . . . . . . . 14

2.2.1 Shortest Path Problem . . . . . . . . . . . . . . . . . . 14

2.2.2 Minimum Mean Cycle Problem . . . . . . . . . . . . . . 15

2.2.3 Maximum Flow Problem . . . . . . . . . . . . . . . 15

2.2.4 Minimum Cut Problem . . . . . . . . . . . . . . . 17

2.2.5 Minimum Cost Flow Problem . . . . . . . . . . . . . . . 18

2.3 Generalized Maximum Flow Problem . . . . . . . . . . . . . . . . 20

2.3.1 Generalized Residual Network . . . . . . . . . . . . . . . . . 22

2.3.2 Relabeled Network . . . . . . . . . . . . . . . . . 24

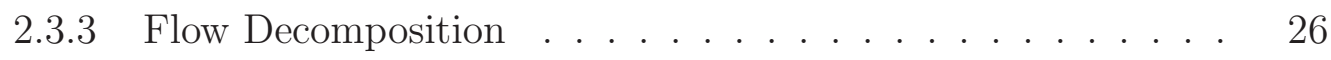

2.3.4 Optimality Conditions . . . . . . . . . . . . . 30

2.4 Canceling All Flow-Generating Cycles . . . . . . . . . . . . . . . . . . . 32

2.5 Nearly-Optimal Flows . . . . . . . . . . . . . . . 33

3 Generalized Maximum Flow Literature $\quad 37$

3.1 Combinatorial Methods . . . . . . . . . . . . . . 38

3.2 Linear Programming Methods . . . . . . . . . . . . . . . . . . . 41

3.3 Best Complexity Bounds . . . . . . . . . . . . . . . 42 
4 Gain-Scaling 44

4.1 Rounding Down the Gains . . . . . . . . . . . . . . . . . . 45

4.2 Error-Scaling . . . . . . . . . . . . . . . . . . . 47

5 Augmenting Path Algorithms 49

5.1 Augmenting Path Algorithm . . . . . . . . . . . . . . . 49

5.2 Primal-Dual Algorithm . . . . . . . . . . . . . . . . . . 50

5.3 Rounded Primal-Dual . . . . . . . . . . . . . . . . . 53

5.4 Recursive Rounded Primal-Dual . . . . . . . . . . . . . . . . 54

6 Push-Relabel Method $\quad 58$

6.1 Push-Relabel Method for Min Cost Flow . . . . . . . . . . . . . . . 59

6.2 Push-Relabel for Generalized Flows . . . . . . . . . . . . . . . 62

6.3 Recursive Rounded Push-Relabel . . . . . . . . . . . . . . . . 69

6.4 Issues for a Practical Implementation . . . . . . . . . . . . . . 70

7 Fat-Path $\quad 71$

7.1 Most-Improving Augmenting Path . . . . . . . . . . . . . . 71

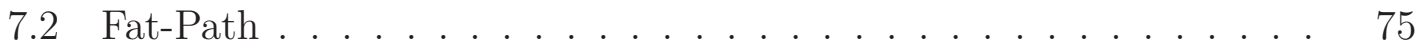

7.3 Fat Augmentations . . . . . . . . . . . . . . . . . . . 79

7.4 Radzik's Fat-Path Variant . . . . . . . . . . . . . . . . . 86

7.5 Rounded Fat-Path . . . . . . . . . . . . . . . . 86

7.5.1 Rounded Fat-Path . . . . . . . . . . . . . . 87

7.5.2 Recursive Rounded Fat-Path . . . . . . . . . . . . . . . 88

8 Canceling Flow-Generating Cycles 94

8.1 Cancel Cycles . . . . . . . . . . . . . . . . . . 95

8.2 Our Cancel Cycles Variant . . . . . . . . . . . . . . . . . . 103

$\begin{array}{ll}\text { Bibliography } & 111\end{array}$ 


\section{List of Figures}

1.1 Some Examples of Networks . . . . . . . . . . . . . . . . . . 2

1.2 Gain Factors . . . . . . . . . . . . . . . . . . . . 3

1.3 Currency Conversion Problem . . . . . . . . . . . . . 5

1.4 Machine Scheduling Problem . . . . . . . . . . . . . . . 6

2.1 Rounding to an Optimal Flow _. . . . . . . . . . . . 34

4.1 Flow Interpretation $\ldots \ldots \ldots \ldots \ldots$

$5.1 \quad$ Algorithm Primal-Dual _. . . . . . . . . . . . . . . . 51

5.2 Algorithm Rounded Primal-Dual . . . . . . . . . . . . . . . . 53

5.3 Algorithm Recursive Rounded Primal-Dual . . . . . . . . . . . . 55

6.1 Algorithm Push-Relabel . . . . . . . . . . . . . . . . . . . . 60

6.2 Algorithm Rounded Push-Relabel . . . . . . . . . . . . . . . 63

6.3 Algorithm Recursive Rounded Push-Relabel . . . . . . . . . . . . 70

7.1 Algorithm Most-Improving Augmenting Path . . . . . . . . . . 72

7.2 Subroutine Finding a Most-Improving Augmenting Path . . . . . . 74

7.3 Algorithm Fat-Path . . . . . . . . . . . . . . . . . 77

7.4 Subroutine Fat Augmentations . . . . . . . . . . . . . 82

7.5 Algorithm Rounded Fat-Path . . . . . . . . . . . . . . . 87

7.6 Algorithm Recursive Rounded Fat-Path . . . . . . . . . . . 90

8.1 Subroutine Cancel Cycles . . . . . . . . . . . . . . . . . . . 98

8.2 Subroutine Cancel Cycles2 _. . . . . . . . . . . . . . . . 104 


\section{Chapter 1}

\section{Introduction}

\section{$1.1 \quad$ Network Flows}

We encounter many different types of networks in our everyday lives, including electrical, telephone, cable, highway, rail, manufacturing, and, computer networks. Networks consists of special points called nodes and links connecting pairs of nodes called arcs. Some examples of networks are listed in Figure 1.1, which is taken from [1]. In all of these networks, we wish to send some commodity, which we generically call flow, from one node to another, and do so as efficiently as possible, subject to certain constraints. Network flow theory is the study of designing computationally efficient algorithms to solve such problems. 


\begin{tabular}{|l|l|l|l|}
\hline Network & Nodes & Arcs & Flow \\
\hline communication & $\begin{array}{l}\text { telephone exchanges, } \\
\text { computers, satellites }\end{array}$ & $\begin{array}{l}\text { cables, fiber optics, } \\
\text { microwave relay } \\
\text { links }\end{array}$ & $\begin{array}{l}\text { voice messages, } \\
\text { video, data }\end{array}$ \\
\hline hydraulic & $\begin{array}{l}\text { reservoirs, lakes, } \\
\text { pumping stations }\end{array}$ & pipelines & $\begin{array}{l}\text { hydraulic fluid, } \\
\text { water, gas, oil }\end{array}$ \\
\hline financial & currencies, stocks & transactions & money \\
\hline transportation & $\begin{array}{l}\text { airports, rail yards, } \\
\text { intersections }\end{array}$ & $\begin{array}{l}\text { highways, railbeds, } \\
\text { airline routes }\end{array}$ & $\begin{array}{l}\text { freight, vehicles, } \\
\text { passengers }\end{array}$ \\
\hline
\end{tabular}

Figure 1.1: Some Examples of Networks

\subsection{Generalized Maximum Flow Problem}

In this dissertation, we consider a network flow problem called the generalized maximum flow problem. First, we describe the traditional maximum flow problem. This problem was first studied by Dantzig [11] and Ford and Fulkerson [15] in the 1950's. The problem is simple to state and is defined formally in Section 2.2.3: given capacity limits on the arcs, the goal is to send as much flow as possible from one distinguished node called the source to another called the sink. For example, a power company may wish to maximize the amount of natural gas sent between a pair of cities through its network of pipelines. Each pipeline in the network has a limited capacity.

The generalized maximum flow problem is a natural generalization of the traditional maximum flow problem. It was first investigated by Dantzig [12] and Jewell [33] in the 1960's. In traditional networks, there is an implicit assumption that flow is conserved on every arc. This assumption may be violated if natural gas 
leaks as it is pumped through a pipeline. ${ }^{1}$ The generalized maximum flow problem generalizes the traditional maximum flow problem by allowing flow to "leak" as it is sent through the network. As before, each $\operatorname{arc}(v, w)$ has a capacity $u(v, w)$ that limits the amount of flow sent into that arc. Additionally, each arc $(v, w)$ has a positive multiplier $\gamma(v, w)$, called a gain factor, associated with it. For each unit of flow entering the arc, $\gamma(v, w)$ units exit. As the example in Figure 1.2 illustrates, if 80 units of flow are sent into an arc $(v, w)$ with gain factor $3 / 4$, then 60 units reach node $w$; if these 60 units are then sent through an $\operatorname{arc}(w, x)$ with gain factor $1 / 2$, then 30 units arrive at $x$.

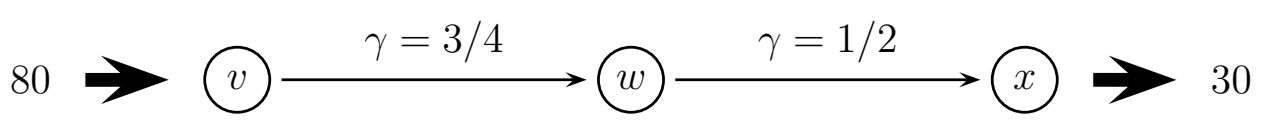

Figure 1.2: Gain Factors

\subsection{Applications}

In traditional networks, there is an implicit assumption that flow is conserved on every arc. Many practical applications violate this conservation assumption. The gain factors can represent physical transformations of one commodity into a lesser or greater amount of the same commodity. Some examples include: spoilage, theft, evaporation, taxes, seepage, deterioration, interest, or breeding. The gain factors can also model the transformation of one commodity into a different commodity.

\footnotetext{
${ }^{1}$ We hope that gas does not actually leak from the pipeline. However, gas in the pipeline is used to drive the pipeline pumps; effectively, gas leaks as it is shipped through the pipeline.
} 
Some examples include: converting raw materials into finished goods, currency conversion, and machine scheduling. We explain the latter two examples next.

\section{Currency conversion}

We use the currency conversion problem as an illustrative example of the types of problems that can be modeled using generalized flows. Later, we will use this problem to gain intuition. In the currency conversion problem, the goal is to take advantage of discrepancies in currency conversion rates. Given a certain amount of one currency, say 1000 U.S. dollars, the goal is to convert it into the maximum amount of another currency, say French Francs, through a sequence of currency conversions. We assume that limited amounts of currency can be traded without affecting the exchange rates.

We model the currency conversion problem as a generalized maximum flow problem in Figure 1.3. Each node represents a currency, and each arc represents a possible transaction that converts one currency into another. The source node is dollars and the sink node is Francs. The gain factor of the arc between two currencies, say directly from dollars to Francs, is the exchange rate. The capacity of that arc is the maximum number of the first currency that we can convert into the second currency. In the example, we can directly convert up to 800 dollars into Francs at the exchange rate of five Francs per dollar. Note that in this example, it is more efficient to convert indirectly from dollars to Deutsch Marks to Francs; using this sequence of conversions we get an effective exchange rate of six Francs per dollar. 


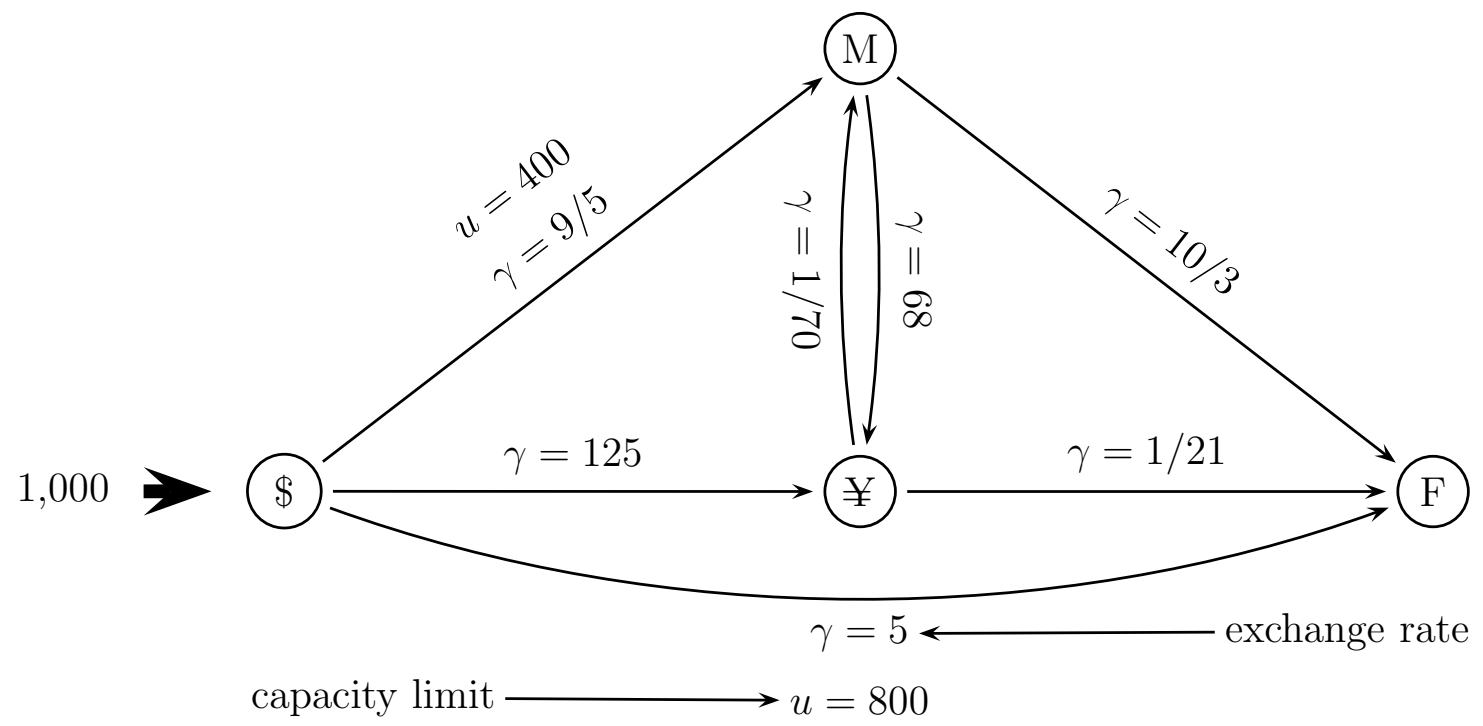

Figure 1.3: Currency Conversion Problem

\section{Scheduling unrelated parallel machines}

As a second example, we consider the problem of scheduling $N$ jobs to run on $M$ unrelated machines. The goal is to schedule all of the jobs by a prespecified time $T$. Each job must be assigned to exactly one machine. Each machine can process any of the jobs, but at most one job at a time. Machine $i$ requires a pre-specified amount of time $p_{i j}$ to process job $j$.

The problem can be formulated as an integer program using assignment variables to indicate whether job $j$ is processed on machine $i$. The natural linear programming relaxation is formulated below as a generalized maximum flow problem. The optimal linear programming solution can be appropriately rounded [41] to produce an approximately optimal schedule for the original problem. 
We model the machine scheduling problem as a generalized maximum flow problem in Figure 1.3. We have a node for each machine $i$ and a node for each job $j$. Each machine has a supply of $T$ units of time. Each job has a demand of one. There is an uncapacitated arc from machine $i$ to job $j$ with gain factor $1 / p_{i j}$, representing the rate at which machine $i$ processes job $j$. The flow on arc $(i, j)$ represents the amount of time machine $i$ spends processing job $j$.

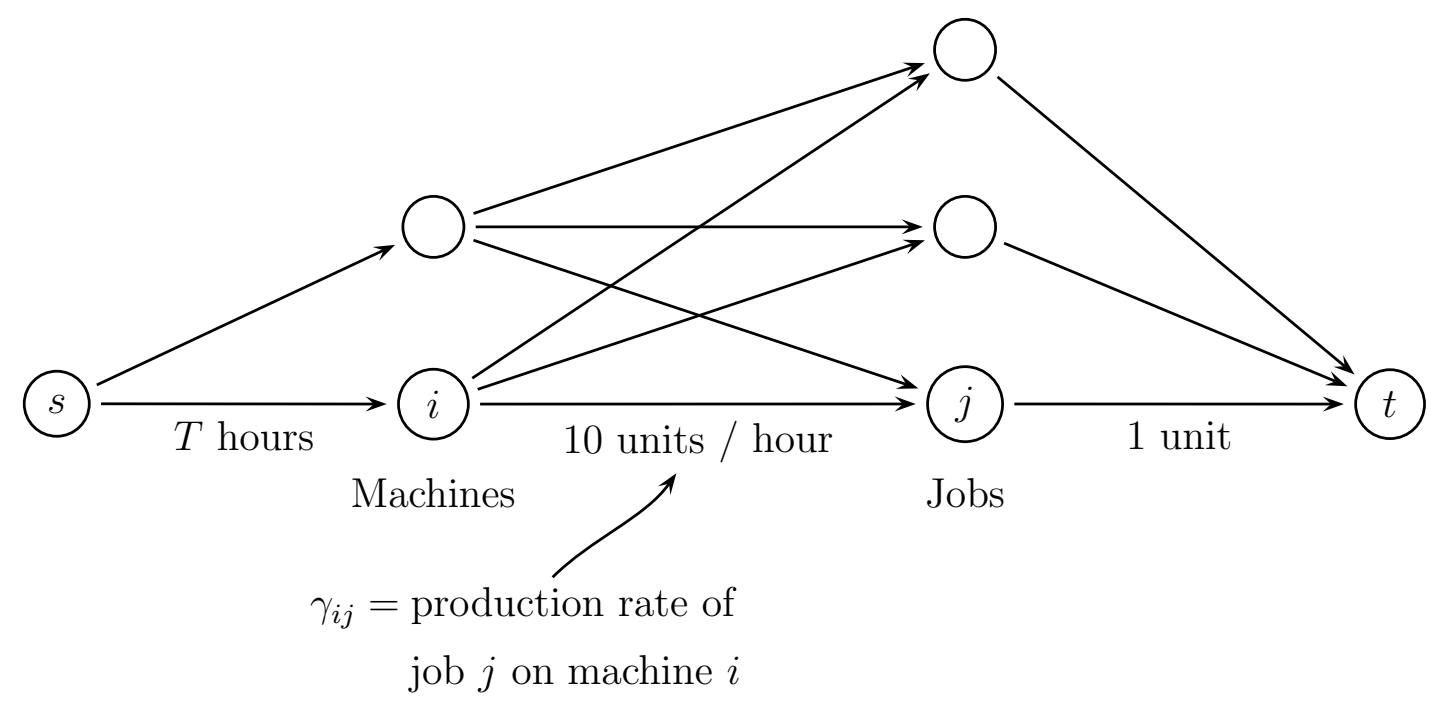

Figure 1.4: Machine Scheduling Problem

\subsection{Measuring Efficiency of Algorithms}

Ideally, we would like to measure the efficiency of an algorithm based on its ability to perform well on practical problem. This notion is vague and lacks theoretical grounding. We measure an algorithm based on its worst-case complexity, i.e., the maximum number of machine operations that the algorithm requires on any problem 
instance of a given size. For network flow problems, the size depends on the number of $\operatorname{arcs} m$, the number of nodes $n$, and the biggest integer $B$ used to represent capacities, gain factors, and costs. A network flow algorithm is called a polynomialtime algorithm if its worst-case complexity is bounded by a polynomial function of $m, n$, and $\log _{2} B$. We use $\log _{2} B$ because it represents the number of bits needed to store the integer $B$ on a binary computer.

Comparing the performance of algorithms based on their worst-case complexity has gained widespread acceptance over the past three decades. For a given problem, the goal is to design a polynomial-time algorithm with the smallest worst-case complexity. There are many reasons to justify such a goal. First, this provides a mathematical framework in which we can compare different algorithms. Second, there is strong computational evidence suggesting a high correlation between an algorithm's worst-case complexity and its practical performance. Finally, the study of polynomial-time algorithms has led to dramatic advances and innovations in the design of new practical algorithms for a wide variety of problems.

\subsection{Approximation Algorithms}

For many practical optimization applications, we are often satisfied with solutions that may not be optimal, but are guaranteed to be "close" to optimal. For example, if the input data to the problem is only known to a certain level of precision, then it is often acceptable to only produce a solution of the same level of precision. A second important reason is that we can often tradeoff solution quality for computa- 
tional speed; in many applications we can find a provably high quality solution in substantially less time than it would take to find an optimal solution.

A $\xi$-approximation algorithm for an optimization problem is a polynomial-time algorithm that is guaranteed to produce a solution that is within a factor of $(1-\xi)$ of the optimum. For example, if $\xi=0.01$ then a $\xi$-approximation algorithm for the maximum flow problem produces a flow that has value at least $99 \%$ as large as the optimum, i.e., it is within $1 \%$ of the best possible.

We design both exact and approximation algorithms for the generalized maximum flow problem. We present a family of $\xi$-approximation algorithms for every $\xi>0$. This means that we can find nearly optimal solutions to any prescribed level of precision. For example, when $\xi=0.01$ our approximation algorithms are faster than our exact algorithms by roughly a factor of $m$, where $m$ is the number of arcs in the underlying network.

\subsection{The Combinatorial Approach}

Since the generalized flow problem can be formulated as a linear program, it can be solved by general purpose linear programming methods including simplex, ellipsoid, and interior point methods. These continuous optimization methods are grounded in linear algebra.

The problem can also be solved by combinatorial methods. Combinatorial methods exploit the discrete structure of the underlying network, often using graph search, shortest path, maximum flow, and minimum cost flow computations as sub- 
routines. These methods have led to superior algorithms for many traditional network flow problems including the shortest path, maximum flow, minimum cost flow, and matching problems. More recently, combinatorial methods have been used to develop fast approximation algorithms for packing and covering linear programming problems, including multicommodity flow.

We believe that the best generalized flow algorithms will come from techniques that exploit the combinatorial structure of the underlying network. This dissertation takes an important step in this direction.

\subsection{Overview of Dissertation}

In Chapter 2, we review some basic facts about network flows and generalized flows that we will use in our algorithms.

In Chapter 3, we review the literature for the generalized maximum flow problem.

In Chapter 4, we introduce a gain-scaling methodology for generalized network flow problems. Scaling is a powerful technique for deriving polynomial-time algorithms for a wide variety of combinatorial optimization problems. Almost all of the best traditional network flow algorithms use some form of capacity or cost scaling. Prior to this thesis, bit-scaling techniques did not appear to apply to generalized flow problems, in part because there is no integrality theorem. Our gain-scaling technique provides the basic tools necessary to design several new efficient generalized maximum flow algorithms. 
The primal-dual algorithm is one of the simplest algorithms for the problems, but requires exponential time. In Chapter 5, we present a polynomial-time variant. It is the simplest and cleanest polynomial-time approximation algorithm for the problem.

In Chapter 6, we adapt the push-relabel method of [22] to generalized flows. The push-relabel method is currently the most practical algorithm for the traditional maximum flow problem. Our algorithm is the first polynomial-time push-relabel algorithm for generalized flows. We believe that our push-relabel algorithm will be quite practical for computing approximate flows.

In Chapter 7, we design a new variant of the Fat-Path capacity-scaling algorithm of [20]. Our variant matches the best known complexity for the problem, and it is much simpler than the variant in [49].

In Chapter 8, we discuss a strongly-polynomial variant of a procedure of [20] which "cancels all flow-generating cycles." This is used by many of our algorithm to reroute flow from their current paths to more efficient paths. 


\section{Chapter 2}

\section{Preliminaries}

In this chapter we review several fundamental network flow problems. We formally define the generalized maximum flow problem and review some basic facts that we use in the design and analysis of our algorithms.

\subsection{Basic Definitions}

All of the problems we consider are defined on a directed graph $(V, E)$ where $V$ is an $n$-set of nodes and $E$ is an $m$-set of directed arcs. For notational convenience, we assume that the graph has no parallel arcs; this allows us to uniquely specify an arc by its endpoints. Our algorithms easily extend to allow for parallel arcs, and the complexity bounds we present remain valid. We consider only simple directed paths and cycles. 
Lengths. The shortest path and minimum mean cycle problems use a length function $l: E \rightarrow \Re$. The length $l(v, w)$ is the distance from node $v$ to node $w$. We denote the length of a cycle (path) $\Gamma$ by $l(\Gamma)=\sum_{e \in \Gamma} l(e)$.

Costs. The minimum cost flow problem uses a cost function $c: E \rightarrow \Re$. The cost $c(v, w)$ is the unit shipping cost for arc $(v, w)$. We denote the cost of a cycle $\Gamma$ by $c(\Gamma)=\sum_{e \in \Gamma} c(e)$.

Capacities. The maximum flow, minimum cost flow, and generalized maximum flow problem use a capacity function $u: E \rightarrow \Re_{\geq 0}$. The capacity $u(v, w)$ limits the amount of flow we are permitted to send into arc $(v, w)$.

Symmetry. For the maximum flow and minimum cost flow problems, we assume the input network is symmetric, i.e., if $(v, w) \in E$ then $(w, v) \in E$ also. This is without loss of generality, since we could always add the opposite arc and assign it zero capacity. Without loss of generality, we also assume the costs are antisymmetric, i.e., $c(v, w)=-c(w, v)$ for every $\operatorname{arc}(v, w) \in E$. The reason for these assumption will become clear in the next paragraph.

Flows. A pseudoflow $f: E \rightarrow \Re$ is a function that satisfies the capacity constraints:

$$
\forall(v, w) \in E: \quad f(v, w) \leq u(v, w)
$$

and the antisymmetry constraints:

$$
\forall(v, w) \in E: \quad f(v, w)=-f(w, v) .
$$


To gain intuition, it is useful to think of only the nonnegative components of a pseudoflow. The negative flows are introduced for notational convenience. Note that we do not need to distinguish between upper and lower capacity limits.

For example, a flow of 17 units sent along $\operatorname{arc}(v, w)$ is also viewed as a flow of -17 units sent along the reverse arc $(w, v)$. The cost of sending one unit of flow along $(v, w)$ is $c(v, w)$; sending one unit of flow along the opposite $\operatorname{arc}(w, v)$ has cost $-c(v, w)$, and is equivalent to decreasing the flow on $\operatorname{arc}(v, w)$ by one unit. Now, to see how lower bounds are implicitly modeled, suppose $\operatorname{arc}(w, v)$ has zero capacity. This implies that variable $f(v, w)$ is nonnegative: the capacity constraint for $\operatorname{arc}(w, v)$ is $f(w, v) \leq 0$, so then the antisymmetry constraint implies $f(v, w)=-f(w, v) \geq 0$.

Residual Networks. With respect to a pseudoflow $f$ in network $G$, the residual capacity function $u_{f}: E \rightarrow \Re$ is defined by $u_{f}(v, w)=u(v, w)-f(v, w)$. The residual network is $G_{f}=\left(V, E, u_{f}\right)$. Note that the residual network may include arcs with zero residual capacity, and still satisfies the symmetry assumption.

For example, if $u(v, w)=20, u(w, v)=0$, and $f(v, w)=-f(w, v)=17$, then $\operatorname{arc}(v, w)$ has $20-(-17)=3$ units of residual capacity, and $\operatorname{arc}(w, v)$ has $0-(-17)$ $=17$ units of residual capacity.

We define $E_{f}=\left\{(v, w) \in E: u_{f}(v, w)>0\right\}$ to be the set of all arcs in $G_{f}$ with positive residual capacity. A residual arc is an arc with positive capacity. A residual path (cycle) is a path (cycle) consisting entirely of residual arcs. 


\subsection{Some Traditional Network Flow Problems}

In this section we formally define the shortest path, minimum mean cycle, maximum flow, minimum cut, and minimum cost flow problems. We also state the best known complexity bounds. We will use these as subroutines in our generalized flow algorithms.

\subsubsection{Shortest Path Problem}

In the shortest path problem, the goal is to find a simple path between two nodes, so as to minimize the total length. An instance of the shortest path problem is a network $G=(V, E, s, l)$, where $s \in V$ is a distinguished node called the source, and $l$ is a length function. The problem is NP-hard if negative length cycles are allowed. In networks with no negative length cycles, there are a number of polynomialtime algorithms for the problem, e.g. Bellman-Ford. There are faster specialized algorithms for networks with nonnegative arc lengths, e.g., Dijkstra.

We let $\operatorname{SP}(m, n)$ denote the complexity of solving a shortest path problem in a network with $m$ arcs, and $n$ nodes, and nonnegative lengths. Currently, the best known bound for $\operatorname{SP}(m, n)$ is $\mathcal{O}(m+n \log n)$ due to [17]. Recently, Thorup [53] developed a linear time algorithm for the problem; his algorithm performs bit manipulations on the input numbers. We let $\mathrm{SP}(m, n, C)$ be the complexity assuming the lengths are integers between 0 and $C$. Currently, the best known bounds for $\mathrm{SP}(m, n, C)$ are $\mathcal{O}(m \log \log C)$ and $\mathcal{O}(m+n \sqrt{\log C})$ due to [34], and [2], respectively. 
If negative length arcs are allowed (but no negative length cycles), then the best strongly polynomial complexity bound is $\mathcal{O}(m n)$ due to Bellman [5] and Ford [16]. The best weakly polynomial bound is $\mathcal{O}(m \sqrt{n} \log C)$ due to [].

\subsubsection{Minimum Mean Cycle Problem}

In the minimum mean cycle problem, the goal is to find a cycle whose ratio of length to number of arcs is minimum. That is, we want to find a cycle $\Gamma$ that minimizes $l(\Gamma) /|\Gamma|$. An instance of the minimum mean cost cycle problem is a network $G=(V, E, l)$, where $l$ is a length function. Although it is NP-hard to find a cycle of minimum length, it is possible to find a minimum mean cycle in polynomial-time. Virtually all known algorithms are based upon a shortest path computation in a network where negative length arcs are allowed.

We let $\operatorname{MMC}(m, n)$ denote the complexity of finding a minimum mean cost cycle in a network with $m$ arcs, $n$ nodes, and arbitrary costs. Currently, the best known bound for $\operatorname{MMC}(m, n)$ is $\mathcal{O}(m n)$ due to $\operatorname{Karp}$ [38]. We let $\operatorname{MMC}(m, n, C)$ denote the complexity assuming the lengths are integers between $-C$ and $C$. Currently, the best known bound for $\operatorname{MMC}(m, n, C)$ is $\mathcal{O}(m \sqrt{n} \log (n C))$ due to Orlin and Ahuja [46].

\subsubsection{Maximum Flow Problem}

In the maximum flow problem, the goal is to send as much flow as possible between two nodes, subject to arc capacity limits. An instance of the maximum flow problem 
is a network $G=(V, E, s, t, u)$, where $s \in V$ is a distinguished node called the source, $t \in V$ is a distinguished node called the sink, and $u$ is a capacity function. A flow is a pseudoflow that satisfies and the flow conservation constraints:

$$
\forall v \in V-\{s, t\}: \quad \sum_{w \in V:(v, w) \in E} f(v, w)=0 .
$$

This says that for all nodes except the source and sink, the net flow leaving that node is zero. We do not have to distinguish between flow entering and leaving node $v$ because of the antisymmetry constraints. The value of a flow $f$ is the net flow into the sink:

$$
|f|=\sum_{v \in V:(v, t) \in E} f(v, t) .
$$

The objective is to find a flow of maximum value.

An augmenting path is a residual $s$-t path. Clearly if there exists an augmenting path in $G_{f}$, then we can improve $f$ by sending flow along this path. Ford and Fulkerson [15] showed that the converse is also true.

Theorem 2.2.1. A flow $f$ is a maximum flow if and only if $G_{f}$ has no augmenting paths.

This theorem motivates the augmenting path algorithm of Ford and Fulkerson's [15], which repeatedly sends flow along augmenting paths, until no such paths remain. If the original capacities are integral, then the algorithm always augments integral amounts of flow. The following integrality theorem is immediate.

Theorem 2.2.2. If all of the arc capacities are integral, then there exists an integer maximum flow. 
We let $\operatorname{MF}(m, n)$ denote the worst-case complexity of finding a maximum flow in a network with $m$ arcs, $n$ nodes, and arbitrary positive capacities. Currently, the best known bounds on $\operatorname{MF}(m, n)$ are $\mathcal{O}\left(m n \log \left(n^{2} / m\right)\right), \mathcal{O}\left(m n \log _{m / n \log n} n\right)$, and $\mathcal{O}\left(m n \log _{m / n} n+n^{2} \log ^{2+\epsilon} n\right)$ for any constant $\epsilon>0$, due to [22], [39], and [47], respectively. We let $\operatorname{MF}(m, n, U)$ denote the complexity assuming the capacities are integers between 0 and $U$. Currently, the best known bounds for $\operatorname{MF}(m, n, U)$ are $\mathcal{O}\left(m n \log (n \sqrt{\log U} /(m+2))\right.$ and $\mathcal{O}\left(\min \left\{n^{2 / 3}, \sqrt{m}\right\} m \log \left(n^{2} / m\right) \log U\right)$ due to [3], and [21], respectively.

\subsubsection{Minimum Cut Problem}

The $s$ - $t$ minimum cut problem is intimately related to the maximum flow problem. The input is the same as for the maximum flow problem. The goal is to find a partition of the nodes that separates the source and sink, so that the total capacity of arcs going from the source side to the sink side is minimum. Formally, we define an $s$ - $t$ cut $[S, T]$ to be a partition of the nodes $V=S \cup T$ so that $s \in S$ and $t \in T$. The capacity of a cut is defined to be the sum of the capacities of "forward" arcs in the cut:

$$
u[S, T]=\sum_{v \in S, w \in T} u(v, w) .
$$

The goal is to find an $s-t$ cut of minimum capacity.

It is easy to see that the value of any flow is less than or equal to the capacity of any $s-t$ cut. Any flow sent from $s$ to $t$ must pass through every $s-t$ cut, since the cut disconnects $s$ from $t$. Since flow is conserved, the value of the flow is limited by the 
capacity of the cut. A cornerstone result of network flows is the much celebrated max-flow min-cut theorem of Ford and Fulkerson [15]. It captures the fundamental duality between the maximum flow and minimum cut problems.

Theorem 2.2.3. The maximum value of any flow from the source $s$ to the sink $t$ in a capacitated network is equal to the minimum capacity among all s-t cuts.

Proof. By our previous observation, it is sufficient to show that the capacity of some $s-t$ cut equals the value of some flow. Let $f$ be a maximum flow. Choose $S$ to be the set of nodes reachable from the source using only residual arcs in $G_{f}$, and let $T=V \backslash S$. We show that $[S, T]$ is an $s$ - $t$ cut of capacity $|f|$. Clearly $s \in S$ and $t \in T$. By the definition of $S$, flow $f$ saturates every "forward" arc in the cut, and does not send flow along any "backward" arcs in the cut. Thus, the net flow crossing the cut is $u[S, T]$. By flow conservation, the net flow sent across any $s-t$ cut is equal to the value of the flow; thus $u[S, T]=|f|$.

\subsubsection{Minimum Cost Flow Problem}

In the minimum cost flow problem, the goal is to send flow from supply nodes to demand nodes as cheaply as possibly, subject to arc capacity constraints. An instance of the minimum cost flow problem is a network $G=(V, E, b, u, c)$, where $b: V \rightarrow \Re$ is a supply function, $u$ is a capacity function, and $c$ is a cost function. We say node $v \in V$ has supply if $b(v)>0$ and demand if $b(v)<0$. We assume that the total supply equals the total demand, i.e. $\sum_{v \in V} b(v)=0$; otherwise the problem is 
infeasible. A flow is a pseudoflow that satisfies the mass balance constraints:

$$
\forall v \in V: \quad \sum_{w \in V:(v, w) \in E} f(v, w)=b(v) .
$$

Let $f$ be a flow. If there exists a negative cost residual cycle in $G_{f}$, then we can improve $f$ by sending flow around the cycle. Busacker and Saaty [8] showed that the converse is also true.

Theorem 2.2.4. A flow $f$ is a minimum cost flow if and only if $G_{f}$ contains no negative cost residual cycles.

Now, we describe an alternate set of optimality conditions. We refer to a function $\pi: V \rightarrow \Re$ as a set of node potentials. The reduced cost of an $\operatorname{arc}(v, w) \in E$ with respect to node potentials $\pi$ is defined to be:

$$
c_{\pi}(v, w)=c(v, w)-\pi(v)+\pi(w) .
$$

Intuitively, we can view $-\pi(v)$ as the market price for buying or selling one unit of flow at node $v$. The reduced cost is then the cost of buying one unit at node $v$, shipping it to node $w$, and selling it at node $w$.

The complementary slackness optimality conditions express the negative cost cycle optimality conditions in terms of reduced costs. It says the a flow is optimal if and only if there are prices $\pi$ so that there is no incentive to buy flow, ship it, and then sell it.

Theorem 2.2.5. A flow $f$ is a minimum cost flow if and only if there exists a set of node potentials $\pi$ such that:

$$
\forall(v, w) \in E_{f}: \quad c_{\pi}(v, w) \geq 0 .
$$


Proof. Let $f$ be a flow and let $\pi$ be potentials that satisfy (2.3). All residual arcs have nonnegative cost. Then, there are no negative cost residual cycles in $G_{f}$. The cost of a cycle is equal to the reduced cost of the cycle; hence flow $f$ is optimal by Theorem 2.2.4.

Now suppose $f$ is a minimum cost flow. Then by Theorem 2.2.4, there are no negative cost residual cycles in $G_{f}$. Let $\pi(v)$ be the shortest path from node $v$ to some designated node $t$ in the graph $\left(V, E_{g}\right)$ using lengths $c$. The shortest path distances are well-defined and the shortest path optimality conditions imply that $\pi$ satisfies (2.3).

\subsection{Generalized Maximum Flow Problem}

In this section, we formally define the generalized maximum flow problem. We define the residual and the relabeled networks. These networks will be useful in the design of our algorithms. We also characterize the optimality conditions for the problem.

Gains. The generalized maximum flow problem uses a gain function $\gamma: E \rightarrow \Re_{>0}$. For each unit of flow that enters arc $(v, w)$ at node $v$, only $\gamma(v, w)$ units arrive at node w. A lossy arc is an arc with gain factor at most one. Without loss of generality, we assume that the gain function is symmetric, i.e., $\gamma(v, w)=1 / \gamma(w, v)$. If this assumption is not satisfied, we can add the symmetric arc and give it a capacity of zero. We will see that this is a natural assumption in the next paragraph. 
Generalized pseudoflow. A generalized pseudoflow is a function $g: E \rightarrow \Re$ that satisfies the capacity constraints (2.1) and the and generalized antisymmetry constraints:

$$
\forall(v, w) \in E: \quad g(v, w)=-\gamma(w, v) g(w, v)
$$

To gain intuition, it is useful to think of only the positive components of a pseudoflow. As before, the negative flows are introduced for notational convenience and we do not need to distinguish between upper and lower capacity limits.

If we send 200 units of flow into an $\operatorname{arc}(v, w)$ with a gain factor of $1 / 5$, then this would produce 40 units of flow at node $w$. This is also viewed as sending -40 units of flow along arc $(w, v)$, which has a gain factor of 5 .

The problem. In the generalized maximum flow problem, the goal is to send as much flow as possible between two nodes, subject to arc capacity constraints. Also, flow "leaks" as it is sent through the network.

Since some of our algorithms are recursive, it is convenient to solve a seemingly more general version of the problem which allows multiple sources. An instance of the generalized maximum flow problem is a generalized network $G=$ $(V, E, t, u, \gamma, e)$, where $t \in V$ is a distinguished node called the $\sin k, u$ is a capacity function, $\gamma$ is a gain function, and $e: V \rightarrow \Re_{\geq 0}$ is an initial excess function.

The residual excess of a generalized pseudoflow $g$ at node $v$ is defined by:

$$
e_{g}(v)=e(v)-\sum_{(v, w) \in E} g(v, w) .
$$


It is the initial excess minus the net flow leaving $v$. If $e_{g}(v)$ is positive (negative) we say that $g$ has residual excess (deficit) at node $v$. A generalized flow is a generalized pseudoflow that has no residual deficits, but it is allowed to have residual excesses. A proper generalized flow is a flow which does not generate any additional residual excesses, except possibly at the sink. We will show in Corollary 2.3.6 that a flow can be efficiently converted into a proper flow that generates the same amount of residual excess at the sink. For a flow $g$ we denote its value $|g|=e_{g}(t)$ to be the residual excess at the sink.

Let $\operatorname{OPT}(G)$ denote the maximum possible value of any flow in network $G$. A flow $g$ in network $G$ is optimal if $|g|=\operatorname{OPT}(G)$ and $\xi$-optimal if $|g| \geq(1-\xi) \mathrm{OPT}(G)$. The generalized maximum flow problem is to find an optimal flow. The approximate generalized maximum flow problem is to find a $\xi$-optimal flow.

Size of numbers. We assume the capacities and initial excesses are given as integers between 1 and $B$, and the gains are given as ratios of integers which are between 1 and $B$. We assume $B \geq 2$, since otherwise the problem reduces to a traditional minimum cost flow problem. To simplify the running times we use $\tilde{\mathcal{O}}(f)$ to denote $f \log ^{\mathcal{O}(1)} m$.

\subsubsection{Generalized Residual Network}

We extend the definition of a residual network to generalized flows by appropriately accounting for the gain factors. Let $g$ be a generalized flow in network $G=$ 
$(V, E, s, u, \gamma, e)$. With respect to the flow $g$, the residual capacity function is defined by $u_{g}(v, w)=u(v, w)-g(v, w)$. The residual network is $G_{g}=\left(V, E, s, u_{g}, \gamma, e_{g}\right)$.

To gain intuition, we consider an example from the currency conversion problem that was described in Section 1.3. Let node $v$ represent U.S. dollars and let node $w$ represent French Francs. Suppose that we can convert up to 30 dollars into Francs at the exchange rate of 5 Francs per dollar, i.e., $u(v, w)=30, u(w, v)=0$, and $\gamma(v, w)=5$. If we convert $g(v, w)=20$ dollars, then we obtain 100 Francs. We can still convert up to $u_{g}(v, w)=10$ dollars into Francs at the same exchange rate. We can also unconvert any or all of the $u_{g}(w, v)=0-g(w, v)=g(v, w) / \gamma(v, w)=100$ Francs back into dollars at the symmetric exchange rate of $\gamma(w, v)=0.2$ dollars per Franc. Note that in general, the exchange rates are not symmetric, but here we are undoing a previous transaction, not creating a new one.

The following lemma is straightforward. It says that solving the problem in the residual network is equivalent to solving it in the original network.

Lemma 2.3.1. Let $g$ be a generalized flow in network $G$ and let $g^{\prime}$ be a generalized flow in the residual network $G_{g}$. Then $\operatorname{OPT}(G)=|g|+\operatorname{OPT}\left(G_{g}\right)$. Generalized flow $g^{\prime}$ is a generalized maximum flow in $G_{g}$ if and only if $g+g^{\prime}$ is a generalized maximum flow in $G$.

As before, we define $E_{g}=\left\{(v, w) \in E: u_{g}(v, w)>0\right\}$ to be the set of residual $\operatorname{arcs}$ in $G_{g}$. We also define residual arcs, paths, and cycles as before. A lossy network is a network in which each residual arc is lossy, i.e., no residual arc has gain factor exceeding one. 


\subsubsection{Relabeled Network}

With respect to generalized network $G=(V, E, t, u, \gamma, e)$, a labeling function is a function $\mu: V \rightarrow \Re_{>0} \cup\{\infty\}$ such that $\mu(t)=1$. We note that the node labels are the inverses of the linear programming dual variables, corresponding to the primal problem with decision variables $\{g(v, w):(v, w) \in E\}$. This idea of relabeling was originally introduced by Glover and Klingman [19]. Intuitively, node label $\mu(v)$ changes the local units in which flow is measured at node $v$; it is the number of old units per new unit. For example, in the currency conversion problem, if we change the basic unit of currency at node $v$ from U.S. dollars to pennies, then $\mu(v)=1 / 100$. To create an equivalent problem using the new units, we must appropriately normalize the capacity limits, gain factors, and initial excesses.

Continuing with the currency conversion example, suppose that we start with 1,700 dollars at node $v$, then in the new problem we start with 1,700,000 pennies. Similarly, if we could convert up to 800 dollars into Francs at the exchange rate of 5 Francs per dollar, then now we can convert up to 80,000 pennies into Francs at the exchange rate of 5/100 Francs per penny.

Thus, it is natural to define for each $(v, w) \in E$, the relabeled capacities, relabeled gains, and relabeled initial excesses by:

$$
\begin{array}{rlrl}
u_{\mu}(v, w) & =u(v, w) / \mu(v) & & \text { (relabeled capacity) } \\
\gamma_{\mu}(v, w) & =\gamma(v, w) \mu(v) / \mu(w) & & \text { (relabeled gain) } \\
e_{\mu}(v) & =e(v) / \mu(v) . & \text { (relabeled initial excess) }
\end{array}
$$


The relabeled network is denoted by $G_{\mu}=\left(V, E, t, u_{\mu}, \gamma_{\mu}, e_{\mu}\right)$. The following lemma is straightforward. It says that the relabeled network is an equivalent instance of the generalized maximum flow problem.

Lemma 2.3.2. For any labeling function $\mu, g$ is a generalized flow in network $G$ if and only if $g_{\mu}(v, w)=g(v, w) / \mu(v)$ is a generalized flow of the same value $|g|$ in network $G_{\mu}$.

By relabeling the residual network, we can create new equivalent instances of the generalized maximum flow problem. With respect to a flow $g$ and labels $\mu$, we define the relabeled residual capacities and relabeled residual excesses by:

$$
\begin{aligned}
u_{g, \mu}(v, w) & =u_{g}(v) / \mu(v) & \text { (relabeled residual capacity) } \\
e_{g, \mu}(v) & =e_{g}(v) / \mu(v) . & \text { (relabeled residual excess) }
\end{aligned}
$$

The relabeled residual network is denoted by $G_{g, \mu}=\left(V, E, t, u_{g, \mu}, \gamma_{\mu}, e_{g, \mu}\right)$.

Canonical Labels. We define the canonical label of a node $v$ in network $G$ to be the inverse of the highest gain residual path from $v$ to the sink. If no such path exists, its label is $\infty$. If $G$ has no residual flow-generating cycles, then the canonical labels can be determined using a single Bellman-Ford shortest path computation with lengths $l=-\log \gamma$. If $G$ is a lossy network, then a Dijkstra shortest path computation suffices. 


\subsubsection{Flow Decomposition}

A traditional pseudoflow can be decomposed into a collection of at most $m$ paths and cycles. In this section, we show how a generalized pseudoflow $g$ can be decomposed into a small collection of "elementary" generalized pseudoflows that conform to $g$. By conform, we mean that the elementary pseudoflows can only be positive on arcs on which $g$ is positive. In addition, the elementary pseudoflow can only generate excess (deficit) at a node for which $g$ generates excess (deficit).

This decomposition is useful to characterize the optimality conditions for the generalized maximum flow problem. It can also simplify a generalized flow by eliminating flow which does not reach the sink; this enables us to convert a flow into a proper flow of the same value. Also, it leads to an alternate "path-based formulation" of the problem.

We denote the gain of a cycle (path) $\Gamma$ by $\gamma(\Gamma)=\prod_{e \in \Gamma} \gamma(e)$. A unit-gain cycle has gain equal to one. A flow-generating (flow-absorbing) cycle is a cycle with gain more (less) than one. We remark if we use the logarithmic cost function $c=-\log \gamma$, then flow-generating cycles are in one-to-one correspondence with negative cost cycles.

There are five types of elementary generalized pseudoflows:

- Type I (path): It is positive only on the arcs of a path. It only creates deficit at the first node of the path and excess at the last node.

- Type II (unit-gain cycle): It is positive only on the arcs of a unit gain cycle. It does not create excess or deficit. 
- Type III (cycle-path): It is positive only on the arcs of a flow-generating cycle and a (possibly trivial) path connecting the cycle to a node. It only creates excess at the endpoint of the path.

- Type IV (path-cycle): It is positive only on the arcs of a flow-absorbing cycle and a (possibly trivial) path from a node to the cycle. It only creates deficit at the endpoint of the path.

- Type $V$ (bicycle): It is positive only on the arcs of a flow-generating cycle, a flow-absorbing cycle and a (possibly trivial) path connecting the two cycles. It does not create excess or deficit.

The following theorem is due to Gondran and Minoux [29]. We repeat the proof from $[20]$.

Theorem 2.3.3. For every pseudoflow $g$, there exists a collection of $k \leq m$ elementary pseudoflows $g_{1}, \ldots, g_{k}$ that conform to $g$ such that $g(v, w)=\sum_{i} g_{i}(v, w)$. Such a decomposition can be found in $\mathcal{O}(m n)$ time.

Proof. We prove by induction on the number of arcs with positive flow. Let $G^{\prime}$ be the subgraph of $G$ consisting of arcs with positive flow.

If $G^{\prime}$ is acyclic then we can trace the flow from any deficit node to some excess node along a path, and subtract flow on the path until one arc on the path has zero flow. The subtracted flow is of Type I, and the theorem follows by induction.

Otherwise, let $\Gamma$ be a cycle in $G^{\prime}$. If $\gamma(\Gamma)=1$, then we can subtract flow around the cycle until one arc on the cycle has zero flow. The subtracted flow is of Type II, and the theorem follows by induction. 
Otherwise, suppose $\Gamma$ is a flow-generating cycle (the case when $\Gamma$ is a flowabsorbing cycle is similar). We subtract flow around the cycle until one arc on the cycle has zero flow, and let $h$ denote the flow removed. This reduces the excess at one of the nodes, say $v$, possibly to a negative value. If node $v$ no deficit after reducing flow around the cycle, then the subtracted flow is of Type III (with a trivial path), and the theorem follows by induction. Otherwise we decompose $g-h$ inductively. Now, since $v$ has deficit, the decomposition of $g-h$ includes components of Type I or IV that are responsible for creating all of the deficit at node $v$. Each of these components, together with an appropriate fraction of $h$, corresponds to a component of Type III or $\mathrm{V}$ in the decomposition of $g$. If node $v$ originally had positive excess in $G$, then there will be some fraction of $h$ left over; this is a Type III pseudoflow (with a trivial path).

The above procedure strictly decreases the number of arcs with positive flow in amortized $\mathcal{O}(n)$ time.

An augmenting path is a residual path from a node with excess to the sink. It corresponds to a Type I pseudoflow whose first node has excess in the original network, and whose last node is the sink.

Corollary 2.3.4. Let $G$ be a lossy network. Then, there exists a generalized maximum flow that is positive on at most $m$ augmenting paths.

Proof. Let $g^{*}$ be a generalized maximum flow. We decompose it according to Theorem 2.3.3. Note that there are no Type III or V components in the decomposition, since $G$ is a lossy network. We remove all components that do not generate excess 
at the sink, and denote the resulting flow by $g$. This removes all Type II and IV components, and also some Type I components. The only components that remain are Type I components that generate excess at the sink. These correspond to augmenting paths. It follows that $g$ is also optimal and can be decomposed into at most $m$ augmenting paths.

A generalized augmenting path (GAP) is a residual flow-generating cycle, together with a (possibly trivial) residual path from a node on this cycle to the sink. It corresponds to a Type III pseudoflow whose path ends at the sink.

Corollary 2.3.5. Let $G$ be a generalized network. Then, there exists a generalized maximum flow that is positive on at most $m$ augmenting paths or GAPs.

Proof. Let $g^{*}$ be a generalized maximum flow. We decompose it according to Theorem 2.3.3. We remove all components that do not generate excess at the sink, and denote the resulting flow by $g$. As above, the only Type I components that can generate excess at the sink correspond to augmenting paths. The only Type III components that generate excess at the sink correspond to GAPs. The corollary immediately follows.

Recall, a generalized flow is allowed to generate excesses, but no deficits. A proper flow does not generate any additional excesses, except possibly at the sink.

Corollary 2.3.6. Let $g$ be a flow in network $G$. Then in $\mathcal{O}(m n)$ time we can find a proper flow $g^{\prime}$ in $G$ such that $\left|g^{\prime}\right| \geq|g|$. 
Proof. Let $g$ be a flow. We decompose it according to Theorem 2.3.3. As above, the only components that generate excess at the sink correspond to augmenting paths and GAPs. Moreover, these components do not generate excess at any other node. Let $g^{\prime}$ be the flow induced by only these useful components. Now $g^{\prime}$ is a proper flow and $\left|g^{\prime}\right| \geq|g|$.

Path-Based Formulation. Now, we consider an alternate path-based formulation for the generalized maximum flow problem in lossy networks. In this formulation, we have a nonnegative variable $x(P)$ for each augmenting path $P$, representing the amount of flow sent along $P$. We also include a capacity constraint for each arc. The objective is to maximize the net flow into the sink: $\sum_{P \text { augmenting path }} \gamma(P) x(P)$. Corollary 2.3.4 implies that the path formulation is equivalent to the "arc-based" formulation considered in Section 2.3. We note that the path-based formulation may have exponentially many variables.

\subsubsection{Optimality Conditions}

Let $g$ be a generalized flow in network $G$. If $G_{g}$ has an augmenting path, then we can improve $g$ by augmenting flow along such a path. By augmenting flow, we mean increasing the flow of forward arcs along the residual path (and decreasing flow on the reverse arcs to maintain generalized antisymmetry), while conserving flow at intermediate nodes of the path. If one unit of flow is sent from node $v$ to the sink along augmenting path $P$, then $\gamma(P)$ units arrive at $t$. 
If $G_{g}$ has a GAP, then we can improve $g$ by augmenting flow along such a GAP. If one unit of flow is sent from a node $v$ around a residual flow-generating cycle, then more than one unit arrives back at $v$. By sending flow around such a cycle, we can increase the residual excess at any node of the cycle, while conserving flow at all other nodes. This excess can subsequently be sent along a residual path to the sink, which increases the excess at $t$.

The following theorem of Onaga [44] says that these are the only two ways to improve the current flow $g$. It generalizes Theorem 2.2.1.

Theorem 2.3.7. A generalized flow $g$ is a generalized maximum flow if and only if $G_{g}$ has no augmenting paths or GAPs.

Proof. Clearly if a generalized flow $g$ has an augmenting path or GAP in $G_{g}$ then it is not optimal.

Now, suppose $g$ has no augmenting paths or GAPs in $G_{g}$. Let $g^{*}$ be a generalized maximum flow. Decompose $g^{*}-g$ according to Theorem 2.3.3. Since $g$ has no augmenting paths or GAPs, there are none in the decomposition either. These are the only two elementary pseudoflows that can generate excess at the sink. Hence $g$ is optimal.

The following theorem expresses the optimality conditions in terms of node labels.

Theorem 2.3.8. A generalized flow $g$ is a generalized maximum flow if and only if there exists labels $\mu$ such that:

$$
\forall(v, w) \in G_{g}: \quad \gamma_{\mu}(v, w) \leq 1
$$


$\forall v$ that cannot reach $t$ in $G_{g}: \quad \mu(v)=\infty$.

Proof. The proof is immediate from linear programming duality; the node labels are the inverses of the dual variables. We now give a direct combinatorial proof.

Suppose $g$ is a generalized maximum flow. Let $\mu$ be the canonical labels in $G_{g}$. Let $T$ be the set of nodes that can reach the sink using residual arcs in $G_{g}$. Since $G_{g}$ has no GAPs, $T$ has no flow-generating cycles. Consequently, the labels are well-defined and can be computed efficiently using a Bellman Ford shortest path computation with lengths $l=-\log \gamma$. As a result, the labels satisfy $\mu(w) \geq$ $\gamma(v, w) \mu(v)$ for residual arcs in the subgraph of $G_{g}$ induced by nodeset $T$.

By definition of the canonical labels, all labels are positive, $\mu(t)=1$, and $\mu(v)=$ $\infty$ for all nodes $v \in V \backslash T$. Thus $\mu$ satisfies (2.4) and (2.5).

Now, suppose generalized flow $g$ and labels $\mu$ satisfy (2.4) and (2.5). Then, there can be no residual paths from excess nodes to the sink. Also the gain of a cycle is equal to the relabeled gain of a cycle; thus, there are no residual flowgenerating cycles involving nodes that can reach the sink. Thus, by Theorem 2.3.7, $g$ is optimal.

\subsection{Canceling All Flow-Generating Cycles}

In this section we briefly review a procedure for "canceling all flow-generating cycles." It is described in detail in Chapter 8. Many generalized flow computations can be performed much more efficiently in networks without residual flow-generating cycles. To overcome this obstacle, we send flow around a residual flow-generating 
cycle until one (or more) arcs become saturated. In the process additional excesses, but no deficits, may be created. This operation is called canceling a flow-generating cycle. The goal is to repeatedly cancel residual flow-generating cycles, until no such cycles remain. Goldberg, Plotkin, and Tardos [20] proposed an efficient method that is based on the CANCEL-AND-TIGHTEN algorithm of Goldberg and Tarjan [23]. Their algorithm requires $\tilde{\mathcal{O}}(m n \min \{m, n \log B\})$ time.

\subsection{Nearly-Optimal Flows}

The optimality conditions for the generalized maximum flow problem are characterized by Theorem 2.3.7 and Theorem 2.3.8. We now give conditions under which a flow is essentially optimal. Given a generalized flow $g$ in network $G$, the excess discrepancy is the difference between the value of the optimal flow and the current flow, i.e., $\operatorname{OPT}(G)-|g|$. The following lemma from [20] says that if the excess discrepancy is very small and there are no residual flow-generating cycles, then the flow can be "rounded" to an optimal solution. We give a modification of their proof.

Lemma 2.5.1. Let $g$ be a flow in network $G$ such that $\mathrm{OPT}(G)-|g|<B^{-m}$ and $G_{g}$ has no residual flow-generating cycles. Then, we can find an optimal flow in $\mathcal{O}(\mathrm{MF}(m, n))$ time.

Proof. Without loss of generality, we assume that there is a residual path in $G_{g}$ from every node to the sink; otherwise we could delete such useless nodes. Let $\mu$ be the canonical labels in $G_{g}$. Since $G_{g}$ has no residual flow-generating cycles, the labels are well defined. We find a flow $g^{*}$ that satisfies the complementary slackness 
conditions (2.4) with $\mu$, and, among all such flows, maximizes the excess at the sink. We will argue that $g^{*}$ is optimal (and hence $\mu$ is dual optimal). The procedure for determining $g^{*}$ is given in Figure 2.1 and is described below.

Input: network $G$, flow $g$ such that $G_{g}$ has no residual flow-generating cycles and $\operatorname{OPT}(G)-|g|<B^{-m}$

Output: maximum flow $g^{*}$ $\mu \leftarrow$ canonical labels in $G_{g}$ $h \leftarrow$ flow that saturates all arcs with relabeled gain above one, and sends zero flow on all arcs with relabeled gain equal to one $G_{g, \mu}^{\prime} \leftarrow$ subgraph of $G_{g, \mu}$ induced by gain one arcs Consider excess nodes as sources with capacity $e_{g, \mu}(v)$ and deficit nodes as sinks with demand $\left|e_{g, \mu}(v)\right|$. Compute flow $f^{\prime}$ in $G_{g, \mu}^{\prime}$ that satisfies all demand and maximizes net flow into $t$

$g^{*}(v, w) \leftarrow h(v, w)+\mu(v) f^{\prime}(v, w)$

Figure 2.1: Rounding to an Optimal Flow

Let $h$ denote the pseudoflow that satisfies (2.4) and sends zero flow on every arc with unit relabeled gain. The relabeled node excess of pseudoflow $h$ at node $v$ is:

$$
e_{h, \mu}(v)=e_{\mu}(v)-\sum_{w:(v, w) \in E} h_{\mu}(v, w)=e_{\mu}(v)-\sum_{w:(v, w) \in E, \gamma_{\mu}(v, w)>1} u_{\mu}(v, w) .
$$

The original capacities and excesses are integral. The canonical labels are integral multiples of $L^{-1}$, where $L$ is the least common denominator of the gains of paths in $G$. Thus, the relabeled excesses and capacities are also integral multiples of $L^{-1}$. In particular, $\left|h_{\mu}\right|=e_{h, \mu}(t)$ is an integral multiple of $L^{-1}$. 
The procedure finds a generalized flow $g^{*}$ that maximizes the excess at the sink among all flows that satisfy (2.4). To find such a flow, let $G_{g, \mu}^{\prime}$ denote the subgraph of $G_{g, \mu}$ induced by gain one arcs. We view excess nodes in $G_{g, \mu}$ as sources with capacity $e_{g, \mu}(v)$ and deficit nodes as sink nodes with demand $\left|e_{g, \mu}(v)\right|$. Let $f_{\mu}=f^{\prime}$ be a traditional flow in $G_{g, \mu}^{\prime}$ that satisfies all of the demand, and maximizes the net flow into the sink. Note that such a flow is guaranteed to exists, since the restriction of $g_{\mu}$ to gain one arcs in $G_{g, \mu}$ is such a flow. We can compute $f_{\mu}$ using a traditional maximum flow computation. By the integrality theorem for the maximum flow problem, since all of the demands and capacities are integral multiples of $L^{-1}$, then so is the value of the maximum flow $\left|f_{\mu}\right|$. Let $g^{*}=h+f$, where $f$ is the "unrelabeled" version of $f_{\mu}$. Now $\left|g^{*}\right| \geq|g|$ Thus OPT $(G)-\left|g^{*}\right|<B^{-m}$. Since $\left|g^{*}\right|=|h|+|f|=\left|h_{\mu}\right|+\left|f_{\mu}\right|$ and $\operatorname{OPT}(G)$ are both integral multiples of $L^{-1}$, and $L \leq B^{m}$, it follows that $g^{*}$ is optimal.

The next lemma indicates that if a generalized flow is $\xi$-optimal for sufficiently small $\xi$, then it is essentially optimal. It is used to provide termination of our exact algorithms.

Lemma 2.5.2. Let $g$ be a $B^{-3 m}$-optimal flow in network $G$. Then we can compute an optimal flow in $G$ in $\tilde{\mathcal{O}}(m n \min \{m, n \log B\})$ time.

Proof. Let $g$ be a $B^{-3 m}$-optimal flow. Then the excess discrepancy of $g$ is

$$
\operatorname{OPT}(G)-|g| \leq B^{-3 m} \operatorname{OPT}(G) \leq B^{-m}
$$


The last inequality follows since each arc entering the sink has capacity and gain each at most $B$; therefore $\mathrm{OPT}(G) \leq m B^{2} \leq B^{m+1}$. The lemma then follows from Lemma 2.5.1 provided $G_{g}$ has no residual flow-generating cycles.

If there are residual flow-generating cycles in $G_{g}$, then we can cancel them in the stated time bound, as described in Section 2.4. Canceling flow-generating cycles can only create additional excesses. Thus, the resulting flow is also $B^{-3 m}$-optimal, and the previous argument applies. 


\section{Chapter 3}

\section{Generalized Maximum Flow}

\section{Literature}

In this chapter we review the generalized maximum flow literature. Since the generalized flow problem is a special case of linear programming, it can be solved by general purpose linear programming techniques. The problem can also be solved by combinatorial methods, which have led to superior algorithm for many traditional network flow problems. In the 1960's Jewell [33] and Onaga [44] proposed exponential-time augmenting path algorithm for the generalized maximum flow problem. It wasn't until the late 1980's that Goldberg, Plotkin and Tardos [20] designed the first polynomial-time combinatorial algorithms for the generalized maximum flow problem. 


\subsection{Combinatorial Methods}

Exact algorithms. The first combinatorial algorithms for the generalized maximum flow problem were exponential-time augmenting path algorithms proposed by Jewell [33] and Onaga [44]. The generalized maximum flow problem is closely related to the traditional minimum cost flow problem. Truemper [55] observed that by using the cost function $c=-\log \gamma$, many of the early generalized maximum flow algorithms were, in fact, analogs of pseudo-polynomial minimum cost flow algorithms. Jewell's [33] generalized maximum flow algorithm is an analog of Klein's [40] cyclecanceling algorithm for the minimum cost flow problem. Similarly, Onaga's [44] algorithm is analogous to the successive shortest path algorithm developed independently by Busacker and Gowen [7], Iri [30], and Jewell [32]. Truemper's [54] algorithm is an analog of Ford and Fulkerson's [16] primal-dual algorithm. Jarvis and Jezior's [31] algorithm is an analog of Fulkerson's [18] out-of-kilter algorithm.

Goldberg, Plotkin, and Tardos [20] designed the first two polynomial-time combinatorial algorithms for the generalized maximum flow problem: Fat-Path and MCF. They also developed much of the machinery used in subsequent generalized flow algorithms. The Fat-Path algorithm maintains a generalized flow and uses capacity-scaling. The main idea is to repeatedly send along "fat-paths", i.e., paths that have enough capacity to generate large amounts of excess at the sink. Periodically, flow is rerouted to more efficient paths by "cancelling all flow-generating cycles." The algorithm requires $\tilde{\mathcal{O}}\left(m^{2} n^{2} \log ^{2} B\right)$ time. It is described in detail in Chapter 7 . 
Algorithm MCF maintains a pseudoflow with no excess nodes, except the source. It repeatedly performs a traditional minimum cost flow computation with cost function $c=-\log \gamma$. It interprets the result as an augmentation from the source to deficit nodes in the generalized network. It requires $\tilde{\mathcal{O}}\left(m^{2} n^{2} \log B\right)$ time.

Radzik [49] modified the Fat-Path algorithm and improved the complexity to $\tilde{\mathcal{O}}\left(m^{3} \log B+m^{2} n \log B \log \log B\right)$. The bottleneck computation in the original FatPath algorithm is cancelling flow-generating cycles. Radzik reduces this bottleneck by only canceling flow-generating cycles that have sufficiently large gain. Since there are still residual flow-generating cycles, finding fat-paths becomes much more complicated.

Goldfarb and Jin [26] designed an algorithm based on the MCF algorithm. It matches the complexity of the original MCF algorithm, without using the dynamic tree data structure. Instead of using a traditional minimum cost flow computation at each iteration, they augment flow along highest-gain paths. They introduced the concept of "arc deficits" to ensure that most of the augmentations deliver a sufficiently large amount of flow to deficit nodes. Goldfarb, Jin, and Orlin [28] proposed an $\tilde{\mathcal{O}}\left(m^{3} \log B\right)$ algorithm motivated by the Fat-Path algorithm. Their algorithm uses "arc excesses", much in the same way that the MCF variant uses arc deficits.

Subsequent to my thesis, Wayne [58] developed the first efficient primal algorithm for the problem; it repeatedly sends flow along "minimum ratio" augmenting paths or GAPs. His algorithm also extends to solve the generalized minimum cost 
flow problem; it is the first polynomial-time algorithm for the problem that is not based on general linear programming techniques.

Approximation algorithms. Researchers have also developed algorithms for the approximate generalized maximum flow problem. Cohen and Megiddo [10] designed the first strongly polynomial-time approximation algorithm for the generalized maximum flow problem. There method is based on solving the linear programming dual. If there are no capacity constraints, then the dual has two variables per inequality (TVPI). Given an arbitrary cost function, a minimum cost generalized flow in an uncapacitated network with only sink nodes can be determined using a subroutine that tests the feasibility of a TVPI system. To solve the generalized maximum flow problem, Cohen and Megiddo iteratively relax the capacity constraints and introduce a cost function chosen to respect the capacities. The minimum cost generalized flow in the uncapacitated network is scaled to a feasible one, and the process is repeated in the residual network.

Subsequently, Radzik [48] observed that the Fat-Path algorithm computes a $\xi$-optimal flow in $\tilde{\mathcal{O}}\left(m n^{2} \log B\right) \log (1 / \xi)$ time. He also gave a strongly polynomialtime algorithm for canceling all flow-generating cycles; this implies that the FatPath algorithms finds an approximate flow in $\tilde{\mathcal{O}}\left(m^{2} n\right) \log (1 / \xi)$ time. Radzik [49] Fat-Path variant, that cancels only sufficiently high gain flow-generating cycles, runs in $\tilde{\mathcal{O}}\left(m^{2}+m n \log \log B\right) \log (1 / \xi)$ time.

Subsequent to my thesis, Oldham [43] and Wayne and Fleischer [59] designed approximation algorithms for generalized flow problems using an exponential length 
function in a packing framework. The algorithm of [43] requires $\tilde{\mathcal{O}}\left(m^{2} n^{2} \epsilon^{-2}\right)$ time, and the algorithm of [59] requires $\tilde{\mathcal{O}}\left(m^{2} \epsilon^{-2}\right)$. Wayne and Fleischer also obtain a $\tilde{\mathcal{O}}\left(m^{2}+m n \log \log B\right) \log (1 / \epsilon)$ time complexity bound using the gain-scaling methodology presented in this thesis. It is interesting to note that the packing techniques also extend to approximately solve generalized versions of the minimum cost flow and multicommodity flow problems.

\subsection{Linear Programming Methods}

The generalized maximum flow problem can be solved by general purpose linear programming methods including simplex, ellipsoid and interior point. Researchers have tailored some of these methods specifically for the generalized flow problem.

Simplex. Dantzig [12] proposed the generalized network simplex method; it is a specialization of the simplex method which exploits the topological structure of the basis, much like the network simplex method does for the minimum cost flow problem, e.g. see [1]. For traditional networks, each linear programming basis corresponds to a spanning tree. For generalized networks, each basis corresponds to a node-disjoint collection of good 1-trees that span all nodes. A 1-tree is a tree plus one additional arc creating a unique cycle. If the cycle does not have unit-gain, it is called good.

Finite termination can be guaranteed by using a general pivot rule like Bland's rule [6, 9]. Elam, Glover, and Klingman [14] gave a combinatorial primal simplex pivot rule that guarantees finiteness. Goldfarb and Jin [25] designed the first 
polynomial-time (dual) simplex algorithm for the problem; it is based upon their polynomial-time combinatorial algorithm in [26]. Very recently, Goldfarb, Jin, and Lin [27] developed a faster dual simplex algorithm for the problem; it is based on the combinatorial algorithm in [28].

Interior point. Karmarkar [37] and Renegar [50] discovered polynomial-time interior point methods for linear programming. Kapoor and Vaidya [36] showed how to speed up these interior-point methods on network flow problems, by exploiting the structured sparsity in the underlying constraint matrix. For the generalized maximum flow problem, these algorithms run in $\mathcal{O}\left(m^{1.5} n^{2.5} \log B\right)$ time. Using fast matrix multiplication, Vaidya [57] improved the worst-case complexity to $\mathcal{O}\left(m^{1.5} n^{2} \log B\right)$. Murray [42] designed a different interior-point algorithm of the same complexity without using theoretically fast matrix multiplication. Kamath and Palmon [35] matched the complexity of the above two algorithm by considering a closely related quadratic programming problem. We note that these algorithms can also solve the generalized minimum cost flow problem in the same time bound and they can be extended to solve multicommodity flow versions. It is not known how to improve the worst-case complexity of these exact interior point algorithms to find approximate flows.

\subsection{Best Complexity Bounds}

Currently, the best worst-case complexity bounds for the generalized maximum flow problems are $\mathcal{O}\left(m^{1.5} n^{2} \log B\right)$ due to Vaidya [57] and $\tilde{\mathcal{O}}\left(m^{3} \log B\right)$ due to Goldfarb, 
Jin, and Orlin [28]. For the generalized maximum flow problem, the best known complexity bounds for computing $\xi$-optimal flows are $\tilde{\mathcal{O}}\left(m^{2}+m n \log \log B\right) \log (1 / \xi)$ of Radzik [49], $\tilde{\mathcal{O}}\left(m^{2} n\right) \log (1 / \xi)$ of Radzik [48], and, $\tilde{\mathcal{O}}\left(m^{2} \xi^{-2}\right)$ of Wayne and Fleischer [59]. The existence of a strongly-polynomial algorithm for the generalized maximum flow problem remains a challenging open question. 


\section{Chapter 4}

\section{Gain-Scaling}

Scaling is a powerful technique for deriving polynomial-time algorithms for a wide variety of combinatorial optimization problems. It was first introduced by Edmonds

and Karp [13] for the maximum flow problem. Almost all of the best traditional network flow algorithms use some form of capacity or cost scaling. In this chapter we introduce a gain-scaling method. This new technique can be used to design several new polynomial-time generalized flow algorithms. Our method rounds down the gain factors and solves the problem in the rounded network. Then if necessary, it repeatedly refines the flow, until it obtains a solution of the desired level of precision. The benefit is that generalized flow problems are often much easier to solve in the resulting rounded networks. 


\subsection{Rounding Down the Gains}

In our algorithms we round down the gains so that they are all integer powers of a base $b=(1+\xi)^{1 / n}$. Our rounding scheme applies in lossy networks. We round the gain of each residual arc down to $\bar{\gamma}(v, w)=b^{c(v, w)}$ where $c(v, w)=\left\lfloor\log _{b} \gamma(v, w)\right\rfloor$. To maintain antisymmetry we set $\bar{\gamma}(w, v)=1 / \bar{\gamma}(v, w)$. Note that if both $(v, w)$ and $(w, v)$ are residual arcs then each arc has unit gain, and the definition is consistent. Let $H$ denote the resulting $\xi$-rounded network. Note that $H$ is a lossy network and shares the same set of residual arcs as $G$. Let $C=\max _{e \in E} c(e)$ and note that

$$
C \leq 1+\log _{b} B=1+\frac{\log B}{\log (1+\xi)^{1 / n}} \leq 1+\frac{n \log B}{\xi}
$$

Let $h$ be a flow in network $H$. To convert to a flow in network $G$, we define the interpretation of a flow $h$ in network $H$ into a flow $g$ in network $G$ by:

$$
g(v, w)=\left\{\begin{array}{ll}
h(v, w) & \text { if } g(v, w) \geq 0 \\
-\gamma(w, v) h(w, v) & \text { if } g(v, w)<0 .
\end{array} \quad\right. \text { (flow interpretation) }
$$

That is, flow $g$ agrees with flow $h$ on arcs with positive flow, but, to maintain antisymmetry, flow $g$ may differ from $h$ on the reversals of these arcs. Note that flow interpretation may create additional excesses, but no deficits. We give an illustrative example of flow interpretation in Figure 4.1. Suppose that in the rounded network we send 100 units of flow through arc $(v, w)$, and we route 50 units on the top path and 30 units on the bottom path. Then, in the original network, we do the same. This creates residual excess at node $w$, since the original gain factor was rounded down. 
Flow in rounded network:
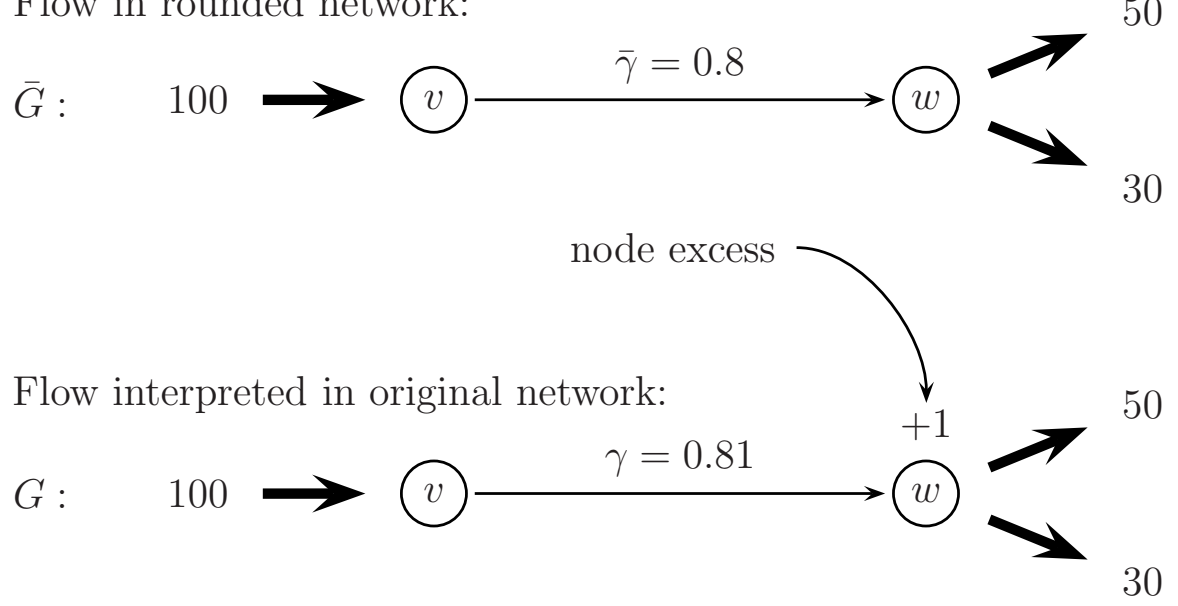

Figure 4.1: Flow Interpretation

At times, we want to apply the rounding scheme to networks with residual flowgenerating cycles. To do this, we first cancel all residual flow-generating cycles, as described in Section 8.1 and canonically relabel the network. After the relabeling, the relabeled gain numerators and denominators might be as big as $B^{n}$. In this case

$$
C \leq 1+\log _{b} B^{2 n}=1+\frac{2 n \log B}{\log (1+\xi)^{1 / n}} \leq 1+\frac{2 n^{2} \log B}{\xi} .
$$

We show that approximate flows in the rounded network induce approximate flows in the original network. The next theorem says that the rounded network is close to the original network.

Theorem 4.1.1. Let $G$ be a lossy network and let $H$ be the rounded network constructed as above. If $0<\xi<1$ then $(1-\xi) \operatorname{OPT}(G) \leq \mathrm{OPT}(H) \leq \mathrm{OPT}(G)$.

Proof. Clearly $\mathrm{OPT}(H) \leq \mathrm{OPT}(G)$ since we only decrease the gain factors of residual arcs. We consider the path formulation of the generalized maximum flow problem in lossy networks, as described in Section 2.3.3. Recall, in this formulation, 
there is a variable $x_{P}$ for each augmenting path $P$, representing the amount of flow sent along this path. Let $x^{*}$ be an optimal path flow in $G$. Then $x^{*}$ is also a feasible path flow in $H$. From augmenting path $P, \gamma(P) x_{P}^{*}$ units of flow arrive at the sink in network $G$, while only $\bar{\gamma}(P) x_{P}^{*}$ arrive in network $H$. The theorem the follows, since for each augmenting path $P$,

$$
\bar{\gamma}(P) \geq \frac{\gamma(P)}{b^{|P|}} \geq \frac{\gamma(P)}{b^{n}}=\frac{\gamma(P)}{1+\xi} \geq \gamma(P)(1-\xi)
$$

Corollary 4.1.2. Let $G$ be a lossy network and let $H$ be the $\xi$-rounded network. If $0<\xi<1$, then the interpretation of a $\xi^{\prime}$-optimal flow in $H$ is a $\xi+\xi^{\prime}$-optimal flow in $G$.

Proof. Let $h$ be a $\xi^{\prime}$-optimal flow in $H$. Let $g$ be the interpretation of flow $h$ in $G$. Then

$$
|g| \geq|h| \geq\left(1-\xi^{\prime}\right) \operatorname{OPT}(H) \geq(1-\xi)\left(1-\xi^{\prime}\right) \operatorname{OPT}(G) \geq\left(1-\xi-\xi^{\prime}\right) \operatorname{OPT}(G) .
$$

The third inequality follows from Theorem 4.1.1.

\section{$4.2 \quad$ Error-Scaling}

In this section we show how error-scaling can be used to speed up computations for generalized flow problems. We use the idea to convert constant-factor approximation algorithms in fully polynomial-time approximation schemes. We also use the technique to improve the complexity of our Fat-Path variant, when finding nearly 
optimal and optimal flows; Radzik [49] used the error-scaling in a similar manner for his Fat-Path variant.

Suppose we have a subroutine which finds a 1/2-optimal flow in network $G$. Error-scaling enables us to determine a $\xi$-optimal flow in network $G$ by calling this subroutine $\log (1 / \xi)$ times. To accomplish this we first find a $1 / 2$-optimal flow $g$ in network $G$. Then we find a $1 / 2$-optimal flow $h$ in the residual network $G_{g}$. Now $g+h$ is a $1 / 4$-optimal flow in network $G$, since each call to the subroutine captures at least half of the remaining flow. In general, we can find a $\xi$-optimal flow with $\log (1 / \xi)$ calls to the subroutine.

Now, we give a divide-and-conquer version of error-scaling. It allows us to recursively combine two $\sqrt{\xi}$-optimal into a $\xi$-optimal flow. In some applications, this scheme leads to faster algorithms.

Lemma 4.2.1. Let $g$ be a $\sqrt{\xi}$-optimal flow in network $G$. Let $h$ be a $\sqrt{\xi}$-optimal flow in $G_{g}$. Then the flow $g+h$ is $\xi$-optimal in $G$.

Proof.

$$
\begin{aligned}
\operatorname{OPT}(G)-|g+h| & =\operatorname{OPT}\left(G_{g}\right)-|h| \\
& \leq \sqrt{\xi} \operatorname{OPT}\left(G_{g}\right) \\
& =\sqrt{\xi}(\operatorname{OPT}(G)-|g|) \\
& \leq \xi \operatorname{OPT}(G) .
\end{aligned}
$$

The two inequalities follows from $\sqrt{\xi}$-optimality of $g$ and $h$, respectively. 


\section{Chapter 5}

\section{Augmenting Path Algorithms}

In this chapter, we first review the augmenting path algorithms of Onaga [44] and Truemper [55]. These are among the simplest algorithms known for the generalized maximum flow problem, but require exponential time.

Our variant runs Truemper's algorithm in an appropriately rounded network. It is the simplest and cleanest known polynomial-time approximation algorithm for the problem. By incorporating error-scaling and canceling flow-generating cycles, our variant can also be used to compute optimal flows.

\subsection{Augmenting Path Algorithm}

A natural and intuitive algorithm for the generalized maximum flow problem in lossy networks is to repeatedly send flow from excess nodes to the sink along highest-gain (most-efficient) augmenting paths. This is essentially Onaga's [44] algorithm. It

generalizes Ford and Fulkerson's [15] augmenting path algorithm for the traditional 
maximum flow problem. It is also the generalized flow analog of the successive shortest path algorithm for the traditional minimum cost flow problem, which was developed independently by Jewell [32], Iri [30], and Busacker and Gowen [7]. Onaga observed that if the input network has no residual flow-generating cycles, then the algorithm maintains this property. Thus, we can find a highest-gain augmenting path using a single shortest path computation with lengths $l=-\log \gamma$. By maintaining canonical labels, we can ensure that the relabeled residual network remains lossy. Thus, all arc lengths are nonnegative, and a Dijkstra shortest path computation suffices. Unit gain paths in the canonically relabeled network correspond to highest gain paths in the original network. Upon termination, the resulting flow is optimal by Theorem 2.3.7. However, Onaga's algorithm may require exponentialtime and may not terminate if the data are real-valued.

\subsection{Primal-Dual Algorithm}

In this section we review Truemper's [55] primal-dual algorithm for the generalized maximum flow problem. It is an implementation of Onaga's augmenting path algorithm that is guaranteed to terminate in finite time, even for real-valued data. It is also the primal-dual algorithm for linear programming applied to the generalized maximum flow problem: the algorithm maintains a primal feasible flow $g$ and dual (infeasible) canonical labels $\mu$ that satisfy the complementary slackness optimality conditions:

$$
\forall(v, w) \in G_{g}: \quad \gamma_{\mu}(v, w) \leq 1 .
$$


The dual solution is infeasible because some excess nodes have positive canonical labels. At each iteration, the algorithm decreases this dual infeasibility, while preserving complementary slackness. It can also be viewed as a generalized flow analog of Ford and Fulkerson's [16] primal-dual algorithm for the traditional minimum cost flow problem, which repeatedly sends flow along all cheapest paths, using a single maximum flow computation.

Onaga's algorithm repeatedly sends flow along a single highest-gain augmenting path at a time. In contrast, Truemper's primal-dual algorithm sends flow along all highest-gain augmenting paths simultaneously, using a single traditional maximum flow computation. The algorithm is given in Figure 5.1.

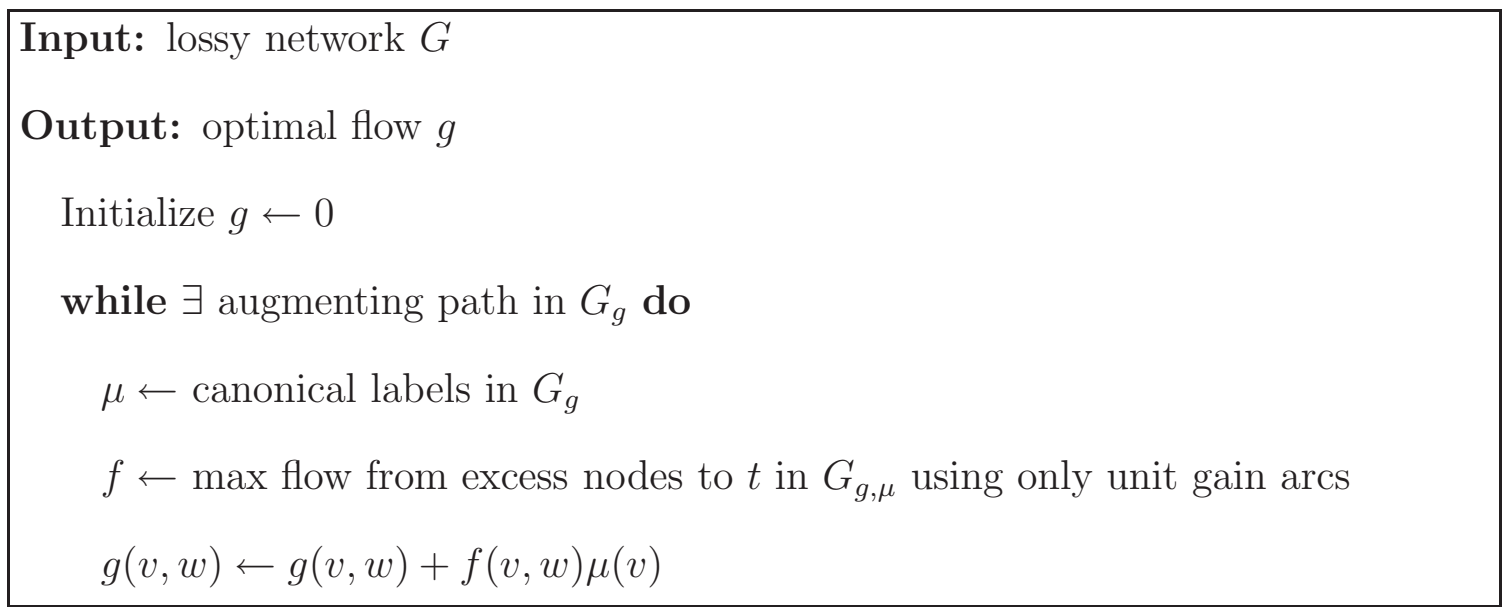

Figure 5.1: Algorithm Primal-Dual

The following invariant ensures that the residual network has no flow-generating cycles; this implies that the canonical labels can be recomputed efficiently.

Invariant 5.2.1. Throughout the primal-dual algorithm, the canonically relabeled network $G_{g, \mu}$ is a lossy network. 
Proof. We prove by induction on the number of iterations. The input to the primaldual algorithm is assumed to be a lossy network. At the beginning of an iteration, the network is canonically relabeled. Flow is sent only along arcs with relabeled gain factors equal to one. So, only the reversals of these arcs can be added to the residual network, and all of these reverse arcs have relabeled gain factors of one. Thus, the resulting relabeled residual network remains a lossy network.

Lemma 5.2.2. Each iteration can be performed in $\mathcal{O}(M F(m, n))$ time.

Proof. The bottleneck computations are computing the maximum flow and finding the canonical labels. By Invariant 5.2.1 the relabeled network is a lossy network; thus, the canonical labels can be computed using a Dijkstra shortest path computation.

The next lemma provides a finite bound on the number of iterations.

Lemma 5.2.3. The number of iterations in the primal-dual algorithm is bounded above by the number of different gains of paths in the original network.

Proof. First we show that each excess node's canonical label (other than the sink's) strictly increases after each iteration. After the maximum flow computation and flow update, there are no more augmenting paths in $G_{g, \mu}$ using only unit-gain arcs; otherwise the flow $f$ would not have been maximum. Invariant 5.2.1 guarantees that $G_{g, \mu}$ remains a lossy network. Thus, augmenting paths in the updated network $G_{g, \mu}$ now have gains strictly less than one. This implies that the canonical labels decrease as claimed. The lemma then follows, since a canonical label represents the gain of a path in the original network. 


\subsection{Rounded Primal-Dual}

Algorithm Rounded Primal-Dual (RPD) runs Truemper's primal-dual algorithm in an appropriately rounded network and computes approximately optimal flows.

The input is a lossy network $G$ and error parameter $\xi$. Algorithm RPD first rounds down the gain factors to integer powers of base $b=(1+\xi)^{1 / n}$, as described in Section 4.1. Let $H$ denote the rounded network. Then, RPD runs the primaldual algorithm in the rounded network $H$. Finally, it interprets the resulting flow in the original network. Algorithm RPD is described in Figure 5.2.

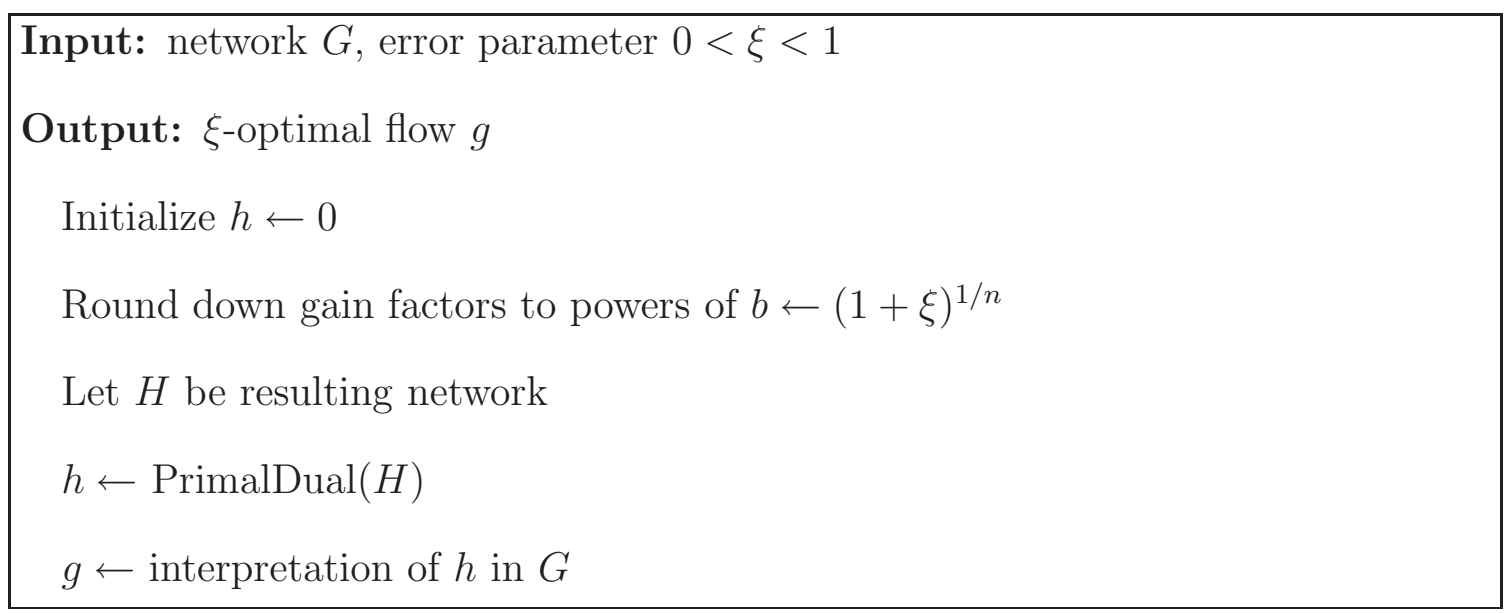

Figure 5.2: Algorithm Rounded Primal-Dual

Theorem 5.3.1. In $\tilde{\mathcal{O}}\left(n^{2} \xi^{-1} \log B\right) \mathrm{MF}(m, n)$ time Algorithm RPD computes a $\xi$ optimal flow in network $G$.

Proof. The gain of a path in network $G$ is between $B^{-n}$ and $B^{n}$. Thus, after rounding to powers of $b$, there are at most $1+\log _{b} B^{2 n}=\mathcal{O}\left(n^{2} \xi^{-1} \log B\right)$ different gains of paths in $H$. By Lemma 5.2.2, each iteration of the primal-dual algorithm requires $\mathcal{O}(M F(m, n))$ time. Thus, by Lemma 5.2.3, RPD finds an optimal flow in 
$H$ in $\tilde{\mathcal{O}}\left(n^{2} \xi^{-1} \log B\right)$ maximum flow computations. The theorem then follows using Corollary 4.1.2.

\subsection{Recursive Rounded Primal-Dual}

Algorithm RPD computes $\xi$-optimal flows in polynomial-time for any constant $\xi>0$. However, it does not compute optimal flows in polynomial time, since the precision required to apply Lemma 2.5.2 is $\xi^{-1}=B^{\Theta m}$. Algorithm Recursive Rounded Primal-Dual (RRPD) is a version of RPD that uses error-scaling, as described in Section 4.2. In order to ensure that the rounding is performed in lossy networks, RPD uses procedure CANCELCYCLES to eliminate flow-generating cycles. It computes $\xi$-optimal flows using $\tilde{\mathcal{O}}\left(n^{2} \log B\right) \log (1 / \xi)$ maximum flow computations and optimal flows with $\tilde{\mathcal{O}}\left(m n^{2} \log ^{2} B\right)$ maximum flow computations.

The input to Algorithm RRPD is a lossy network $G$ and an error parameter $\xi$. In each phase, RRPD computes a 1/2-optimal flow in the relabeled residual network using Algorithm RPD. The input to RPD is required to be a lossy network. So, before each call to RPD, Algorithm RRPD cancels all residual flow-generating cycles using procedure CANCELCyCLES, as described in Section 8.1. Algorithm RRPD is described in Figure 5.3.

Theorem 5.4.1. In $\tilde{\mathcal{O}}\left(n^{2} \log B\right) \operatorname{MF}(m, n) \log (1 / \xi)$ time, Algorithm RRPD computes a $\xi$-optimal flow. In $\tilde{\mathcal{O}}\left(m n^{2} \log ^{2} B\right) \operatorname{MF}(m, n)$ time it can be used to compute an optimal flow. 


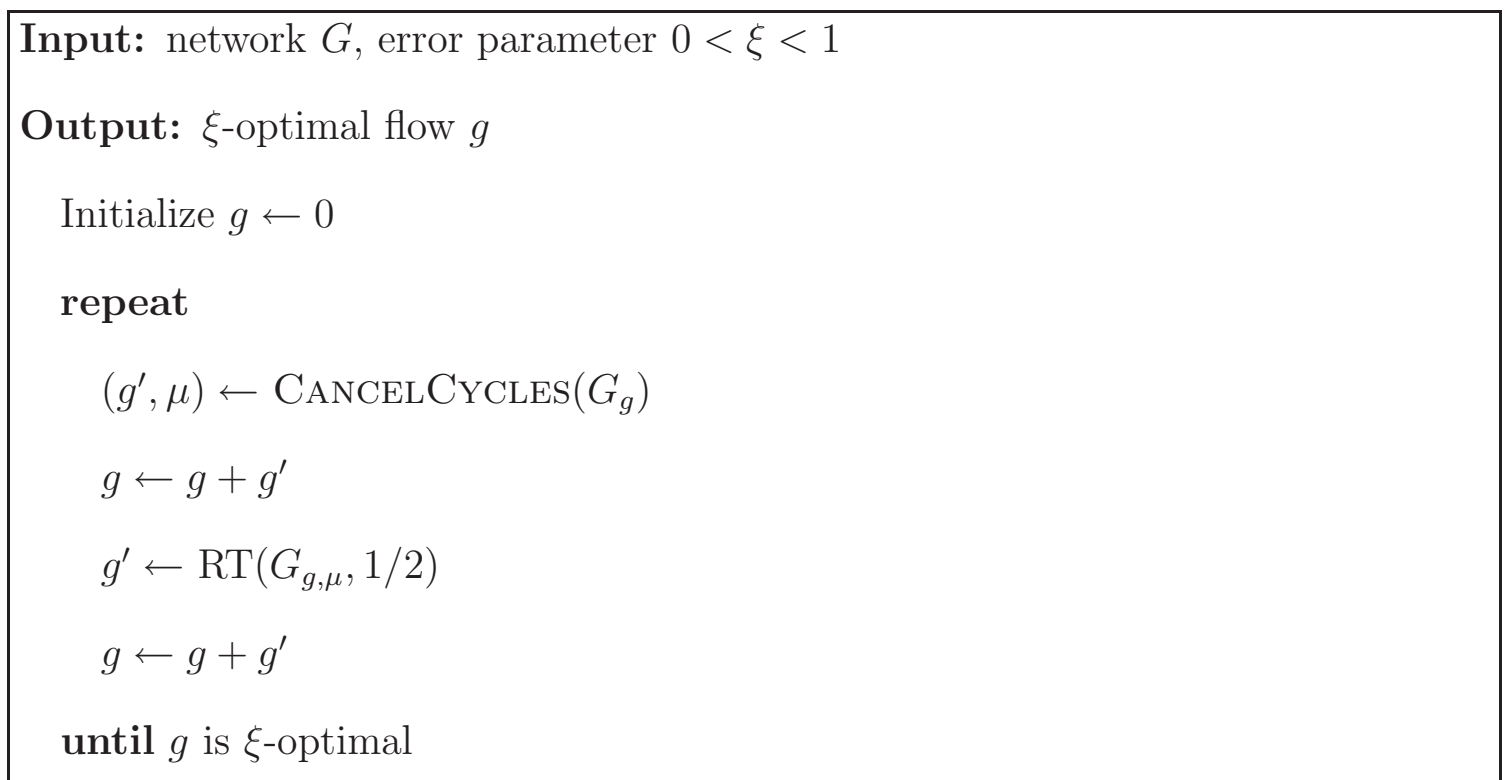

Figure 5.3: Algorithm Recursive Rounded Primal-Dual

Proof. Each phase captures at least half of the remaining flow. Thus, after $\log (1 / \xi)$ phases, the flow is $\xi$-optimal. The bottleneck computation is calling Algorithm RPD; by Theorem 5.3.1, each call requires $\tilde{\mathcal{O}}\left(n^{2} \log B\right)$ maximum flow computations. This says that we compute $\xi$-optimal flows in the stated time bound. We can find an optimal flow by first computing a $B^{-3 m}$-optimal flow, and then applying Lemma 2.5.2 to convert it into an optimal flow.

Using our variant of the CANCELCYCLES procedure from Section 8.2 that only increases node labels by a relatively small amount, we can improve the complexity for computing optimal flows. The best known complexity for the problem among combinatorial algorithms is $\mathcal{O}\left(m^{3} \log B\right)$. For dense networks, our simple primaldual variant already achieves a bound of $\tilde{\mathcal{O}}\left(m^{3} \log B+m^{2} n \log ^{2} B\right)$. 
Theorem 5.4.2. In $\tilde{\mathcal{O}}\left(n^{3} \log B \operatorname{MF}(m, n)+m^{2} n \log ^{2} B\right)$ time RRPD computes an optimal flow.

Proof. As in the proof of Theorem 5.3.1, after each maximum flow computation, the canonical label of (at least) one node decreases by at least $b=(3 / 2)^{1 / n}$. Initially, each label is at most $B^{n}$, and it is never below $B^{-n}$. Assuming the labels never increase, this would give a bound of $n \log _{b} B^{n}=\mathcal{O}\left(n^{3} \log B\right)$ on the number of maximum flow computations.

However, CANCELCyCles might increase some of the node labels. Our variant CANCELCyCles2 cancels all residual flow-generating cycles, but it does not increase any node label substantially. Specifically, Theorem 8.2.6 guarantees that no label increases by more than a factor of $\bar{B}^{3 n}$, where $\bar{B}$ is the biggest gain of a residual arc. We note that the labels that CANCELCYCLES2 returns are not necessarily the canonical labels. However, canonically relabeling the network would only decrease the labels further.

Initially, the input network $G$ is lossy so $\bar{B} \leq 1$. Each call to RPD finds an optimal flow and canonical labels in the rounded network; this implies that no relabeled gain factor exceeds one. Since we round the gains to powers of $b$, there are no residual arcs with relabeled gain factor exceeding $b=(3 / 2)^{1 / n}$ in the unrounded network, i.e., $\bar{B} \leq b$. To find an optimal flow there are $\mathcal{O}(m \log B)$ calls to CANCELCyCLEs2. As a result, throughout the algorithm a single node's label can increase by at most $\mathcal{O}\left(\bar{B}^{m \log B}\right)$. After each maximum flow computation, at least one node's canonical label decreases by a factor of $b$ or more. Thus, an additional $\mathcal{O}(m n \log B)$ maximum 
flow computations and canonical relabelings may be necessary to compensate for the possible increases in node labels.

Canceling flow-generating cycles now becomes a bottleneck operation. Each call requires $\mathcal{O}\left(m n^{2} \log B\right)$ time; it is performed once after every maximum flow computation. 


\section{Chapter 6}

\section{Push-Relabel Method}

In this chapter we adapt the Goldberg-Tarjan [24] push-relabel method for the traditional minimum cost flow problem to the generalized maximum flow problem. Tseng and Bertsekas [56] proposed an $\epsilon$-relaxation method for solving the more general generalized minimum cost flow problem with separable convex costs. However, the complexity of their algorithm is exponential in the input size. We design the first polynomial-time push-relabel method for generalized flows.

First, we review the push-relabel method for the traditional minimum cost flow problem. Then, we give a generalized flow adaption that computes $\xi$-optimal flows in polynomial-time for any constant $\xi>0$. Finally, we show how to find optimal flows in polynomial-time by incorporating error-scaling and canceling flow-generating cycles. We believe our algorithm will be practical and discuss implementation issues. 


\subsection{Push-Relabel Method for Min Cost Flow}

Goldberg and Tarjan $[22,24]$ designed the push-relabel method for the traditional maximum flow and minimum cost flow problems. Push-relabel methods send flow along individual arcs instead of entire augmenting paths. This provides additional flexibility and leads to improvements in both the worst-case complexity as well as practical performance.

Now we describe the push-relabel method for the traditional minimum cost flow problem. The method is divided into cost-scaling phases. In an $\epsilon$-phase, the pushrelabel method maintains a pseudoflow $f$ and node potentials $\pi$ that satisfy the $\epsilon$-complementary slackness conditions:

$$
\forall(v, w) \in G_{f}: \quad c_{\pi}(v, w) \geq-\epsilon .
$$

Within a phase, the algorithm either pushes flow along on arc or increases a node potential. An admissible arc is a residual arc with negative reduced cost. The admissible graph is the subgraph induced by admissible arcs. An active node is a node with positive residual excess. The algorithm repeatedly selects an active node $v$. If there is an admissible arc $(v, w)$ emanating from node $v$, we send $\delta=$ $\min \left\{e_{f}(v), u_{f}(v, w)\right\}$ units of flow from node $v$ to $w$. If $\delta=u_{f}(v, w)$ the push is called saturating; otherwise it is nonsaturating. If there is no such admissible arc, we increase the potential of node $v$ by $\epsilon$. This process is referred to as a relabel operation.

The following lemmas can be found in [1]. The first lemma provides a termination condition and bounds the number of phases. 


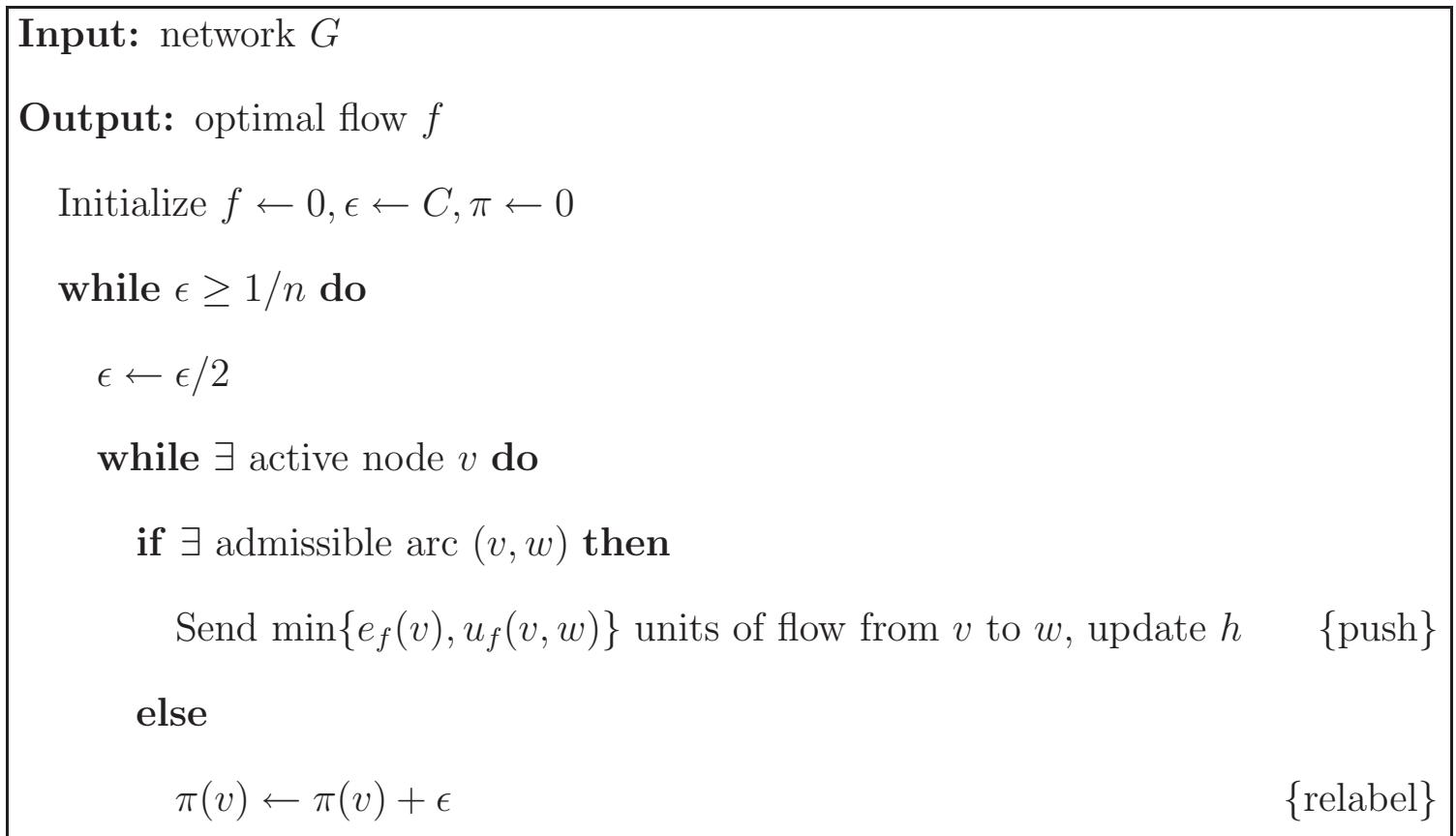

\section{Figure 6.1: Algorithm Push-Relabel}

Lemma 6.1.1. Given a traditional minimum cost flow problem with integer costs between $-C$ and $C$, any pseudoflow is $\epsilon$-optimal for any $\epsilon \geq C$. Moreover, if $\epsilon<1 / n$ then any $\epsilon$-optimal flow is an optimal flow.

Invariant 6.1.2. The algorithm maintains a pseudoflow $f$ and potentials $\pi$ that are $\epsilon$-complementary slack.

The following lemma ensures that flow cannot be sent around a cycle without relabeling.

Invariant 6.1.3. The admissible graph is acyclic during a phase.

The following key lemma limits the number of relabelings. It is then used to bound the number of saturating and nonsaturating pushes operations; these operations cannot be performed too many times without relabelings. 
Lemma 6.1.4. A node potential can increase $\mathcal{O}(n)$ times during a phase.

Between saturating pushes on an arc, both of its endpoints must be relabeled at most once. This leads to the next lemma.

Lemma 6.1.5. There are $\mathcal{O}(m n)$ saturating pushes per phase.

Using a potential function argument, the next lemma bounds the number of nonsaturating pushes of the generic push-relabel algorithm. By choosing the admissible arcs more carefully, it is possible to reduce the bound on the number of nonsaturating pushes to $\mathcal{O}\left(n^{3}\right)$.

Lemma 6.1.6. There are $\mathcal{O}\left(m n^{2}\right)$ nonsaturating pushes per phase.

The bottleneck operation is performing nonsaturating pushes. The (amortized) cost per nonsaturating push can be reduced to $\mathcal{O}(\log n)$ using the dynamic tree data structure developed by Sleator and Tarjan [51]. The main idea is to perform a succession of pushes along a single path in one operation. The dynamic tree data structure maintains a forest of admissible arcs and their residual capacities. Instead of just pushing flow along a single admissible arc, say $(v, w)$, we push flow along $\operatorname{arc}(v, w)$ and then along the tree path from $w$ to its root. Using the dynamic tree data structure, this push operation takes only $\mathcal{O}(\log n)$ (amortized) time instead of possibly $\mathcal{O}(n)$. After a nonsaturating push on arc $(v, w)$, we add it to the forest. It is still admissible, and we know its residual capacity. The results are summarized in the following theorem.

Theorem 6.1.7. The generic push-relabel algorithm requires $\tilde{\mathcal{O}}\left(m n^{2} \log C\right)$ time and $\mathcal{O}(m n \log C)$ time using the dynamic tree data structure. 


\subsection{Push-Relabel for Generalized Flows}

Algorithm Rounded Push-Relabel (RPR) is a generalized flow analog of Goldberg and Tarjan's [24] push-relabel algorithm for the minimum cost flow problem, which is described in Section 6.1. Conceptually, RPR runs the minimum cost flow algorithm with $\operatorname{costs} c=-\log _{b} \gamma$ and fixes an error parameter $\epsilon=\frac{1}{n} \log _{2} b$, where $b=(1+\xi)^{1 / n}$. We define an admissible arc and the admissible graph as before. An active node is a node with positive residual excess and a residual path to the sink. We note that if no such residual path exists and an optimal solution sends flow through this node, then it does not reach the sink. So, it is safe to disregard this useless excess.

Algorithm RPR maintains a flow $h$ and node labels $\mu$. The algorithm repeatedly selects an active node $v$. If there is an admissible $\operatorname{arc}(v, w)$ emanating from node $v$, RPR pushes $\delta=\min \left\{e_{h}(v), u_{h}(v, w)\right\}$ units of flow from node $v$ to $w$. If $\delta=u_{h}(v, w)$ the push is called saturating; otherwise it is nonsaturating. If there is no such admissible arc, RPR increases the label of node $v$ by a factor of $2^{\epsilon}=b^{1 / n}$; this corresponding to an additive potential increase for minimum cost flows. This process is referred to as a relabel operation. Increasing the label of node $v$ can create new admissible arcs emanating from $v$. However, to ensure that we maintain the approximate complementary slackness conditions, we only increase the label by a relatively small amount; this guarantees that we do not create residual flowgenerating cycles. 
The input to Algorithm RPR is a lossy network $G$ and an error parameter $\xi$. Before applying the push-relabel method, RPR rounds the gains to powers of a base $b=(1+\xi)^{1 / n}$, as described in Section 4.1. The method above is then applied to the rounded network $H$. Algorithm RPR is described in Figure 6.2.

Input: lossy network $G$, error parameter $0<\xi<1$
Output: $\xi$-optimal flow $g$

Set base $b=(1+\xi)^{1 / n}$ and round gains in network $G$ to powers of $b$.

Let $H$ be resulting network

Initialize $h \leftarrow 0, \mu \leftarrow 1$

while $\exists$ active node $v$ do

if $\exists$ admissible arc $(v, w)$ then

Send $\min \left\{e_{h}(v), u_{h}(v, w)\right\}$ units of flow on $(v, w)$, update $h$

else

$$
\left.\mu(v) \leftarrow b^{1 / n} \mu(v) \quad \text { \{relabel }\right\}
$$

$g \leftarrow$ interpretation of flow $h$ in $G$

\section{Figure 6.2: Algorithm Rounded Push-Relabel}

We note that our algorithm maintains a generalized flow, i.e., it can have excesses, but no deficits. In contrast, Goldberg and Tarjan's algorithm allows both excesses and deficits. Also their algorithm scales $\epsilon$. We currently do not see how to improve the worst-case complexity by an analogous scaling.

The next two invariants are analogous to Invariants 6.1.2 and 6.1.3.

Invariant 6.2.1. Algorithm RPR maintains a flow $h$ in network $H$ and node labels $\mu$ such that $\gamma_{\mu}(v, w) \leq b^{1 / n}$ for every residual arc $(v, w) \in E_{h}$. 
Proof. We prove by induction on the number of push and relabel operations. Initially all relabeled residual arcs have gain factors at most one. The push operation only sends flow along arcs with relabeled gain above one; therefore only arcs with relabeled gain below one can be added to the residual network. The relabel operation only applies to node $v$ when there are no residual $\operatorname{arcs}(v, w)$ with relabeled gain above one. The label of node $v$ is increased by $b^{1 / n}$. The relabeled gain of outgoing residual arcs can increase to at most $b^{1 / n}$. The relabeled gain of incoming arcs decreases. The relabeled gain of other arcs do not change.

The next invariant relies on the fact that the network is rounded. It is needed to ensure that the algorithm finds an optimal flow in the rounded network $H$.

Invariant 6.2.2. During Algorithm RPR, the admissible graph is acyclic.

Proof. We prove by induction on the number of push and relabel operations. Initially there are no admissible arcs, so the admissible graph is acyclic. A push operation sends flow along an arc with relabeled gain exceeding one. The reversal of this arc may be added to the residual network, but it is not admissible, as its relabeled gain factor is less than one. Thus, pushes do not create admissible arcs, so the admissible graph remains acyclic. The relabel operation increases the label of some node, say $v$, by $b^{1 / n}$. This might create admissible arcs leaving $v$. All arcs entering $v$ have their relabeled gain decreased by $b^{1 / n}$. By Invariant 6.2.1, these arcs now have relabeled gains at most one, and hence are inadmissible. Consequently, the relabel operation does not create any directed admissible cycle passing through node $v$. 
Invariant 6.2.3. Algorithm $R P R$ maintains a flow $h$ in network $H$ such that $H_{h}$ has no residual flow-generating cycles.

Proof. Let $\Gamma$ be a residual cycle with $\gamma(\Gamma) \geq 1$. By Invariant 6.2.1 all relabeled gains in $\Gamma$ are at most $b^{1 / n}$. By Invariant 6.2.2, not all of the arcs in $\Gamma$ have relabeled gain above one. Thus $\gamma_{\mu}(\Gamma) \leq b^{1-1 / n}<b$. Then $\gamma(\Gamma) \leq 1$ since $\gamma(\Gamma)=\gamma_{\mu}(\Gamma)$ and all gains are powers of $b$.

Lemma 6.2.4. Upon terminates, Algorithm RPR outputs a $\xi$-optimal flow $g$ in network $G$.

Proof. Invariant 6.2.3 and Theorem 2.3.7 imply that if the algorithm terminates then the flow $h$ is optimal in network $H$, since then there are no active nodes or residual flow-generating cycles in $H_{h}$. By Corollary 4.1.2 the flow $g$ is $\xi$-optimal in $G$.

The following key lemma bounds the number of label increases. This yields a good bound because by rounding the network, we were able to choose a large enough base $b$.

Lemma 6.2.5. There are $\mathcal{O}\left(n^{3} \xi^{-1} \log B\right)$ relabels per node.

Proof. We show that the label of an active node cannot get too big. This limits the number of relabelings. Let $v$ be an active node. Let $P$ be a $v$ - $t$ path in $H_{h, \mu}$. By Invariant 6.2.1 $\gamma_{\mu}(P) \leq b$. By definition, $\gamma_{\mu}(P)=\mu(v) \gamma(P)$ and $\gamma(P) \geq B^{-n}$. Thus, $\mu(v) \leq b B^{n}$. Since each relabeling of node $v$ increases its label by a factor of 
$b^{1 / n}$, the number of times a node is relabeled is at most

$$
\log _{b^{1 / n}}\left(b B^{n}\right)=n+\frac{n^{2} \log B}{\log b}=\mathcal{O}\left(n^{3} \xi^{-1} \log B\right) .
$$

As for the minimum cost flow problem, once we have a bound on the number of relabeling operations, we can bound the number of saturating and nonsaturating pushes.

Lemma 6.2.6. There are a total of $\mathcal{O}\left(m n^{3} \xi^{-1} \log B\right)$ saturating pushes.

Proof. We show below that between two consecutive saturating pushes on $\operatorname{arc}(v, w)$, node $v$ and node $w$ must each be relabeled at least once. Combining this fact with Lemma 6.2.5 implies that each arc is saturated at most $\mathcal{O}\left(n^{3} \xi^{-1} \log B\right)$ times.

Consider a saturating push on arc $(v, w)$. At the time of the push, $\gamma_{\mu}(v, w)>1$, since $(v, w)$ is admissible. Before arc $(v, w)$ can be saturated again, flow must be pushed back along the reverse arc $(w, v)$. At this time, $\gamma_{\mu}(w, v)>1$, or equivalently $\gamma_{\mu}(v, w)<1$. So, between the original saturating push and the reverse push, node $w$ must have been relabeled at least once. At the time of the subsequent saturating push on $\operatorname{arc}(v, w), \gamma_{\mu}(v, w)>1$. Before this can happen, node $v$ must have been relabeled at least once.

Lemma 6.2.7. There are a total of $\mathcal{O}\left(m n^{4} \xi^{-1} \log B\right)$ nonsaturating pushes.

Proof. We prove the lemma using the potential function $\Phi=\sum_{v \text { is active }} n(v)$, where $n(v)$ is the number of nodes reachable from $v$ (including itself) in the admissible 
graph. At the beginning of the algorithm $\Phi \leq n$, since initially there are no admissible arcs. We now consider the effects of relabeling, saturating, and nonsaturating push operations on the potential function.

After a saturating push on arc $(v, w)$, node $w$ might become active. This can increase $\Phi$ by at most $n(w) \leq n$. Lemma 6.2 .6 implies that the total increase due to saturating pushes is $\mathcal{O}\left(m n^{4} \xi^{-1} \log B\right)$.

Relabeling node $v$ can create new admissible arcs leaving $v$, so $n(v)$ can increase by as much as $n$ units. However, as in the proof of Invariant 6.2.2, after the relabeling, all arcs entering $v$ are not admissible; thus $n(w)$ does not increase for any other node $w$. By Lemma 6.2.5, the total increase in $\Phi$ due to relabelings is $\mathcal{O}\left(n^{4} \xi^{-1} \log B\right)$.

After a nonsaturating push on arc $(v, w)$, we show that $\Phi$ decreases by at least one unit. Since the push is nonsaturating, node $v$ becomes inactive, and node $w$ might become active. The push decreases $\Phi$ by $n(v)$ and might increase it by $n(w)$. Note that $n(v) \geq n(w)+1$, since every node reachable in the admissible graph from $w$ is also reachable from $v$; moreover $v$ is not reachable from $w$ since Invariant 6.2.2 guarantees that the admissible graph is acyclic. Thus, $\Phi$ decreases by at least one unit.

The potential function is initially at most $n$. It increases by $\mathcal{O}\left(m n^{4} \xi^{-1} \log B\right)$ from saturating pushes and relabelings. Each nonsaturating push decreases the potential function by at least one. The lemma follows, since $\Phi$ cannot become negative. 
If we implement the algorithm exactly as stated, it turns out that the bottleneck computation is finding active nodes, since it requires $\mathcal{O}(m)$ time to determine which nodes can reach the sink. Instead, we could only need recompute active nodes before each relabeling operations. Between recomputations, we act as though all excess nodes created are active. The proof of correctness remains valid even if RPR relabels or pushes flow from inactive excess nodes. As noted earlier, nodes that cannot reach the sink can be disregarded for the remainder of the algorithm. Thus, the number of relabeling operations can be bounded as before; the bounds on the number of pushes do not change either.

The real bottleneck computation is performing nonsaturating pushes. As for the traditional minimum cost flow problem, the (amortized) time per nonsaturating push can be reduced to $\mathcal{O}(\log n)$ using the dynamic tree data structure. One might be worried that the dynamic tree data structure can not be used directly in generalized networks, since it does not account for the gain factors. Fortunately, all admissible arcs have relabeled gain factor above one. We choose to push the same quantity of flow through every arc in the path. This creates additional node excesses, but no deficits. Our analysis is not affected by the creation of additional excess. This leads to the following theorem.

Theorem 6.2.8. In $\tilde{\mathcal{O}}\left(m n^{3} \xi^{-1} \log B\right)$ time Algorithm RPR computes a $\xi$-optimal flow in network $G$. 


\subsection{Recursive Rounded Push-Relabel}

Algorithm RPR computes $\xi$-optimal flows in $\tilde{\mathcal{O}}\left(m n^{3} \xi^{-1} \log B\right)$ time. However, it does not compute optimal flows in polynomial time since the precision required to apply Lemma 2.5.2 is $\xi^{-1}=B^{\mathcal{O}(m)}$. Algorithm Recursive Rounded Preflow-Push (RRPR) is a version of RPR that also incorporates error-scaling, as described in Section 4.2. To ensure that the rounding is performed in lossy networks, RRPR uses CancelCycles to eliminate flow-generating cycles. It computes $\xi$-optimal flows in $\tilde{\mathcal{O}}\left(m n^{3} \log B\right) \log (1 / \xi)$ time and optimal flows in $\tilde{\mathcal{O}}\left(m^{2} n^{3} \log ^{2} B\right)$ time.

The input to RRPR is a network $G$ and an error parameter $\xi$. In each phase, RRPR computes a 1/2-optimal flow in the relabeled residual network using Algorithm RPR. The input to RPR is required to be a lossy network, so before each call to RPR, Algorithm RRPR cancels all residual flow-generating cycles using procedure CancelCycles, as described in Section 8.1. Algorithm RRPR is described in Figure 6.3.

Theorem 6.3.1. Algorithm RRPR computes a $\xi$-optimal flow in network $G$ in $\tilde{\mathcal{O}}\left(m n^{3} \log B\right) \log (1 / \xi)$ time. It computes an optimal flow in $\tilde{\mathcal{O}}\left(m^{2} n^{3} \log ^{2} B\right)$ time.

Proof. Each phase captures at least half of the remaining flow. Thus, after $\log (1 / \xi)$ phases, the flow is $\xi$-optimal. The bottleneck computation is calling Algorithm RPR; by Theorem 6.2.8, each call requires $\tilde{\mathcal{O}}\left(m n^{3} \log B\right)$ time. This says that we compute $\xi$-optimal flows in the stated time bound. We can find an optimal flow in the stated time bound by first computing a $B^{-3 m}$-optimal flow and then using Lemma 2.5.2 to convert it into an optimal flow. 


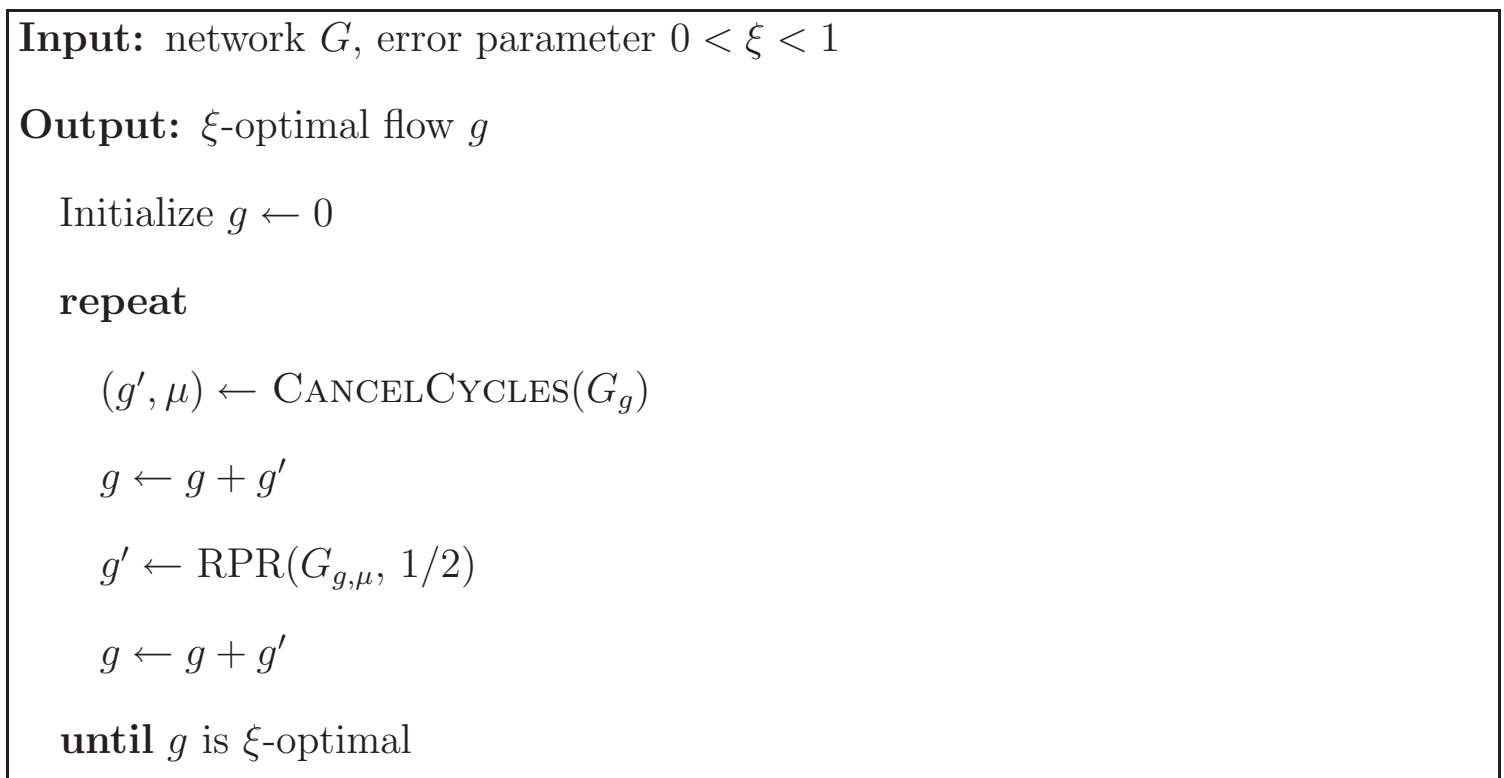

Figure 6.3: Algorithm Recursive Rounded Push-Relabel

\subsection{Issues for a Practical Implementation}

The push-relabel algorithm for the traditional maximum flow problem is currently believed to be the most practical. We believe our generalized push-relabel algorithm will also be practical. In some preliminary computational experiments, we implemented a version of RRPR. The overwhelming bottleneck computation was canceling flow-generating cycles. To avoid this bottleneck, we also implemented a version of the RPR approximation scheme. We plan to investigate how the algorithm's performance will deteriorate as we require improved precision. Also, if we require an optimal solution, we can determine an error parameter for which the RPR algorithm is fast, and then use the resulting solution as a "warm start" for a generalized network simplex algorithm. We plan to perform computational experiments using data for the machine scheduling problem discussed in Section 1.3. 


\section{Chapter 7}

\section{Fat-Path}

In this chapter we present a new Fat-Path variant for the generalized maximum flow problem. It matches the best known complexity bound for computing approximate flows, and it is much simpler than Radzik's variant. First, we review the details of the original Fat-Path capacity-scaling algorithm of Goldberg, Plotkin and Tardos [20]. Next, we describe Radzik's [49] Fat-Path variant. Finally, we present our new Fat-Path variant.

\subsection{Most-Improving Augmenting Path}

In this section we describe a simple (but not the most efficient) version of the Fat-Path capacity-scaling algorithm of Goldberg, Plotkin, and Tardos [20]. The most-improving augmenting path algorithm [52] generalizes the maximum capacity

augmenting path algorithm for the traditional maximum flow problem, e.g. see [1] . 
This version can also be used to generate an initial flow whose value is within a factor of $m$ of the optimum in $\tilde{\mathcal{O}}(m)$ time.

The value of an augmenting path is the maximum amount of flow that can reach the sink, while respecting the capacity limits, by sending excess from the first node of the path to the sink. A most-improving augmenting path is an augmenting path with the highest value. The algorithm repeatedly sends flow along mostimproving augmenting paths. Since these may not be highest gain augmenting paths, this may creates residual flow-generating cycles. After each augmentation, the algorithm cancels all residual flow-generating cycles, so that computing the next most-improving path can be done efficiently. Intuitively, canceling flow-generating cycles can be interpreted as rerouting flow from its current paths to highest-gain paths, but not all of the rerouted flow reaches the sink.

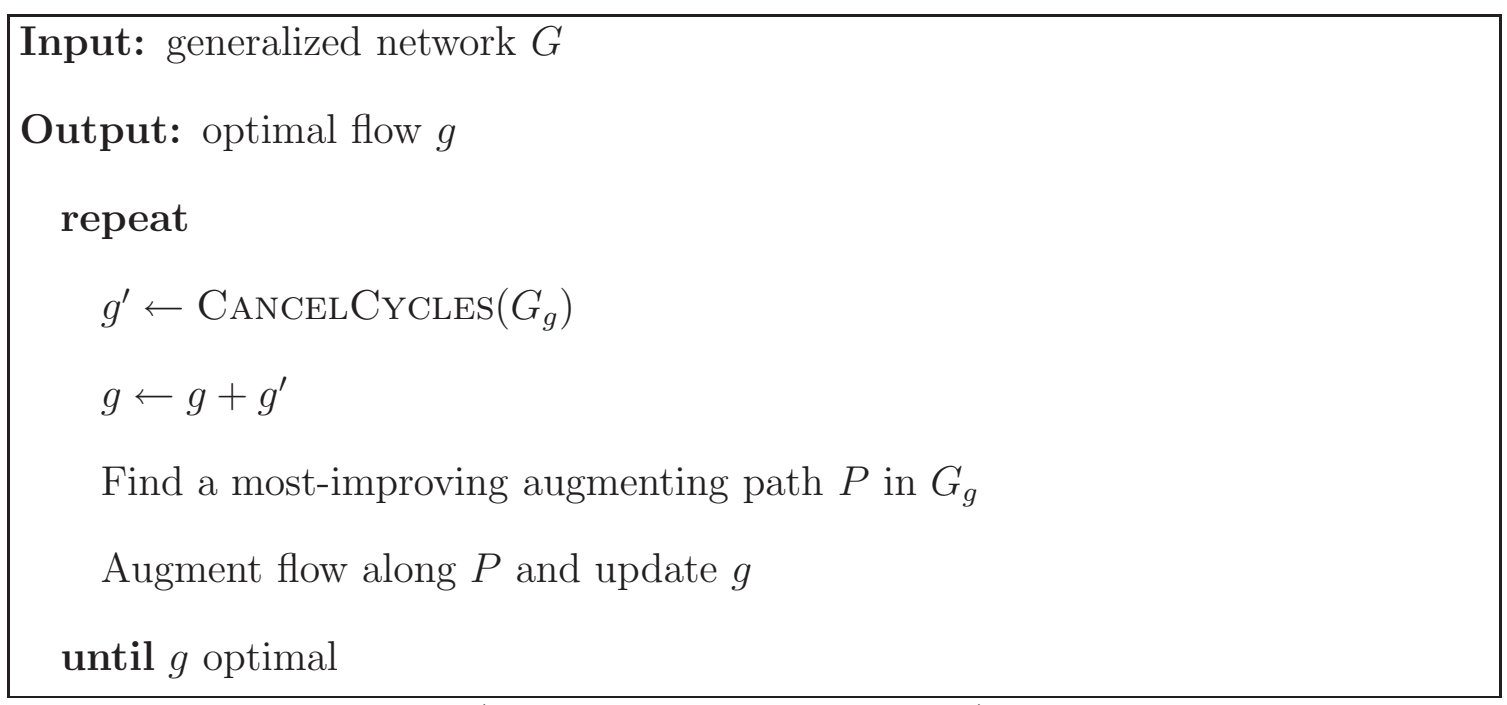

Figure 7.1: Algorithm Most-Improving Augmenting Path

Lemma 7.1.1. In $\mathcal{O}(m \log (1 / \xi))$ iterations, the most-improving augmenting path algorithm computes a $\xi$-optimal flow. 
Proof. By Corollary 2.3.4, the optimal flow in a lossy network can be decomposed into at most $m$ augmenting paths. The algorithm selects the path that generates the maximum amount of excess at the sink. Thus, each iteration captures at least a $1 / m$-fraction of the remaining flow. Since $(1-1 / m)^{m} \approx 1 / e$, the algorithm captures at least half of the remaining flow after $\mathcal{O}(m)$ iterations, and the lemma follows.

We give a new and faster subroutine for computing a most-improving augmenting path in a lossy network. Our subroutine is based on Dijkstra's shortest path algorithm. For each node $v$, the algorithm maintains a label $d(v)$. Upon termination of the algorithm, $d(v)$ is the maximum amount of flow arriving at $v$ that can be sent from the source along a path; during the algorithm $d(v)$ is always a lower bound on this quantity. Our algorithm is identical to Dijkstra's shortest path algorithm, except in the way the labels are updated. The algorithm selects a new node $v$ with the largest value of $d(v)$. Assuming $d(v)$ represents the maximum amount of flow we can send to $v$, then we could send it to node $w$ along arc $(v, w)$. The amount that reaches $w$ is $\gamma(v, w) \times \min \{d(v), u(v, w)\}$. This leads to updating $d(w)$ with $\max \{\gamma(v, w) \times \min \{d(v), u(v, w)\}, d(w)\}$ for all $(v, w) \in E$. The algorithm is described in Figure 7.2.

The following invariant establishes the correctness of the procedure and can be proved by induction. The proof is straightforward and analogous to Dijkstra's.

Invariant 7.1.2. At any point during the algorithm, the label of each node in $S$ is optimal. Moreover, the label of each node in $\bar{S}$ is the maximum amount of flow that can reach that node, provided that each internal node of the path lies in $S$. 
Input: lossy network $G$

Output: $d(v)=$ maximum amount of flow that can reach $v$ along a path

Initialize $\forall v \in V: d(v) \leftarrow e(v), S=\{v: d(v)>0\}$

while $\bar{S} \neq \emptyset$ do

$$
\begin{aligned}
& v \leftarrow \operatorname{argmax}\{d(i): i \in \bar{S}\} \\
& S \leftarrow S \cup\{v\} \\
& \text { for all }(v, w) \in E \text { do } \\
& \qquad \operatorname{cap}=\gamma(v, w) \times \min \{d(v), u(v, w)\} \\
& \quad \text { if } d(w)<\operatorname{cap} \text { then } \\
& \qquad d(w) \leftarrow \operatorname{cap}, \operatorname{pred}(w) \leftarrow v
\end{aligned}
$$

Figure 7.2: Subroutine Finding a Most-Improving Augmenting Path

A straightforward implementation using Fibonacci heaps, as in the FredmanTarjan [17] implementation of Dijkstra's algorithm, implies the following theorem.

Theorem 7.1.3. There exists a $\mathcal{O}(m+n \log n)$ time algorithm to find a mostimproving path in a lossy network.

Actually, subsequent to this thesis, Wayne discovered that the same ideas can be used to find a most-improving generalized augmenting path. However, the computation is more complicated. Essentially, he replaces our Dijkstra variant with an analogous variant of a two variable per inequality feasibility detector. This is the first known purely "augmenting path" style algorithm for the problem that is polynomial. It repeatedly sends flow only along augmenting paths and GAPs. 
Finding a Good Initial Flow. Our approximation algorithms for the generalized maximum flow problem need reasonably good estimates of the optimum value; otherwise they may take as long as the exact algorithms. Radzik [49] proposed an $\mathcal{O}(m) S P(m, n)$ time greedy augmentation algorithm that finds a flow whose value is within a factor of $n$ of the optimum. Alternatively, we can find a flow whose value is within a factor $m$ of the optimum in $\mathcal{O}(m+n \log n)$ time. By Corollary 2.3.4, a most-improving augmenting path gives such a flow.

Corollary 7.1.4. In $\mathcal{O}(m+n \log n)$ time we can determine an initial parameter $\Delta_{0}$ which satisfies $\mathrm{OPT}(G) \leq \Delta_{0} \leq m \mathrm{OPT}(G)$

Remark 7.1.5. In contrast, for the minimum cost flow problem, it is NP-hard to find a most-improving cycle. Although many minimum cost flow algorithms have generalized flow analogs, it is very unlikely that finding a most-improving augmenting path will have a proper analog for the minimum cost flow problem. However, by solving a sequence of assignment problems, Baharona and Tardos [4] showed how to efficiently find a collection of cycles whose value is at least as good as the most-improving cycle.

\subsection{Fat-Path}

Now, we review the original Fat-Path algorithm and analysis of Goldberg, Plotkin, and Tardos [20]. The bottleneck computation in the most-improving augmenting path algorithm is canceling flow-generating cycles, which is done after each augmentation. The main idea of the Fat-Path algorithm is to perform many augmentations, 
before cycle-canceling. The Fat-Path algorithm can be viewed as a generalized flow analog of Orlin's [45] capacity-scaling minimum cost flow algorithm, which, in turn, is a variant of Edmonds and Karp's [13] algorithm. The underlying idea is to send excess to the sink along augmenting paths with sufficiently large capacity. A $\delta$-fat path is a residual path that has enough capacity to increase the excess at the sink by at least $\delta$ units of flow, given sufficient excess at the first node of the path. The Fat-Path algorithm does not necessarily send flow along overall highest gain augmenting paths. So, periodically it cancels all residual flow-generating cycles, so that computing subsequent fat-paths can be done efficiently.

The input to the Fat-Path algorithm is a network $G$ and error parameter $\xi$. The Fat-Path algorithm solves the generalized maximum flow in phases. A phase is characterized by a scaling parameter $\Delta$, which provides an upper bound on the excess discrepancy, i.e., the difference between the value of the current flow and the value of the optimal flow. We initialize $\Delta$, as in Corollary 7.1.4, so that $\mathrm{OPT}(G) \leq \Delta \leq m \mathrm{OPT}(G)$. Each phase decreases $\Delta$ by a factor of two. When $\Delta$ is sufficiently small, we will show that we have an approximately optimal flow. Each phase consists of two components: canceling all residual flow-generating cycles and finding fat-paths. At the beginning of a phase, all residual flow-generating cycles are canceled, using procedure CANCELCyCLES, as described in Section 8.1. Next, procedure FatAugmentations, which we describe in Section 7.3, is used to repeatedly augment flow along $\delta$-fat paths, where $\delta=\Delta /(2 m)$, until no such paths remain. At this point, $\Delta$ is decreased by a factor of two, and a new phase begins. We describe the Fat-Path algorithm in Figure 7.3. 


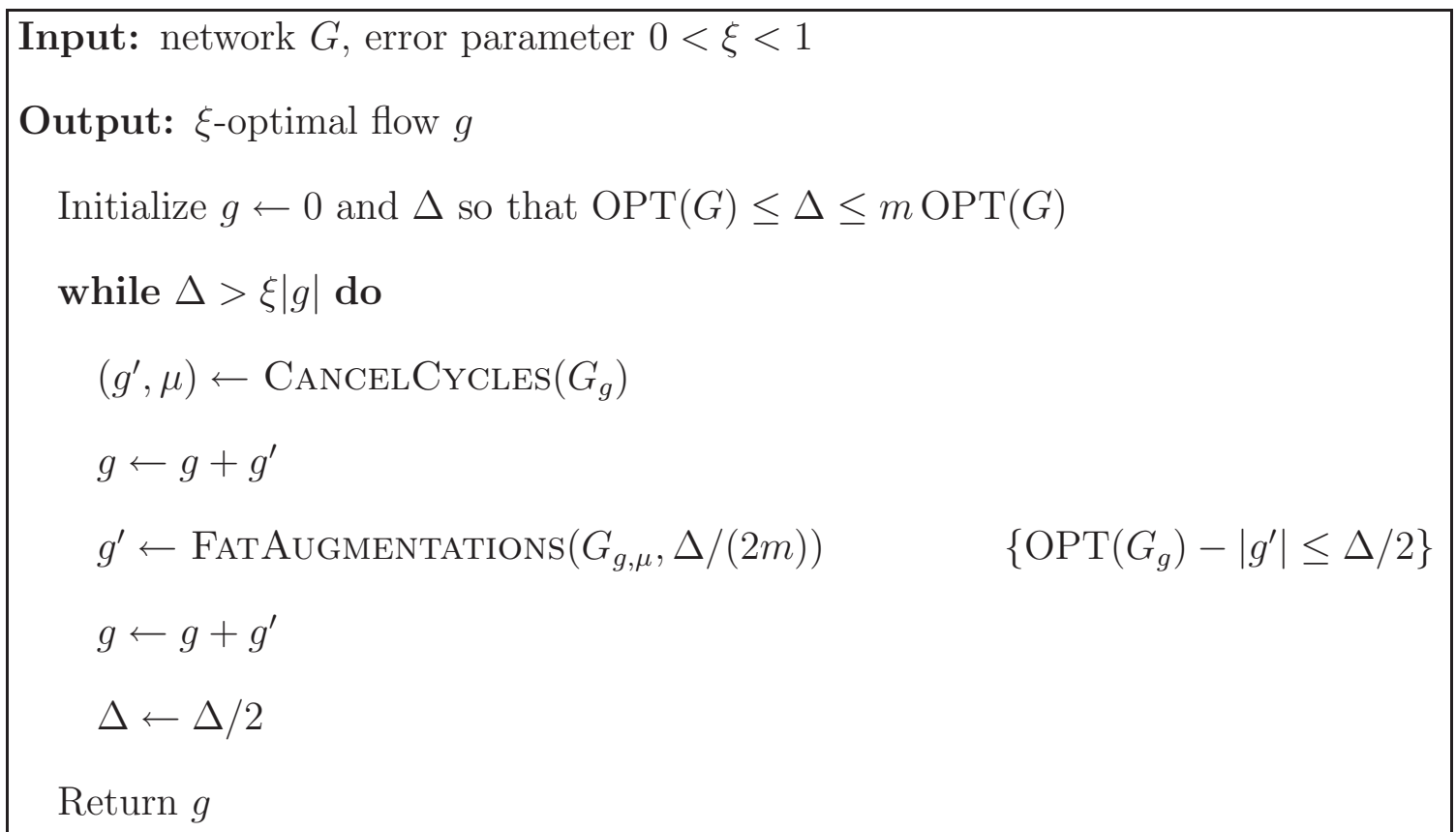

Figure 7.3: Algorithm Fat-Path

Procedure FatAugmentations is the core of the Fat-Path algorithm. It efficiently determines a flow with relatively small excess discrepancy. The following lemma summarizes the result of procedure FATAugmentations. We defer its proof until Section 7.3. Once we establish this key lemma, the rest of the analysis is straightforward.

Lemma 7.2.1. If $G_{g, \mu}$ is a lossy network, then in $\mathcal{O}\left((m+n \log n)\left(n+\mathrm{OPT}\left(G_{g}\right) / \delta\right)\right)$ time, procedure FatAugmentations $\left(G_{g, \mu}, \delta\right)$ outputs a flow $g^{\prime}$ of value at least $\operatorname{OPT}\left(G_{g}\right)-m \delta$

The following invariant says that $\Delta$ acts as a good proxy for the excess discrepancy, i.e., when $\Delta$ is small then so is the excess discrepancy. 
Invariant 7.2.2. The parameter $\Delta$ remains an upper bound on the excess discrepancy, i.e., $\operatorname{OPT}(G)-|g| \leq \Delta$.

Proof. We initialize $g=0$ and $\mathrm{OPT}(G) \leq \Delta$. The excess discrepancy can only decrease throughout the algorithm, so we only need to worry about what happens when $\Delta$ is decreased. By Lemma 7.2.1, FAtAugmentations returns a flow $g^{\prime}$ in $G_{g}$ whose value is at least $\mathrm{OPT}\left(G_{g}\right)-m \delta=\mathrm{OPT}\left(G_{g}\right)-\Delta / 2$. Thus $g+g^{\prime}$ has excess discrepancy at most $\Delta / 2$, so when $\Delta$ is halved, it remains an upper bound on the excess discrepancy.

The next guarantees that upon termination the flow is of the desired quality.

Lemma 7.2.3. Upon termination, the flow $g$ is $\xi$-optimal.

Proof. Upon termination, $\Delta \leq \xi|g| \leq \xi \mathrm{OPT}(G)$. By Invariant 7.2.2 we also have $\operatorname{OPT}(G)-|g| \leq \Delta$. The lemma combines these two inequalities.

The next lemma bounds the number of phases.

Lemma 7.2.4. There are $\mathcal{O}(\log (m / \xi))$ phases.

Proof. Initially $\Delta \leq m \mathrm{OPT}(G)$. We show that the algorithm will terminate before $\Delta \leq \xi \mathrm{OPT}(G) / 2$. The lemma then follows, since $\Delta$ halves in each phase. If $\Delta \leq \xi \mathrm{OPT}(G) / 2$, then we have

$$
2 \Delta \leq \xi \mathrm{OPT}(G) \leq \xi \Delta+\xi|g| \leq \Delta+\xi|g|
$$

where the second inequality follows from Invariant 7.2.2. This implies that $\Delta \leq \xi|g|$, which is precisely the stopping condition. 
Theorem 7.2.5. In $\tilde{\mathcal{O}}\left(m n^{2} \log B\right) \log (1 / \xi)$ time The Fat-Path algorithm computes a $\xi$-optimal flow.

Proof. Lemma 7.2.3 and Theorem 4.1.1 ensure the flow returned is of the desired quality. By Lemma 7.2.4 there are $\mathcal{O}(\log (m / \xi))$ phases. The complexity of each phase is dominated by procedures CANCELCyCLES and FATAugmentaTions. By Theorem 8.1.10, CAnCELCyCles requires $\mathcal{O}\left(m n^{2} \log n \log B\right)$ time per phase. FatAugmentations requires $\mathcal{O}(m(m+n \log n))$ time per phase; this follows from Lemma 7.2.1, since Invariant 7.2.2 guarantees that the algorithm maintains $\Delta \geq \operatorname{OPT}\left(G_{g}\right)$ and we choose $\delta=\Delta /(2 m)$.

\subsection{Fat Augmentations}

In this section, we review the FATAugmentations procedure of Goldberg, Plotkin, and Tardos [20]; it is the core of their Fat-Path algorithm. The purpose of this procedure is to repeatedly augment flow along $\delta$-fat paths, until no such paths remain. At this point, we can show that the excess discrepancy is relatively small. The procedure is based on Dijkstra's shortest path algorithm.

Definitions and Intuition. A highest-gain $\delta$-fat path is a highest gain path, among all $\delta$-fat paths. The FATAugmentations procedure repeatedly augments flow along highest-gain $\delta$-fat paths. By only selecting such paths, FATAUGmentATIONS is able to find subsequent $\delta$-fat paths efficiently. 
A $\delta$-fat arc is an arc that participates in some $\delta$-fat path. Consider a highestgain augmenting path from some node to the sink. Either this path is $\delta$-fat or there is (at least) one bottleneck arc, say $(v, w)$, that would be saturated when flow is augmented along this path. The capacity of this bottleneck arc $(v, w)$ times the gain of the part of the path from $v$ to the sink, say $P_{1}$, is less than $\delta$, i.e., $u(v, w) \gamma\left(P_{1}\right)<\delta$. In this case we say that $(v, w)$ is a critical arc. Critical arcs cannot be $\delta$-fat. The main idea of the FAtAugmentations subroutine is to disregard critical arcs, while constructing a highest-gain $\delta$-fat path.

Let $G_{g}^{\delta}$ denote the subgraph induced by residual $\delta$-fat arcs. To improve the efficiency for repeatedly finding highest-gain $\delta$-fat paths, FATAuGMEnTATions maintains node labels $\mu$ so that $G_{g, \mu}^{\delta}$ is a lossy network. This guarantees that there are no flow-generating cycles in $G_{g}^{\delta}$, and allows us to use a Dijkstra style computation instead of a Bellman-Ford style one. Note that non $\delta$-fat arcs may have relabeled gain above one. After sending flow along a highest-gain $\delta$-fat path, we will show that disregarded arcs do not become $\delta$-fat. This fact will ensure that $G_{g, \mu}^{\delta}$ remains a lossy network after augmenting flow along a highest-gain $\delta$-fat path.

The Procedure. The input to FatAugmentations is a lossy network $G$ and fatness parameter $\delta$. The output is a flow $g$ such that $G_{g, \mu}^{\delta}$ is a lossy network with no augmenting paths. Analogous to Dijkstra's algorithm, the procedure constructs not just a single highest-gain $\delta$-fat path, but a highest gain $\delta$-fat tree, i.e., a tree rooted at the sink such that the tree path from any node to the sink is $\delta$-fat, and has the highest gain among all such paths. A highest-gain $\delta$-fat tree corresponds 
to a shortest path tree in $G_{g, \mu}^{\delta}$, using $\operatorname{costs} c=-\log \gamma$. Since $G_{g, \mu}^{\delta}$ is a lossy network all arc costs are nonnegative and we construct the tree with a Dijkstra style computation. The difficulty is that we do not know which arcs are $\delta$-fat ahead of time, i.e. we do not know the network $G_{g, \mu}^{\delta}$ on which we wish to apply Dijkstra's algorithm! Surprisingly, FAtAugmentations is able to simultaneously disregard non $\delta$-fat while running Dijkstra's algorithm on the proper network.

For each node $v \in V$, we denote the maximum gain of the $\delta$-fat $v$ - $t$ path found so far by node label Gain $(v)$. As in Dijkstra's algorithm, at each iteration we find a node $v$ that has the largest Gain $(v)$ among non-tree nodes and update the Gain of its neighbors:

$\forall u$ such that $(u, v) \in E_{g}: \quad \operatorname{Gain}(u) \leftarrow \max \left\{\operatorname{Gain}(u), \operatorname{Gain}(v) \gamma_{\mu}(u, v)\right\}$

However, we should only perform the Dijkstra update for $\delta$-fat arcs, as we build the tree. Before performing the Dijkstra update, we disregard an arc if

$$
u_{g, \mu}(u, v) \gamma_{\mu}(u, v) \operatorname{Gain}(v)<\delta .
$$

We will show that an arc is disregarded if and only if it is not $\delta$-fat.

After the tree is constructed, we multiply the label of node $v$ by $\operatorname{Gain}(v)$, so that every tree arc has unit relabeled gain. Then we find a node in the tree with positive excess, and augment flow along the $\delta$-fat tree path. We will show that $G_{g, \mu}^{\delta}$ remains a lossy network. The method is then repeated until no such $\delta$-fat paths remain. Procedure FatAugmentations is described in Figure 7.4. 
Input: lossy network $G$, fatness parameter $\delta$

Output: flow $g$, labels $\mu$ such that $G_{g, \mu}^{\delta}$ is a lossy network with no augmenting paths

Initialize $g \leftarrow 0, \mu \leftarrow 1$

repeat

Initialize $\operatorname{Gain}(v) \leftarrow 0$ for all $v \neq t, \operatorname{Gain}(t) \leftarrow 1, T \leftarrow \emptyset$

while there are nodes not in $T$ with nonzero Gain do

$v \leftarrow$ node not in $T$ with highest Gain

add $\operatorname{arc}(v, \operatorname{parent}(v))$ to $T$

for all nodes $u$ such that $(u, v) \in E_{g}$ do

if $u_{g, \mu}(u, v) \gamma_{\mu}(u, v) \operatorname{Gain}(v)<\delta$ then

disregard $\operatorname{arc}(u, v)$

else

if $\operatorname{Gain}(u)<\operatorname{Gain}(v) \gamma_{\mu}(u, v)$ then

$\operatorname{Gain}(u) \leftarrow \operatorname{Gain}(v) \gamma_{\mu}(u, v), \operatorname{parent}(u) \leftarrow v$

Update $\mu(v) \leftarrow \mu(v) \times \operatorname{Gain}(v)$

If there exists augmenting path in $T$, then send flow along path and update $g$ until $G_{g}$ has no $\delta$-fat paths

Figure 7.4: Subroutine Fat Augmentations 
The following lemma says that in each while loop, FatAugmentations constructs a highest-gain $\delta$-fat tree. It assumes that $G_{g, \mu}^{\delta}$ is a lossy network. Invariant 7.3.2 below guarantees that $G_{g, m u}^{\delta}$ remains a lossy network during the procedure.

Lemma 7.3.1. If $G_{g, \mu}^{\delta}$ is a lossy network at the beginning of the while loop, then it constructs a highest-gain $\delta$-fat tree.

Proof. We show below that an arc is disregarded if and only if it is not $\delta$-fat. Since $G_{g, \mu}^{\delta}$ is a lossy network, all $\delta$-fat arcs have nonnegative length using lengths $-\log \gamma_{\mu}$. Then, the while loop is an implementation of Dijkstra's algorithm on the subgraph induced by $\delta$-fat arcs. Consequently, it constructs a highest-gain $\delta$-fat tree.

Now, we show that an arc is disregarded if and only if it is not $\delta$-fat. We prove by induction on the number of arcs examined. Let $(u, v)$ denote the arc currently being examined. By induction, all previous arcs were disregarded if and only if they were not $\delta$-fat. Thus, the tree constructed so far is a highest-gain $\delta$-fat subtree.

First, we consider the case when $(u, v)$ is not disregarded. We show that $(u, v)$ is $\delta$-fat. Since $(u, v)$ is not disregarded, we have

$$
u_{g, \mu}(u, v) \gamma_{\mu}(u, v) \operatorname{Gain}(v) \geq \delta .
$$

Let $P$ be the $v$ - $t$ path in the current highest-gain $\delta$-fat subtree. Inductively, $P$ is a $\delta$-fat path. Thus, $(u, v)$ and $P$ form a $\delta$-fat path also, since $\operatorname{Gain}(v)=\gamma(P)$.

Now, we consider the case when $(u, v)$ is a $\delta$-fat arc. By definition, there exists a $v$ - $t$ path $P_{1}$ such that $(u, v)$ and $P_{1}$ form a $\delta$-fat path, i.e.,

$$
u_{g, \mu}(u, v) \gamma_{\mu}(u, v) \prod_{e \in P_{1}} \gamma_{\mu}(e) \geq \delta
$$


Let $P_{2}$ be the $v$ - $t$ path in the current highest-gain $\delta$-fat subtree. Since $P_{1}$ is some other $\delta$-fat path from $v$ to $t$, we have

$$
\operatorname{Gain}(v)=\prod_{e \in P_{2}} \gamma_{\mu}(e) \geq \prod_{e \in P_{1}} \gamma_{\mu}(e)
$$

Combining (7.1) and (7.2) we obtain $u_{g, \mu}(u, v) \gamma_{\mu}(u, v) \operatorname{Gain}(v) \geq \delta$, so we would not disregard arc $(u, v)$.

The following invariant ensures that at the beginning of the while loop, there are no $\delta$-fat arc with relabeled gain bigger than one. Thus, all arc costs are nonnegative and we are in a position to run the Dijkstra's variant.

Invariant 7.3.2. FATAUGmentATIONS maintains a lossy network $G_{g, \mu}^{\delta}$.

Proof. We prove by induction. The input network is assumed to be a lossy network. Before augmenting flow, the $\delta$-fat network is canonically relabeled so that $G_{g, \mu}$ is a lossy network and highest-gain $\delta$-fat paths have unit relabeled gain. Each $\delta$-fat augmentation is done along a highest-gain path, so only arcs with unit relabeled gain can be added to the residual graph.

We still need to rule out the possibility that a non $\delta$-fat arc with residual capacity becomes $\delta$-fat. Let $(u, v)$ be a non $\delta$-fat arc with residual capacity before the augmentation. Then, $(u, v)$ was disregarded, i.e.,

$$
u_{g, \mu}(u, v) \gamma_{\mu}(u, v) \operatorname{Gain}(v) \geq \delta
$$

Since augmentations are only along highest-gain $\delta$-fat paths, Gain $(v)$ does not increase. Hence, $(u, v)$ cannot become $\delta$-fat. 
Lemma 7.3.3. Procedure FatAugmentations $(G, \delta)$ outputs a flow $g$ of value at least $\mathrm{OPT}(G)-m \delta$.

Proof. Procedure FatAugmentations outputs a flow $g$ such that $G_{g}^{\delta}$ has no augmenting paths or flow-generating cycles. Let $g^{*}$ be an optimal flow in $G$. We can decompose the residual pseudoflow $g^{*}-g$ into at most $m$ elementary pseudoflows, as guaranteed by Theorem 2.3.3. All arcs in the decomposition are in $G_{g}$. The only elementary flows that generate excess at the sink are augmenting paths and GAPs. Therefore, it suffices to show that each of these elementary pseudoflows contributes at most $\delta$ to the maximum excess that can be sent to the sink.

First we consider augmenting paths. By construction there are no $\delta$-fat augmenting paths in $G_{g}$. Since each arc in the decomposition is in $G_{g}$, each augmenting path can only bring less than $\delta$ units of excess to the sink.

Now we consider GAPs. Since $G_{g}^{\delta}$ is a lossy network, there are no flow-generating cycles induced by $\delta$-fat arcs in $G_{g}$. Since every arc in the decomposition is also in $G_{g}$, every GAP in the decomposition has at least one arc in the flow-generating cycle, say $(v, w)$, that is not $\delta$-fat. The amount of flow that the GAP can generate at the sink is bounded above by the fatness of the $(v, t)$ path in the GAP, which is less than $\delta$. Thus, each GAP can only bring less than $\delta$ units of excess to the sink.

The next lemma bounds the number of fat-paths that need to be computed.

Lemma 7.3.4. During Procedure FatAugmentations $(G, \delta)$, there are at most $n+\mathrm{OPT}(G) / \delta$ augmentations. 
Proof. Each augmentation either uses up all of the excess at a node or increases the excess at the sink by at least $\delta$.

The procedure FatAugmentations can be implemented much like Dijkstra's shortest path algorithm. Using the Fredman-Tarjan [17] implementation with Fibonacci heaps, we obtain the following lemma.

Lemma 7.3.5. Procedure FATAugmentations computes each highest-gain $\delta$-fat tree in $\mathcal{O}(m+n \log n)$ time.

\subsection{Radzik's Fat-Path Variant}

The bottleneck computation in the original Fat-Path algorithm is canceling flowgenerating cycles. Radzik's variant [49] reduces the bottleneck by canceling only residual flow-generating cycles with sufficiently big gains. The CANCELCyCLES procedure quickly cancels flow-generating cycles with big gains, but requires significantly more time to cancel the remaining flow-generating cycles. For example, all flow-generating cycles with gains exceeding $1+1 / p(m)$, for any fixed polynomial $p(m)$, can be canceled in $\tilde{\mathcal{O}}(m n \log B)$, but $\tilde{\mathcal{O}}\left(m n^{2} \log B\right)$ time is required to cancel all flow-generating cycles. The remaining flow-generating cycles are obscured by appropriately decreasing the gain factors in the current computation. Since not all of the flow-generating cycles are actually canceled, analyzing the precision of the resulting solution is technically complicated. This idea leads to the following theorem of Radzik [49]: 
Theorem 7.4.1. In $\tilde{\mathcal{O}}\left(m^{2}+m n \log \log B\right) \log (1 / \xi)$ time Radzik's Fat-Path variant computes a $\xi$-optimal flow; in $\tilde{\mathcal{O}}\left(m^{3} \log B+m^{2} n \log B \log \log B\right)$ time it computes an optimal flow.

\subsection{Rounded Fat-Path}

In this section we present a new Fat-Path variant. Our variant runs the Fat-Path algorithm in the rounded network. This allows us to obtain an improved complexity bound on the CANCELCYCLES procedure, which is the bottleneck computation. By canceling all flow-generating cycles, we overcome the technical difficulties associated with Radzik's variant. Our rounding is done in a network with no residual flowgenerating cycles, which makes the quality of the resulting solution easy to analyze. Subsequent calls to the CANCELCYCLES procedure are performed in a pre-rounded network, which enables us to achieve an improved complexity bound.

\subsubsection{Rounded Fat-Path}

Algorithm Rounded Fat-Path (RFP) runs the original Fat-Path algorithm in a rounded network. The input is a lossy network $G$ and an error parameter $\xi$. First, RFP rounds down the gains to powers of $b=(1+\xi)^{1 / n}$, as described in Section 4.1. We note that if $G$ is a lossy network, then so is the rounded network, which we denote by $H$. Then Algorithm RFP runs the original Fat-Path algorithm on the rounded network. Algorithm RFP is given in Figure 7.5. 
Input: lossy network $G$, error parameter $0<\xi<1$

Output: $2 \xi$-optimal flow $g$

Set base $b=(1+\xi)^{1 / n}$ and round gains in network $G$ to powers of $b$

Let $H$ be resulting network

$h \leftarrow \operatorname{FAT}-\operatorname{Path}(H, \xi)$

Return $g \leftarrow$ interpretation of $h$ in $G$

Figure 7.5: Algorithm Rounded Fat-Path

Theorem 7.5.1. If $G$ is a lossy network then Algorithm RFP outputs a $2 \xi$-optimal flow in $\tilde{\mathcal{O}}\left(m^{2}+m n \log B \log (1 / \xi)\right) \log (1 / \xi)$ time.

Proof. Theorem 7.2.5 implies that the flow $h$ is $\xi$-optimal in network $H$. Theorem 4.1.1 says that the interpretation of the flow $h$ in the original network is a $2 \xi$-optimal flow.

As in the proof of Theorem 7.2.5, the bottleneck computations are procedures FatAugmentations and CancelCycles. As before, FatAugmentations requires $\tilde{\mathcal{O}}\left(m^{2}\right)$ per invocation. Since all of the gain factors are powers of $b$, Theorem 8.1.10 says that CANCELCYCLES requires $\tilde{\mathcal{O}}(m n \log C)$ time per invocation, where $C \leq n^{2} \xi^{-1} \log B$

\subsubsection{Recursive Rounded Fat-Path}

Algorithm RFP computes a $\xi$-optimal flow in $\tilde{\mathcal{O}}\left(m^{2}+m n \log \log B\right)$ time when $\xi^{-1}$ is $\mathcal{O}(p(m))$ for any polynomial $p(m)$, but does not compute optimal flows faster than the original Fat-Path algorithm. Our new recursive version computes nearly 
optimal and optimal flows faster than the original Fat-Path algorithm. The idea is that we can compute a $\xi$-optimal flow from two $\sqrt{\xi}$-optimal flows, as described in Section 4.1. In each recursive call, we re-round the network. The benefit is roughly to decrease the average value of $C$ from $\mathcal{O}\left(n^{2} \xi^{-1} \log B\right)$ to $\mathcal{O}\left(n^{2} \log B\right)$ because $G$ is a "pre-rounded" network.

The input to Algorithm Recursive Rounded Fat-Path (RRFP) is a network $G$, an upper bound $\Delta \geq \operatorname{OPT}(G)$ on the optimal value and an error parameter $\epsilon$. We assume the gain factors in the input network have already been pre-rounded to powers of $\left(1+\epsilon^{2}\right)^{1 / n}$; this will be the case when the algorithm is called recursively. The output is a flow of value at least $\operatorname{OPT}(G)-\epsilon \Delta$. With appropriate input parameters we can use RRFP to find a $\xi$-optimal flow in G. Algorithm RRFP first cancels all residual flow-generating cycles. If the error parameter is sufficiently course, FatAugmentations efficiently returns a flow of the desired precision. Otherwise, we round gain factors in the relabeled residual graph $G_{g, \mu}$ down to powers of $b=(1+\epsilon / 2)^{1 / n}$. Let $H$ denote the resulting rounded network. Now RRFP recursively calls itself with network $H$ and error parameter $\sqrt{\epsilon / 2}$. Let $h$ denote the resulting flow. Then RRFP recursively calls itself again in the resulting residual network $H_{h}$ with the same error parameter. Let $h^{\prime}$ denote the resulting flow. Let $g^{\prime}$ denote the interpretation of flow $h+h^{\prime}$ in $G_{g}$. RRFP outputs the flow $g+g^{\prime}$; we show below that it has the desired level of precision. Algorithm RRFP is described in Figure 7.6.

The following lemma shows that RRFP outputs a flow of the desired quality. 


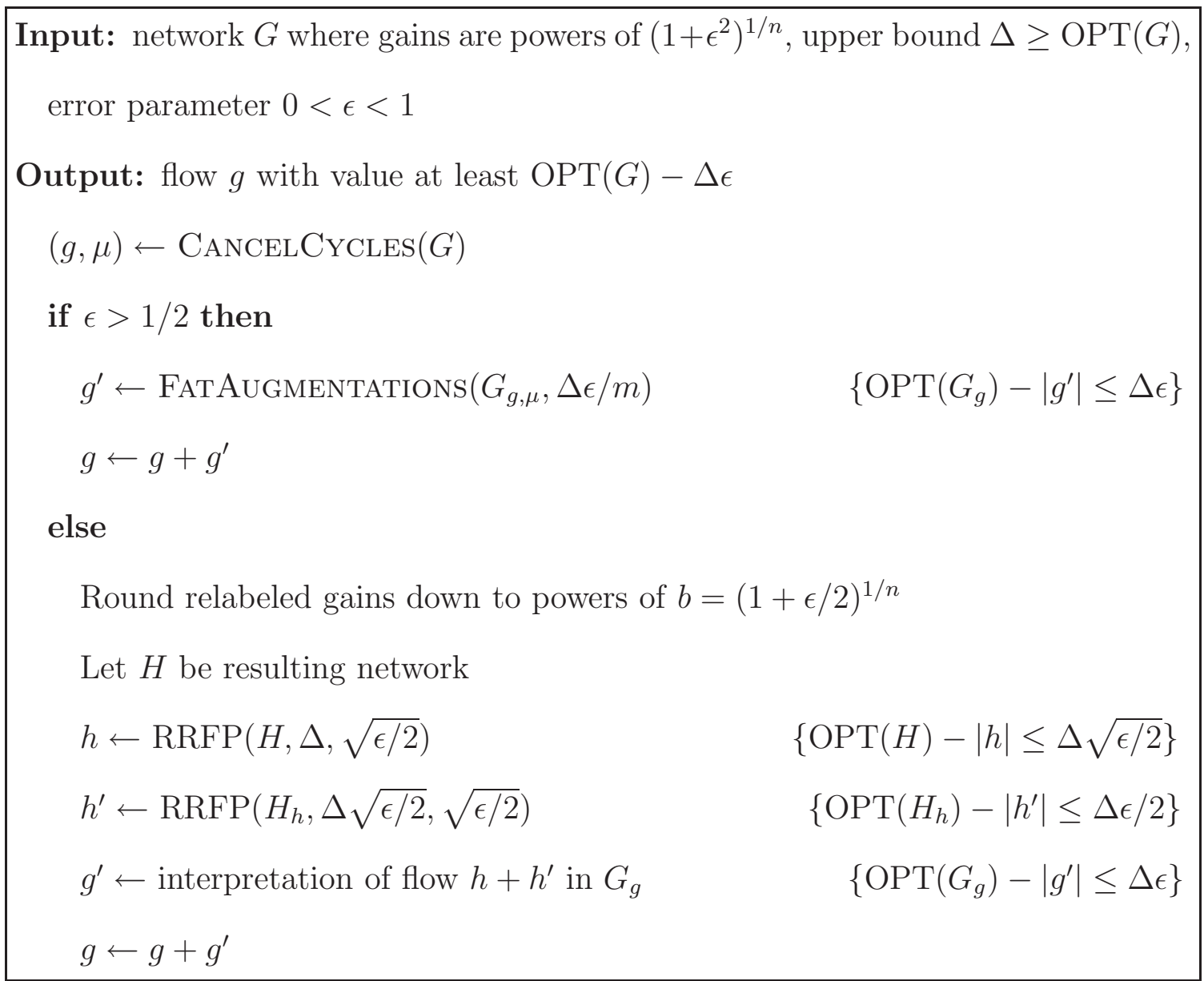

Figure 7.6: Algorithm Recursive Rounded Fat-Path

Lemma 7.5.2. If $\Delta \geq \operatorname{OPT}(G)$ and $0<\epsilon<1$ then $\operatorname{RRFP}(G, \Delta, \epsilon)$ returns a flow in network $G$ of value at least $\mathrm{OPT}(G)-\Delta \epsilon$.

Proof. The proof is by induction on the depth of the recursion tree. At depth zero, when $\epsilon>1 / 2$, by Lemma 7.3.3 FatAugmentations $\left(G_{g}, \mu, \Delta \epsilon / m\right)$ returns a flow $g^{\prime}$ of value $\left|g^{\prime}\right| \geq \operatorname{OPT}\left(G_{g}\right)-m(\Delta \epsilon / m)=\mathrm{OPT}\left(G_{g}\right)-\Delta \epsilon$. Hence

$$
\operatorname{OPT}(G)-\left|g+g^{\prime}\right|=\operatorname{OPT}\left(G_{g}\right)-\left|g^{\prime}\right| \leq \Delta \epsilon
$$


Otherwise when $\epsilon \leq 1 / 2$

$$
\begin{aligned}
\mathrm{OPT}(G)-\left|g+g^{\prime}\right| & \leq \mathrm{OPT}(G)-|g|-|h|-\left|h^{\prime}\right| \\
& \leq(1-\epsilon / 2) \operatorname{OPT}(G)+\Delta \epsilon / 2-|g|-|h|-\left|h^{\prime}\right| \\
& \leq(1-\epsilon / 2) \operatorname{OPT}\left(G_{g}\right)-|h|-\left|h^{\prime}\right|+\Delta \epsilon / 2 \\
& \leq \operatorname{OPT}(H)-|h|-\left|h^{\prime}\right|+\Delta \epsilon / 2 \\
& =\operatorname{OPT}\left(H_{h}\right)-\left|h^{\prime}\right|+\Delta \epsilon / 2 \\
& \leq \Delta \epsilon / 2+\Delta \epsilon / 2=\Delta \epsilon .
\end{aligned}
$$

The first inequality follows since flow interpretation can only create additional excesses. The second inequality holds since $\mathrm{OPT}(G) \leq \Delta$ by Invariant 7.2.2. Since $H$ is the rounded network, the fourth inequality holds by Theorem 4.1.1. The final inequality follows by induction on the second recursive call.

We need to check that the inductive hypothesis for the second recursive call is valid, i.e., $\Delta \sqrt{\epsilon / 2} \geq \operatorname{OPT}\left(H_{h}\right)$. This follows by induction on the first recursive call, since it outputs a flow $h$ that satisfies $\operatorname{OPT}\left(H_{h}\right)=\mathrm{OPT}(H)-|h| \leq \Delta \sqrt{\epsilon / 2}$. Also, we need to check that the inductive hypothesis for the first recursive call is valid. It is satisfied since $\Delta \geq \mathrm{OPT}(G) \geq \mathrm{OPT}(H)$.

Now, we can bound the running time of algorithm RRFP.

Lemma 7.5.3. If $\epsilon^{-1}=B^{\mathcal{O}(m)}$ then in $\tilde{\mathcal{O}}\left(m^{2}+m n \log \log B\right) \log (1 / \epsilon)$ time Algorithm $\operatorname{RRFP}(G, \Delta, \epsilon)$ terminates.

Proof. The bottleneck computations are procedures CANCELCyCLES and FATAugMEntations. There are $\log \log \epsilon^{-1}$ levels in the recursion tree. RRFP calls FA- 
TAugmentations only at depth zero in the recursion tree. Thus, there $\operatorname{are} \log (1 / \epsilon)$ calls to FatAugmentations; Lemma 7.2.1 implies that each call requires $\tilde{\mathcal{O}}\left(m^{2}\right)$ time.

Now, we bound the time due to calls to CanCelCycles. During an invocation of RRFP with error parameter $\epsilon$, RRFP calls CANCELCYCLES once and recursively calls RRFP twice with error parameter $\sqrt{\epsilon / 2}$. Since all of the gains are powers of $\left(1+\epsilon^{2}\right)^{1 / n}$, the call to CANCELCYCLES requires $\mathcal{O}(m n \log n \log (n C))$ where $C=$ $n^{2} \epsilon^{-2} \log B$. Let $T(m, n, \epsilon)$ be an upper bound on the time required to execute $\operatorname{RRFP}(G, \epsilon, \Delta)$. Then $T$ solves the recurrence:

$$
T(m, n, \epsilon)=\mathcal{O}\left(m n \log n \log \left(\frac{n^{3} \log B}{\epsilon^{2}}\right)\right)+2 T(m, n, \sqrt{\epsilon / 2}) .
$$

Radzik [49] showed that

$$
T(m, n, \epsilon)=\mathcal{O}\left(m n \log n \log (n \log B) \log \epsilon^{-1}\right)+\mathcal{O}\left(m n \log n \log \epsilon^{-1} \log \log \epsilon^{-1}\right)
$$

solves the recurrence (7.3). When $\epsilon^{-1}$ is $B^{\mathcal{O}(m)}$, which will be the case even to compute optimal flows, this simplifies to: $T(m, n, \epsilon)=\mathcal{O}\left(m n \log n \log (m \log B) \log \epsilon^{-1}\right)$.

Theorem 7.5.4. In $\tilde{\mathcal{O}}\left(m^{2}+m n \log \log B\right) \log (1 / \xi)$ time Algorithm RRFP computes a $\xi$-optimal flow. An extra $\tilde{\mathcal{O}}\left(m n^{2} \log B\right)$ preprocessing time is required if $G$ has residual flow-generating cycles. Algorithm RRFP computes an optimal flow in $\mathcal{O}\left(m^{3} \log B+m^{2} n \log B \log \log B\right)$ time.

Proof. To compute a $\xi$-optimal flow, we initialize $\Delta$ with a value $\Delta_{0}$ that satisfies $\operatorname{OPT}(G) \leq \Delta_{0} \leq m \operatorname{OPT}(G)$. This can be done efficiently as described in Corol- 
lary 7.1.4. By Lemma 7.5.2, the flow $g$ that $\operatorname{Algorithm} \operatorname{RRFP}\left(G, \Delta_{0}, \xi / n\right)$ returns satisfies:

$$
|g| \geq \operatorname{OPT}(G)-(\xi / n) \Delta_{0} \geq(1-\xi) \operatorname{OPT}(G) .
$$

The complexity bound for computing approximate flows follows from Lemma 7.5.3. To compute an optimal flow, it suffices by Lemma 2.5.2 to compute a $B^{-3 m}$-optimal flow.

Complexity Bounds. The original Fat-Path variant of Goldberg, Plotkin, and Tardos [20] solves the generalized maximum flow problem in $\tilde{\mathcal{O}}\left(m^{2} n^{2} \log ^{2} B\right)$ time. It computes $\xi$-optimal flows in $\tilde{\mathcal{O}}\left(m n^{2} \log B\right) \log (1 / \xi)$ time. Radzik [48] provided a strongly-polynomial complexity of $\tilde{\mathcal{O}}\left(m^{2} n\right) \log (1 / \xi)$ time for computing $\xi$-optimal flows. Radzik's [49] Fat-Path variant computes optimal flows in $\tilde{\mathcal{O}}\left(m^{3} \log B+\right.$ $\left.m^{2} n \log B \log \log B\right)$ time and $\xi$-optimal flows in $\tilde{\mathcal{O}}\left(m^{2}+m n \log \log B\right) \log (1 / \xi)$ time. Our Fat-Path variant matches these best known worst-case complexity bounds. 


\section{Chapter 8}

\section{Canceling Flow-Generating Cycles}

In this chapter we describe a method for converting one generalized flow into another generalized flow whose residual graph contains no flow-generating cycles. In the process additional excesses, but no deficits, may be created. By eliminating flowgenerating cycles, we can subsequently perform many generalized flow computations more efficiently. The method was first used by Goldberg, Plotkin, and Tardos [20] in their Fat-Path algorithm.

Our procedure is a variant of the CANCELCyCLES procedure of [20]. With our variant, we ensure an additional property: the node potentials change by only relatively small amounts. This new property is useful for improving the complexity of some generalized flow algorithms, including the primal-dual algorithm described in Chapter 5. Also, Radzik [48] showed that with slight modifications, CANCELCyCLES runs in strongly polynomial-time; our variant allows a simpler proof of this fact. 


\subsection{Cancel Cycles}

In this section, we review the CANCELCYCLES procedure of Goldberg, Plotkin, and Tardos [20]. The basic approach is to repeatedly cancel residual flow-generating cycles, i.e., send flow along a cycle, until one (or more) residual arcs become saturated. By canceling flow-generating cycles, we may create additional node excesses, but no deficits. This is the generalized flow analog of Klein's [40] minimum cost flow cycle-canceling algorithm with $\operatorname{costs} c=-\log \gamma$. Using this cost function, negative cost cycles correspond to flow-generating cycles.

Canceling arbitrary negative cost cycles may result in an exponential-time algorithm. Goldberg and Tarjan [23] obtained a polynomial-time minimum cost flow algorithm by repeatedly canceling the residual cycle with the minimum mean cost; recall the mean cost of a cycle $\Gamma$ is $\mu(\Gamma)=c(\Gamma) /|\Gamma|$, where $c(\Gamma)=\sum_{e \in \Gamma} c(e)$. Goldberg and Tarjan [23] also designed a more efficient cycle-canceling algorithm called Cancel-And-Tighten; the crucial idea is to maintain node potentials and only approximate the strategy of canceling minimum mean cycles.

The CancelCycles procedure of Goldberg, Plotkin, and Tardos [20] adapts the CANCEL-And-Tighten algorithm to generalized flows, using the cost function $c=-\log _{b} \gamma$, for some base $b>0$. CAnCELCyCles is divided into cost-scaling phases. In each $\epsilon$-phase, the procedure maintains a generalized flow $g$ and node potentials $\pi$ that satisfy the $\epsilon$-complementary slackness conditions:

$$
\forall(v, w) \in G_{g}: \quad c_{\pi}(v, w)=c(v, w)-\pi(v)+\pi(w) \geq-\epsilon .
$$


The node potentials are analogous to the node labels described in Section 2.3.2; the node label corresponding to $\pi(v)$ is $\mu(v)=b^{\pi(v)-\pi(t)}$, so that $\mu(t)$ is normalized to one.

Remark 8.1.1. If $g$ and $\pi$ are 0 -complementary slack then $G_{g, \mu}$ is a lossy network because

$$
\forall(v, w) \in G_{g}: \quad \gamma_{\mu}(v, w)=\gamma(v, w) \mu(v) / \mu(w)=\gamma(v, w) b^{\pi(v)-\pi(w)} \leq 1
$$

where the last inequality follows from the definition of 0-complementary slackness.

There is a very close connection between the $\epsilon$-complementary slackness conditions and minimum mean cost cycles. Given a generalized flow $g$, we define $\epsilon(g)$ to be the minimum value of $\epsilon$ for which $g$ is $\epsilon$-complementary slack for some node potentials $\pi$. That is

$$
\epsilon(g)=\min _{\pi}\{\epsilon: g, \pi \text { satisfies }(8.1)\}
$$

With respect to $g$, we denote the value of the minimum mean cost residual cycle by

$$
\mu(g)=\min \left\{\mu(\Gamma): \Gamma \in G_{g}\right\}
$$

The strong duality between $\epsilon$-complementary slackness and minimum mean cost cycles is captured by the following lemma.

Lemma 8.1.2. Let $g$ be a non-optimal flow. Then $\epsilon(g)=-\mu(g)$.

Proof. The lemma follows immediately from linear programming strong duality. We give a more direct proof. 
Suppose $g$ and $\pi$ are $\epsilon$-complementary slack. By definition, the reduced cost of every residual arc is at least $-\epsilon$, and hence the mean reduced cost of any cycle is at least $-\epsilon$. Thus, $\mu(g) \geq-\epsilon(g)$, since the mean cost of a cycle is equal to the mean reduced cost of a cycle.

Now, we show $\mu(g) \leq-\epsilon(g)$. Let $\Gamma$ be a residual cycle in $G_{g}$ that has minimum mean cost value $\mu(g)$ Suppose that we replace each arc cost $c(v, w)$ by $\bar{c}(v, w)=$ $c(v, w)-\mu(g)$. This increases the mean costs of every residual cycle by $-\mu(g)$. Hence, $\Gamma$ now has zero (reduced) cost and there are no negative (reduced) cost residual cycles in $G_{g}$, with respect to the updated costs. Let $\pi(v)$ denote the cheapest costs from node $v$ to the sink. using costs $\bar{c}$. The shortest path optimality conditions imply

$$
\forall(v, w) \in E_{g}: \quad \bar{c}_{\pi}(v, w)=\bar{c}(v, w)-\pi(v)+\pi(w) \geq 0
$$

In other words, $g$ and $\pi$ are $-\mu(g)$-complementary slack. Thus $\epsilon(g) \geq-\mu(g)$.

Remark 8.1.3. Lemma 8.1.2 implies that it is possible to compute $\epsilon(g)$ by computing the minimum mean cost cycle value. This can be done in $\mathcal{O}(m n)$ time. See Section 2.2.2 for more details.

During a phase, CANCELCyCles maintains a flow $g$ and node potentials $\pi$. Instead of repeatedly canceling minimum mean cost cycles, it repeatedly cancels totally negative cycles, until no such cycles remain. A totally negative cycle is a residual cycle for which every arc has negative reduced cost. After an $\epsilon$-phase, new node potentials are computed, and the error parameter $\epsilon$ is decreased by a factor of $(1-1 / n)$. Initially we choose $\epsilon=C=\max _{e \in E} c(e)$, and when $\epsilon$ is sufficiently 
small, we show that there are no residual flow-generating cycles. Procedure CANCELCYCLES is described in Figure 8.1.

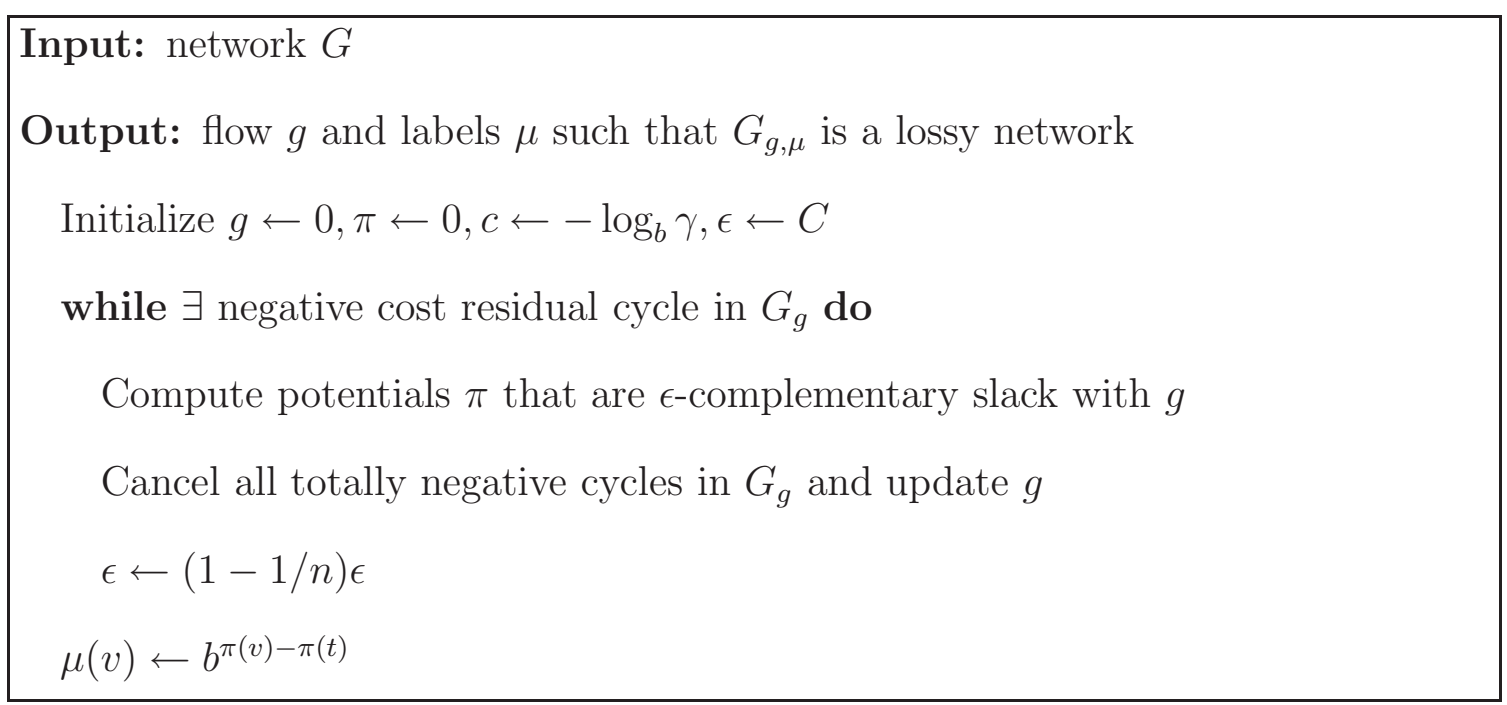

Figure 8.1: Subroutine Cancel Cycles

Invariant 8.1.4. In the first step of a phase in CANCELCYCLES there exist potentials $\pi$ that are $\epsilon$-complementary slack with the current flow. Also, $\epsilon$-complementary slackness is preserved while canceling totally negative cycles.

Proof. We prove both claims together by induction. The lemma is true at the beginning of the first phase since $g=0$ and $\pi=0$ are $\epsilon$-complementary slack for $\epsilon=C$.

First, we show that canceling a totally negative cycle maintains $\epsilon$-complementary slackness. Canceling a totally negative cycle may only add the reversals of these cycle arcs into the residual network, and each of these reverse arcs has positive reduced cost. 
Now, we demonstrate the existence of potentials which are $\epsilon$-complementary slack with the current flow at the beginning of a phase. At the end of the previous phase, $\epsilon$ was decreased by a factor of $(1-1 / n)$. At this point, by induction, all residual arcs have reduced cost at least $-\epsilon$. Also, since there are no totally negative cycles, at least one arc in each residual cycle has nonnegative reduced cost. This implies $\mu(g) \geq-\epsilon(1-1 / n)$. Lemma 8.1 .2 asserts that there exists node potentials that are $\epsilon(g)$-complementary slack with the current flow $g$.

The next lemma says that at the beginning of a phase, we can compute the node potentials efficiently.

Lemma 8.1.5. At the beginning of an $\epsilon$-phase, we can find node potentials $\pi$ that are $\epsilon$-complementary slack with the current flow $g$ in $\mathcal{O}(m)$ time.

Proof. After canceling totally negative cycles in the previous phase, the subgraph induced by negative reduced cost residual arcs is acyclic and induces a topological ordering of the nodes $o: V \rightarrow\{0, \ldots, n-1\}$ such that if $(v, w) \in G_{g}$ and $c_{\pi}(v, w)<$ 0 then $(v, w)$ is a forward arc in the ordering, i.e., $o(v)<o(w)$. We compute the topological ordering $o$ and increase the node potential $\pi(v)$ by $o(v) \epsilon / n$. This increases the reduced cost of forward arcs by at least $\epsilon / n$, and decreases the reduced cost of backwards arcs by at most $(n-1) / n$. All negative reduced cost residual arcs are forward arcs in the ordering; now, these arcs have reduced cost at least $-(1-1 / n) \epsilon$. All backwards arcs previously had nonnegative reduced cost; now these arcs have reduced cost at least $-(1-1 / n) \epsilon$. 
The next two lemmas say that if $\epsilon$ is sufficiently small, then the current flow has no residual flow-generating cycles. This can then be used to bound the number of phases and serve as a stopping condition. As an alternate stopping condition. CANCELCyCles directly checks for flow-generating (negative-cost) cycles. This condition can be checked in $\mathcal{O}(m n)$ time with a shortest path computation, e.g., see Section 2.2.1. However, checking this condition may dominate the overall complexity, so we only check for negative cost cycles every $n$ iterations.

Lemma 8.1.6. Suppose that the costs $c=-\log _{b} \gamma$ are integral for some $b>0$. Let $g$ and $\pi$ be $\epsilon$-complementary slack for $\epsilon<1 / n$. Then $G_{g}$ has no flow-generating cycles.

Proof. Let $\Gamma$ be a cycle in $G_{g}$. Then $c(\Gamma)=c_{\pi}(\Gamma)=\sum_{e \in \Gamma} c_{\pi}(e) \geq-n \epsilon>-1$. By assumption, the costs are integral. Hence $c(\Gamma) \geq 0$, or equivalently, $\Gamma$ is not a flow-generating cycle.

Lemma 8.1.7. Suppose the gains are ratios of integers between 1 and B. Let $g$ and $\pi$ be $\epsilon$-complementary slack for $\epsilon<1 /\left(2 n B^{n} \log b\right)$. Then $G_{g}$ has no flow-generating cycles.

Proof. Let $T$ denote the maximum product of gain numerators on a simple cycle, and note that $T \leq B^{n}$. Let $\Gamma$ be a cycle in $G_{g}$ with $\gamma(\Gamma)>1$. Then $\gamma(\Gamma) \geq 1+T^{-1}$. This implies that its mean cost

$$
\mu(\Gamma) \leq \frac{-\log _{b}\left(1+T^{-1}\right)}{n}=\frac{-\log _{2}\left(1+T^{-1}\right)}{n \log b} \leq \frac{-1}{2 n T \log b}
$$


where the last inequality follows by taking a Taylor expansion of $\log _{2}(1+x)$ for $x \leq 1$. Now, by Lemma 8.1.2,

$$
\epsilon \geq \epsilon(g)=-\mu(g) \geq-\mu(\Gamma) \geq \frac{1}{2 n T \log b} \geq \frac{1}{2 n B^{n} \log b}
$$

Lemma 8.1.8. If the gains are given as ratios of integers between 1 and $B$ then CAnCElCyCles requires $\mathcal{O}\left(n^{2} \log B\right)$ phases. If the costs are integers no bigger than $C$, it requires $\mathcal{O}(n \log (n C))$ phases.

Proof. Each phase decrease $\epsilon$ by a factor of $(1-1 / n)$. Thus, after $\mathcal{O}(n)$ phases, $\epsilon$ decreases by at least a factor of two. Initially $\epsilon=C$. If the costs $c=-\log _{b} \gamma$ are integers no bigger than $C$, then Lemma 8.1.6 implies that we can stop when $\epsilon<1 / n$. If the gains are ratios of integers between 1 and $B$, then Lemma 8.1.7 implies that we can stop when $\epsilon<1 /\left(2 n B^{n} \log b\right)$. Note that $C \leq \log _{b} B$.

Now, we give a complexity bound for canceling totally negative cycles. Each time we cancel a totally negative cycle, we saturate at least one arc with negative reduced cost, and we do not create any new such arcs. Thus, we cancel at most $m$ cycles per phase. Finding and canceling a single totally negative cycle, while creating only excesses and no deficits, can easily be done in $\mathcal{O}(m)$ time. This leads to a $\mathcal{O}\left(m^{2}\right)$ time method. By marking nodes that do not participate in totally negative cycles, we can improve the complexity to $\mathcal{O}(m n)$ time.

Using the dynamic tree data structure of Sleator and Tarjan [51], we can improve the complexity bound further. One of the primitive dynamic tree operations is 
pushing the same amount of flow along every arc in a tree path. In non-generalized networks, we can also use the dynamic tree data structure to push flow around a cycle that consist of a tree path plus one extra arc. CancelCycles pushes flow around totally negative cycles; this means that each arc in the cycle has a relabeled gain factor exceeding one, and we would want to push increasing amounts of flow along each successive arc in the cycle. Thus, the dynamic tree data structure does not apply directly. However, we can directly use the dynamic tree data structure by abstracting the notion of canceling a cycle; we now allow excesses to be created at several nodes when canceling a cycle. We note that this does not affect any of our previous analysis. Since all arcs have relabeled gain above one, by pushing the same amount of flow along every arc in the cycle, we create excesses at every cycle node. This idea leads to the following lemma from [20].

Lemma 8.1.9. Canceling all totally negative cycles requires $\mathcal{O}(m \log n)$ time.

Theorem 8.1.10. If the gains are given as ratios of integers between 1 and $B$ then CANCELCyCles requires $\tilde{\mathcal{O}}\left(m n^{2} \log B\right)$ time. If the costs are integers no bigger than $C$, it requires $\tilde{\mathcal{O}}(m n \log C)$ time.

Proof. The bottleneck computation is canceling all totally negative cycles. By Lemma 8.1.9, each phase requires $\tilde{\mathcal{O}}(m)$ time. The number of phases is given by Lemma 8.1.8. 


\subsection{Our Cancel Cycles Variant}

In this section, we describe a new variant of CANCELCYCLES. Our variant ensures that the node potentials change by only a relatively small amount while canceling cycles. Our procedure uses two types of potential updating. With respect to a flow $g$, loose updating is similar to the potential updating in the original CANCELCYCLES procedure. It requires $\mathcal{O}(m)$ time, but does not guarantee to decrease $\epsilon$ by the maximum amount. If there are no totally negative cycles, it decreases $\epsilon$ by at least a factor of $(1-1 / n)$. We also use tight updating, which updates $\epsilon=\epsilon(g)$ and computes potentials that are $\epsilon(g)$-complementary slack with $g$. It reduces $\epsilon$ by the maximum possible amount, but is relatively expensive. It involves computing a minimum mean cost cycle and requires $\mathcal{O}(m n)$ time. We also introduce a third type of potential updating which we call medium updating. It offers a middleground between loose and tight updating. It is much cheaper than tight updating, yet more effective than loose updating. The three types of potential updating are described in more detail below.

The input to our CAnCElCyCles variant, which we call CAnCElCyCles2, is a lossy network $G$. It is divided into cost-scaling phases. In each phase, the procedure balances the amount of work between canceling totally negative cycles, loose updating, and tight updating. As with CANCELCyCLES procedure, in each $\epsilon$-phase, our procedure maintains a flow $g$ and potential $\pi$ that are $\epsilon$-complementary slack. The procedure begins a cost-scaling phase by performing tight updating. Next, the algorithm cancels a minimum mean cost cycle. Then it alternates between the 
following two operations: first it cancels all totally negative cycles, then it performs loose updating. The procedure CANCELCyCLES2 is described in Figure 8.2.

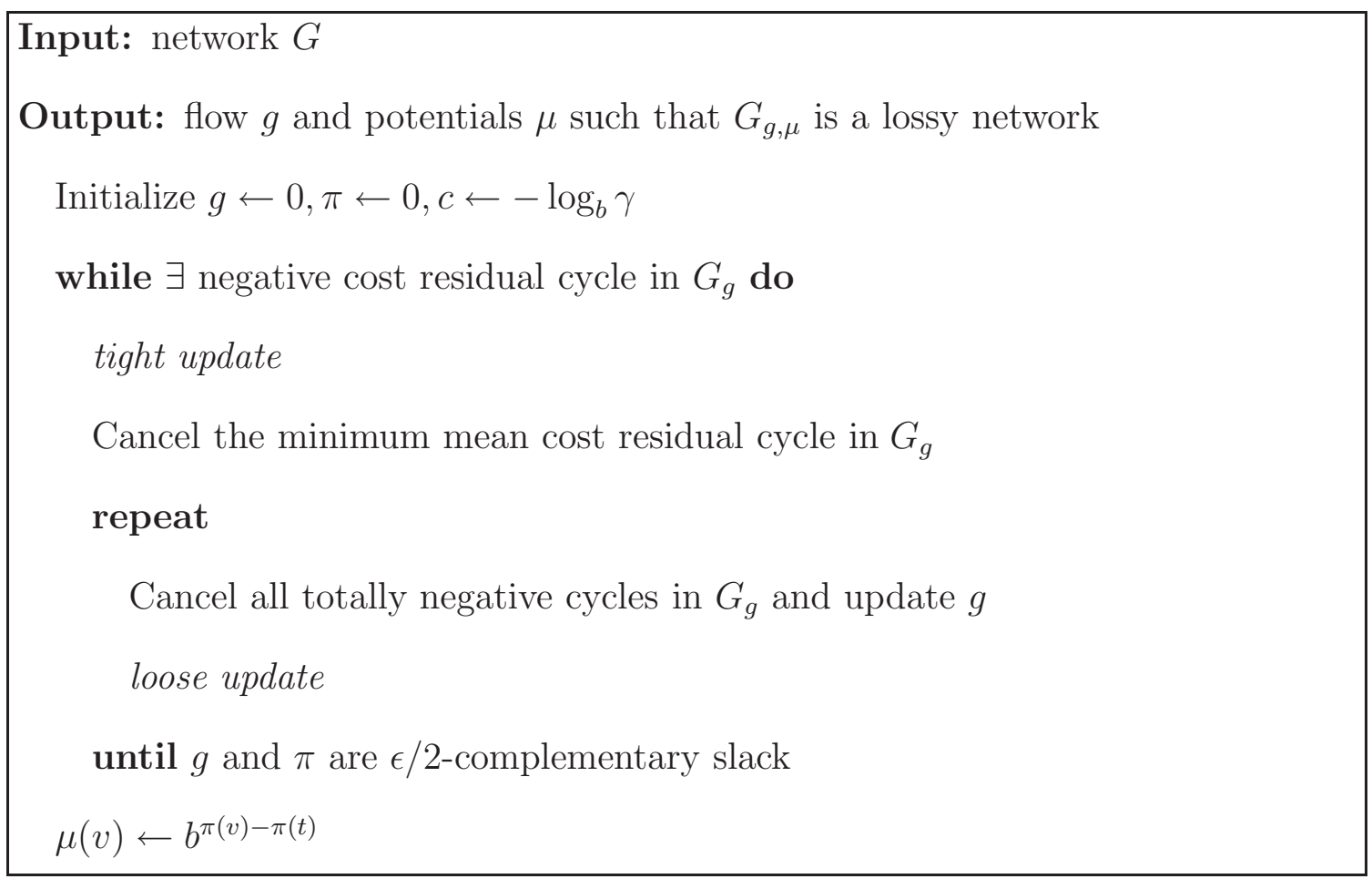

Figure 8.2: Subroutine Cancel Cycles2

Tight Updating. Tight updating sets $\epsilon$ to $\epsilon(g)$ and finds new potentials $\pi$ that are $\epsilon(g)$-complementary slack with the current flow $g$. This can be done with a single minimum mean cost cycle computation [1]: there is a natural set of node potentials associated with this computation, and they are $\epsilon(g)$-complementary slack with the flow $g$. However, these potentials are not good enough for our purposes; they may be too large. For our analysis, we compute $\epsilon(g)$ and use this value to find new potentials which are relatively small. 
Lemma 8.2.1. Suppose $g$ and $\pi$ are $\epsilon$-complementary slack. Then, there exists node potentials $p$ such that $g$ and $\pi+p$ are $\epsilon(g)$-complementary slack and $0 \leq p \leq$ nє. Moreover, given $\epsilon(g)$, we can find such potentials $p$ with a single shortest path computation.

Proof. First, we compute $\epsilon(g)=-\mu(g)$. Then, we define $\bar{c}: E_{g} \rightarrow \Re$ by $\bar{c}(v, w)=$ $c_{\pi}(v, w)+\epsilon(g)$, so that there are no negative cost cycles in $G_{g}$ with respect to the new cost function $\bar{c}$. Let $q(v)$ denote the value of the cheapest (possibly trivial) residual path from $v$ to any other node using costs $\bar{c}$. Note that $q(v) \leq 0$ for every node $v$.

Now, we describe how to compute $q$ efficiently. Consider the network $G_{g}$ with $\operatorname{costs} \bar{c}$. We add a new artificial sink $t^{\prime}$ and zero cost arcs from every node to $t^{\prime}$. Since there are no negative cost cycles, we can compute $q$ using a single shortest path (from every node to $t^{\prime}$ ) computation. The shortest path optimality conditions imply:

$$
\forall(v, w) \in E_{g}: \quad q(v) \leq q(w)+\bar{c}(v, w)
$$

and

$$
\forall v \in V: \quad 0 \geq q(v) \geq n \min _{e \in E_{g}} \bar{c}(e) \geq n(\epsilon(g)-\epsilon) \geq-n \epsilon
$$

Consequently,

$$
\begin{aligned}
\forall(v, w) \in E_{g}: \quad c_{\pi+q}(v, w) & =c_{\pi}(v, w)-q(v)+q(w) \\
& =\bar{c}(v, w)-\epsilon(g)-q(v)+q(w) \\
& \geq-\epsilon(g) .
\end{aligned}
$$


The lemma then follows by choosing $p=q+n \epsilon$.

Additionally, it is easy to verify that after tight updating, each arc participating in a minimum mean cost residual cycle has reduced cost equal to $-\epsilon(g)$, implying that it is a totally negative cycle.

Loose Updating. Loose updating occurs when there are no more totally negative cycles. By increasing the potentials of a subset of nodes, we may create new arcs with negative reduced cost, and hence new totally negative cycles. CANCELCyClEs2 performs loose updating immediately after cancelling all totally negative cycles. At this point, there are no totally negative cycles; this induces a topological ordering of the nodes. We say $i \preceq j$ if there is an $i$-j residual path using only negative reduced arcs. Let $(v, w)$ be some residual arc with reduced cost below $-\epsilon / 2$; the phase terminates if no such arc exists. Loose updating increases by $\epsilon / 2$ the potential of each node $x \succeq w$.

The following invariant says that the procedure maintains a flow and node potentials that are $\epsilon$-complementary slack.

Invariant 8.2.2. During an $\epsilon$-phase, the algorithm maintains a flow $g$ and potentials $\pi$ that are $\epsilon$-complementary slack.

Proof. At the beginning of a phase, $\pi$ is chosen to be $\epsilon$-complementary slack with $g$, where $\epsilon=\epsilon(g)$. We prove by induction that within an $\epsilon$-phase, the procedure maintains this property. As in Invariant 8.1.4, $\epsilon$-complementary slackness is preserved when canceling totally negative cycles. 
Now, we show that $\epsilon$-complementary slackness is preserved during loose updating. Loose updating occurs only when there are no totally negative cycles. It increases the node potentials for some subset $S$ of nodes which have no negative reduced cost leaving arcs. This decreases the reduced cost of $\operatorname{arcs}$ leaving $S$ and increases the reduced cost of arcs entering $S$ by $\epsilon / 2$. But all arcs leaving $S$ previously had nonnegative reduced cost, so after the loose update they have reduced cost at least $-\epsilon / 2$.

Lemma 8.2.3. There are at most $n$ loose updates per phase.

Proof. By Invariant 8.2.2, during an $\epsilon$-phase the reduced cost of every residual arc is at least $-\epsilon$. Before a loose update, CANCELCyCles 2 selects an arc $(v, w)$ with reduced cost less than $-\epsilon / 2$. After the potential update, all arcs entering $w$ have reduced cost at least $-\epsilon / 2$. Thus, there can be at most $n$ loose updates per $\epsilon$-phase, as no new residual arcs with reduced cost less than $-\epsilon / 2$ are created.

Medium updating. We introduce a medium updating which is a middleground between loose and tight updating. After cancelling all totally negative cycles, we would ideally like to perform tight updating and compute $\epsilon(g)$ exactly. This is computationally expensive, so instead our procedure uses loose updating. Loose updating can be done in linear time, but it may not provide high quality node potentials. We propose a practical alternative.

Medium updating finds a value $\epsilon^{\prime}$ that is close to $\epsilon(g)$, without spending the time to find the actual minimum mean cost cycle. We only need to estimate $\epsilon(g)$ in networks that have no totally negative cycles. We exploit this fact. To do 
this efficiently, we imagine that, in addition to the original arcs, a zero cost link exists between every pair of nodes. We can efficiently find a minimum mean cost cycle in this modified network by computing a minimum mean cost path in the acyclic network induced by only negative cost arcs, without explicitly considering the imaginary zero cost arcs. Let $\epsilon^{\prime}$ denote the value of the minimum mean cost cycle in the modified network. Clearly $\epsilon^{\prime} \leq \epsilon(g)$, and it is not hard to see that $\epsilon^{\prime} \geq(1-1 / n) \epsilon$. We can binary search for $\epsilon^{\prime}$ using a shortest path computation in acyclic graphs. This requires only $\mathcal{O}(m)$ time per iteration. If we were to determine $\epsilon^{\prime}$ exactly, in $\tilde{\mathcal{O}}(n \log B)$ iterations the search interval would be sufficiently small. If the gains in the network are rounded to powers of $b=(1+\xi)^{1 / n}$ then $\tilde{\mathcal{O}}(\log C)$ iterations suffice, where $C=\mathcal{O}\left(n \xi^{-1} \log B\right)$. Since we are only estimating $\epsilon(g)$, there is no need to compute $\epsilon^{\prime}$ exactly either.

The following lemma gives a weakly-polynomial bound on the number of phases.

Lemma 8.2.4. If the gains are given as ratios of integers between 1 and $B$ then CAnCELCyCles2 requires $\mathcal{O}(n \log B)$ phases. If the costs are integers no bigger than $C$, it requires $\log (n C)$ phases.

Proof. Now, each phase decrease $\epsilon$ by a factor of at least two. The rest of the proof is the same as in Lemma 8.1.8.

The next lemma gives a strongly-polynomial bound on the number of phases. Radzik [48] showed that $\mathcal{O}(m \log n)$ phases suffice. Radzik introduces a new auxiliary parameter $\delta$; the value of $\delta$ at the beginning of a phase is defined as the minimum cost of a cycle canceled from the beginning of the phase until the end 
of the procedure. His analysis relies on showing that $\delta$ converges geometrically to zero. Our new algorithm allows a simpler proof the strongly-polynomial complexity bound. We show that after $\mathcal{O}(\log n)$ phases a new arc becomes fixed, i.e., the value of flow on this arc will not change during the rest of the procedure.

Lemma 8.2.5. CANCELCyCles2 requires $\mathcal{O}(m \log n)$ phases.

Proof. Within an $\epsilon$-phase, Lemma 8.2.3 says that the potential of a node increases by at most $n \epsilon / 2$ due to loose updating. Hence from the start of the $\epsilon$-phase until the termination of the procedure, a node's potential increases by at most $n \epsilon$ as a result of loose relabeling, since $\epsilon$ halves in each phase.

Following an $\epsilon$-phase, tight updating might increase node potentials in subsequent scaling-phases. Lemma 8.2.1 implies that in the next scaling-phase, a node potential increases by at most $n \epsilon / 2$. Since $\epsilon$ halves in each phase, this implies a node's potential increases by a total of at most $n \epsilon$ during all subsequent tight updatings.

Hence after the initial tight updating in an $\epsilon$-phase, a node's potential increases by at most $2 n \epsilon$, until the algorithm terminates. Hence if an arc's reduced cost is smaller than $-3 n \epsilon$ (after the initial tight updating) in some $\epsilon$-phase, then that arc is fixed, as its reduced cost will remain below $-\epsilon$ throughout the procedure.

In each phase, the first cycle canceled is a minimum mean cost cycle, say $\Gamma$. Let $\epsilon$ be the scaling parameter when $\Gamma$ is cancelled and let $\epsilon^{\prime}$ be the scaling parameter $2+\log n$ phases later. Then

$$
\mu(\Gamma)=-\epsilon \leq-4 n \epsilon^{\prime} .
$$


Hence, throughout the $\epsilon^{\prime}$-phase at least one arc $(v, w) \in \Gamma$ has reduced cost as small as $-4 n \epsilon^{\prime}$. But we pushed flow on $(v, w)$ in the $\epsilon$-phase, so it wasn't fixed then. Thus the $\operatorname{arc}(v, w)$ becomes fixed within $\mathcal{O}(\log n)$ phases.

Theorem 8.2.6. If the gains are given as ratios of integers between 1 and $B$ then CancelCyCles2 requires $\tilde{\mathcal{O}}\left(m n^{2} \log B\right)$ time. If the costs are integers no bigger than $C$, it requires $\tilde{\mathcal{O}}(m n \log n)$ time. In any case CANCELCYCLES2 requires $\tilde{\mathcal{O}}\left(m^{2} n\right)$ time. Moreover, it outputs a flow $g$ and node labels $\bar{B}^{-3 n} \leq \mu \leq \bar{B}^{3 n}$ such that $G_{g, \mu}$ is a lossy network, where $\bar{B}$ is the biggest gain of a residual arc in the original network.

Proof. The number of phases is bounded by Lemma 8.2.4 and Lemma 8.2.5. By Lemma 8.2.3 we cancel all totally negative cycles at most $n$ times per phase. This is the bottleneck computation; by Lemma 8.1.9, it requires $\mathcal{O}(m n \log n)$ time per phase.

Now we provide a bound on how much the potentials can increase. As in the proof of Lemma 8.2.5, within an $\epsilon$-phase, a node potential can increase by at most $n \epsilon$ due to tight updating, and by at most $n \epsilon / 2$ due to loose updating. Since $\epsilon$ halves in each phase, this implies that a node potential increases by at most $3 n \epsilon$ throughout the entire procedure. Initially $\pi=0, g=0$, and $\epsilon=\epsilon(g) \leq \log _{b} \bar{B}$. Thus $0 \leq \pi \leq 3 n \log _{b} \bar{B}$. The theorem then follows, since the node label corresponding to $\pi(v)$ is $\mu(v)=b^{\pi(v)-\pi(t)}$. 


\section{Bibliography}

[1] R. K. Ahuja, T. L. Magnanti, and J. B. Orlin. Network Flows: Theory, Algorithms, and Applications. Prentice Hall, Englewood Cliffs, NJ, 1993.

[2] R. K. Ahuja, K. Mehlhorn, J. B. Orlin, and R. E. Tarjan. Faster algorithms for the shortest path problem. Journal of the ACM, 37:213-233, 1990.

[3] R. K. Ahuja, J. B. Orlin, and R. E. Tarjan. Improved time bounds for the maximum flow problem. SIAM Journal on Computing, 18:939-954, 1989.

[4] F. Baharona and É. Tardos. Note on Weintraub's minimum-cost circulation algorithm. SIAM Journal on Computing, 18:579-583, 1989.

[5] R. E. Bellman. On a routing problem. Quart. Appl. Math., 16:87-90, 1958.

[6] R. G. Bland. New finite pioting rules for the simplex method. Mathematics of Operations Research, 2:103-107, 1977.

[7] R. G. Busacker and P. J. Gowen. A procedure for determining a family of minimum-cost network flow patterns. Technical Report 15, Operations Research Office, Johns Hopkins University, 1961.

[8] R. G. Busacker and T. L. Saaty. Finite graphs and networks: an introduction with applications. McGraw-Hill, New York, 1965.

[9] V. Chvatal. Linear programming. W. H. Freeman, New York, 1983.

[10] E. Cohen and N. Megiddo. New algorithms for generalized network flows. Mathematical Programming, 64:325-336, 1994.

[11] G. B. Dantzig. Applications of the simplex method to a transportation problem. In T. C. Koopmans, editor, Activity analysis and production and allocation, pages 359-373. Wiley, New York, 1951. 
[12] G. B. Dantzig. Linear programming and extensions. Princeton University Press, Princeton, NJ, 1962.

[13] J. Edmonds and R. M. Karp. Theoretical improvements in algorithmic efficiency for network flow problems. Journal of the ACM, 19:248-264, 1972.

[14] J. Elam, F. Glover, and D. Klingman. A strongly convergent primal simplex algorithm for generalized networks. Mathematics of Operations Research, 4:39 59, 1979 .

[15] L. R. Ford and D. R. Fulkerson. Maximal flow through a network. Canadian Journal of Mathematics, 8:399-404, 1956.

[16] L. R. Ford and D. R. Fulkerson. Flows in Networks. Princeton University Press, Princeton, NJ, 1962.

[17] M. L. Fredman and R. E. Tarjan. Fibonacci heaps and their uses in improved network optimization algorithms. Journal of the ACM, 34:596-615, 1987.

[18] D. R. Fulkerson. An out-of-kilter method for minimal cost flow problems. SIAM Journal, 9:13-27, 1961.

[19] F. Glover and D. Klingman. On the equivalence of some generalized network flow problems to pure network problems. Mathematical Programming, 4:269 278,1973

[20] A. V. Goldberg, S. A. Plotkin, and É. Tardos. Combinatorial algorithms for the generalized circulation problem. Mathematics of Operations Research, 16:351379, 1991.

[21] A. V. Goldberg and S. Rao. Length functions for flow computations. Technical Report 97-055, NEC Research Institute, 1997.

[22] A. V. Goldberg and R. E. Tarjan. A new approach to the maximum flow problem. Journal of the ACM, 35:921-940, 1988.

[23] A. V. Goldberg and R. E. Tarjan. Finding minimum-cost circulations by canceling negative cycles. Journal of the ACM, 36:388-397, 1989.

[24] A. V. Goldberg and R. E. Tarjan. Solving minimum cost flow problems by successive approximation. Mathematics of Operations Research, 15:430-466, 1990. 
[25] D. Goldfarb and Z. Jin. A polynomial dual simplex algorithm for the generalized circulation problem. Technical report, Department of Industrial Engineering and Operations Research, Columbia University, 1995.

[26] D. Goldfarb and Z. Jin. A faster combinatorial algorithm for the generalized circulation problem. Mathematics of Operations Research, 21:529-539, 1996.

[27] D. Goldfarb, Z. Jin, and Y. Lin. A polynomial dual simplex algorithm for the generalized circulation problem. Technical report, Department of Industrial Engineering and Operations Research, Columbia University, 1998.

[28] D. Goldfarb, Z. Jin, and J. B. Orlin. Polynomial-time highest gain augmenting path algorithms for the generalized circulation problem. Mathematics of Operations Research, 22:793-802, 1997.

[29] M. Gondran and M. Minoux. Graphs and Algorithms. Wiley, New York, 1984.

[30] M. Iri. A new method of solving transportation-network problems. Journal of the Operations Research Society of Japan, 3:27-87, 1960.

[31] J. J. Jarvis and A. M. Jezior. Maximal flow with gains through a special network. Operations Research, 20:678-688, 1972.

[32] W. S. Jewell. Optimal flow through networks. Technical Report 8, Operations Research Center, MIT, 1958.

[33] W. S. Jewell. Optimal flow through networks with gains. Operations Research, 10:476-499, 1962.

[34] D. B. Johnson. A priority queue in which initialization and queue operations take $O(\log \log d)$ time. Mathematical Systems Theory, 14:295-309, 1982.

[35] A. Kamath and O. Palmon. Improved interior point algorithms for exact and approximate solution of multicommodity flow problems. In Proceedings of the 6th Annual ACM-SIAM Symposium on Discrete Algorithms, pages 502-511, 1995.

[36] S. Kapoor and P. M. Vaidya. Speeding up Karmarkar's algorithm for multicommodity flows. Mathematical Programming, 73:111-127, 1997.

[37] N. Karmarkar. A new polynomial-time algorithm for linear programming. Combinatorica, 4:373-395, 1984. 
[38] R. M. Karp. A characterization of the minimum cycle mean in a digraph. Discrete Mathematics, 23:309-311, 1978.

[39] V. King, S. Rao, and R. E. Tarjan. A faster deterministic maximum flow algorithm. Journal of Algorithms, 17:447-474, 1994.

[40] M. Klein. A primal method for minimal cost flows with applications to the assignment and and transportation problems. Management Science, 14:205$220,1967$.

[41] J.K. Lenstra, D. B. Shmoys, and É. Tardos. Approximation algorithms for scheduling unrelated parallel machines. Mathematical Programming, 46:259 $271,1990$.

[42] S. M. Murray. An interior point approach to the generalized flow problem with costs and related problems. PhD thesis, Stanford University, 1993.

[43] J. D. Oldham. Combinatorial approximation algorithms for generalized flow problems. Submitted to SODA, 1998.

[44] K. Onaga. Dynamic programming of optimum flows in lossy communication nets. IEEE Trans. Circuit Theory, 13:308-327, 1966.

[45] J. B Orlin. A faster strongly polynomial minimum cost flow algorithm. Operations Research, 41:338-350, 1993.

[46] J. B. Orlin and R. K. Ahuja. New scaling algorithms for the assignment and minimum cycle mean problems. Mathematical Programming, 54:41-56, 1992.

[47] S. Phillips and J. Westbrook. Online load balancing and network flow. In Proceedings of the 25th Annual ACM Symposium on Theory of Computing, pages 402-411, 1993.

[48] T. Radzik. Approximate generalized circulation. Technical Report 93-2, Cornell Computational Optimization Project, Cornell University, 1993.

[49] T. Radzik. Faster algorithms for the generalized network flow problem. Mathematics of Operations Research, 23:69-100, 1998.

[50] J. Renegar. A polynomial time algorithm, based on newton's method, for linear programming. Mathematical Programming, 40:59-94, 1988.

[51] D. D. Sleator and R. E. Tarjan. A data structure for dynamic trees. Journal of Computer and System Sciences, 26:362-391, 1983. 
[52] É. Tardos. Personal communication, 1998.

[53] M. Thorup. Undirected single source shortest paths in linear time. In 38th Annual IEEE Symposium on Foundations of Computer Science, pages 12-21, 1997.

[54] K. Truemper. Optimal flows in networks with positive gains. PhD thesis, Case Western Reserve University, 1973.

[55] K. Truemper. On max flows with gains and pure min-cost flows. SIAM Journal on Applied Mathematics, 32:450-456, 1977.

[56] P. Tseng and D. P. Bertsekas. An $\epsilon$-relaxation method for separable convex cost generalized network flow problems. In 5th International Integer Programming and Combinatorial Optimization Conference, 1996.

[57] P. M. Vaidya. Speeding up linear programming using fast matrix multiplication. In 30th Annual IEEE Symposium on Foundations of Computer Science, pages 332-337, 1989.

[58] K. D. Wayne. A polynomial-time combinatorial algorithm for generalized minimum cost flow. In preparation, 1998.

[59] K. D. Wayne and L. Fleischer. Faster approximation algorithms for generalized flow. Submitted to SODA, 1998. 